University of Tennessee Health Science Center

UTHSC Digital Commons

\title{
Evaluating the Therapeutic Effect of an Hsp90 Inhibitor in Mouse Models of Alzheimer's Disease
}

\author{
Bin Wang \\ University of Tennessee Health Science Center
}

Follow this and additional works at: https://dc.uthsc.edu/dissertations

Part of the Neurosciences Commons

\section{Recommended Citation}

Wang, Bin (http://orcid.org/0000-0001-9032-5009), "Evaluating the Therapeutic Effect of an Hsp90 Inhibitor in Mouse Models of Alzheimer's Disease" (2016). Theses and Dissertations (ETD). Paper 402. http://dx.doi.org/10.21007/etd.cghs.2016.0412. 


\title{
Evaluating the Therapeutic Effect of an Hsp90 Inhibitor in Mouse Models of Alzheimer's Disease
}

\begin{abstract}
The excessive accumulation of amyloid peptides $(A \beta)$ represents one major pathological hallmark of Alzheimer's disease (AD), which is most notably characterized by synaptic dysfunction. Strategies targeting heat shock protein 90 (Hsp90) inhibition have been widely investigated in the treatment of cancer for over two decades. Its application in the treatment of neurodegenerative diseases however, has emerged more recently in the last decade. The role of the Hsp90 chaperone in clearing misfolded protein aggregates has been well established (in vitro only), but its function in synaptic activity remains elusive.
\end{abstract}

In our study, we utilized a widely used Hsp90 inhibitor, 17-AAG (17-allylamino-17-

desmethoxygeldanamycin), to show that 17-AAG not only induces a heat shock-like response,

but also regulates (likely at the transcriptional level as modeled by qRT-PCR) select proteins enriched in the synapse, such as Post-synaptic density 95 (PSD95), synapsin I, and brain-derived neurotrophic factor (BDNF), among others. Confocal imaging demonstrated rescued dendritic spines with 17-AAG treatment after $A \beta$ challenge in neuronal culture models. The functionality of these changes in the synapse was further confirmed by memory improvement in contextual fear conditioning tests. This work implied a potential strategy in using Hsp90 inhibitors to treat AD. Nevertheless, it must be noted that 17-AAG has limited feasibility in therapy due to its poor CNS-permeability.

In order to test the hypothesis inspired and encouraged by the initial 17-AAG experimental results, we instead used a similar compound with favorable CNS-permeability. OS47720, a radicicol derivative Hsp90 inhibitor, produced comparable effects to those of 17- AAG in inducing heat shock-like responses and promoting synaptic protein expression. Electrophysiology studies on coherence demonstrated enhancement of neuronal activity within the cortical hippocampal network. Most importantly, chronic 0S47720 administration for three months and six months in early and middle symptomatic AD mouse model (Tg2576) both rescued memory deficits without obvious systemic toxicity. OS47720, as an Hsp90 inhibitor, was the first reported compound to display therapeutic effects after long-term treatment in an $A D$ animal model. Post-mortem studies revealed elevation in protein expression of heat shock proteins, synaptic proteins, and BDNF following treatment, without affecting $A \beta$ load and neuroinflammation. Downregulation of heat shock factor 1 (HSF1) by either a pharmacological inhibitor or a virus-delivered shRNA abolished OS47720's effects on memory improvement against A $\beta$ toxicity. Overexpressing HSF1 partially improved memory. Together with the notion that HSF1-regulated BDNF mRNA, this implicated an HSF1-mediated mechanism at the transcriptional level of synaptic genes. Importantly, these observable effects bypassed several important signaling pathways, including the survival Protein kinase B (PKB or AKT) pathway which is an underlying mechanism of most neuroprotective drugs.

These findings revealed a novel function of $\mathrm{Hsp} 90$ inhibition in regulating synaptic gene expression and synaptic activity, further supporting the potential of using $\mathrm{Hsp} 90$ inhibitors in treating neurodegenerative diseases such as AD.

\section{Document Type}

Dissertation

Degree Name

Doctor of Philosophy (PhD) 


\section{Program}

Biomedical Sciences

Research Advisor

Francesca-Fang Liao, Ph.D.

\section{Keywords}

Alzheimer's disease, Heat shock inhibitor, Synapse protection

\section{Subject Categories}

Medical Sciences | Medicine and Health Sciences | Neurosciences 


\title{
Evaluating the Therapeutic Effect of an Hsp90 Inhibitor in Mouse Models of
} Alzheimer's Disease

\author{
A Dissertation \\ Presented for \\ The Graduate Studies Council \\ The University of Tennessee \\ Health Science Center \\ In Partial Fulfillment \\ Of the Requirements for the Degree \\ Doctor of Philosophy \\ From The University of Tennessee
}

\author{
By \\ Bin Wang \\ August 2016
}


Copyright (C) 2016 by Bin Wang. All rights reserved. 


\section{DEDICATION}

This dissertation is dedicated to my parents, Qianghua Wang and Xiaoqin Yang, who have demonstrated the utmost support and encouragement throughout my continual pursuit of knowledge. I also dedicate this dissertation to my dear wife, Xudong Huang, and my lovely daughter, Ashley Wang for their love and support. 


\section{ACKNOWLEDGEMENTS}

Firstly, I would like to express my special appreciation and thanks to my mentor Dr. Francesca-Fang Liao for her continuous and tremendous support of my $\mathrm{Ph}$.D studies and related research. Her guidance, encouragement, and mentorship were invaluable throughout my studies.

I would also like to thank the graduate committee of Prof. Rennolds S. Ostrom, Prof. Williams E. Armstrong, Prof. Melloni Cook, Prof. Michael P. McDonald, and Prof. Kazuko Sakata not only for their insightful comments and encouragements, but also for challenging me to widen the scope of my research.

I sincerely thank my fellow labmates in Dr. Liao's lab for their stimulating discussions, and for all the fun we have had in the past years. In particular, I am grateful to Dr. Yaomin Chen for enlightening me in the formulation stage of the research project. Additionally, my thanks also go to Mr. Raymond Xu, Dr. Kyle Summers and Dr. Jingjing Li for their suggestions on the thesis writing.

My thanks also goes to The Neuroscience track in Integrated Program for Biomedical Science (IPBS), whom have provided an exemplary training environment. Last but not least, I would like to thank my friends and my family - my wife Xudong Huang and my parents for their support, and my daughter Ashley Wang for bringing happiness throughout my life. 


\begin{abstract}
The excessive accumulation of amyloid peptides $(A \beta)$ represents one major pathological hallmark of Alzheimer's disease (AD), which is most notably characterized by synaptic dysfunction. Strategies targeting heat shock protein 90 (Hsp90) inhibition have been widely investigated in the treatment of cancer for over two decades. Its application in the treatment of neurodegenerative diseases however, has emerged more recently in the last decade. The role of the Hsp90 chaperone in clearing misfolded protein aggregates has been well established (in vitro only), but its function in synaptic activity remains elusive.

In our study, we utilized a widely used Hsp90 inhibitor, 17-AAG (17-allylamino17-desmethoxygeldanamycin), to show that 17-AAG not only induces a heat shock-like response, but also regulates (likely at the transcriptional level as modeled by qRT-PCR) select proteins enriched in the synapse, such as Post-synaptic density 95 (PSD95), synapsin I, and brain-derived neurotrophic factor (BDNF), among others. Confocal imaging demonstrated rescued dendritic spines with 17-AAG treatment after A $\beta$ challenge in neuronal culture models. The functionality of these changes in the synapse was further confirmed by memory improvement in contextual fear conditioning tests. This work implied a potential strategy in using Hsp90 inhibitors to treat AD. Nevertheless, it must be noted that 17-AAG has limited feasibility in therapy due to its poor CNS-permeability.
\end{abstract}

In order to test the hypothesis inspired and encouraged by the initial 17-AAG experimental results, we instead used a similar compound with favorable CNSpermeability. OS47720, a radicicol derivative Hsp90 inhibitor, produced comparable effects to those of 17-AAG in inducing heat shock-like responses and promoting synaptic protein expression. Electrophysiology studies on coherence demonstrated enhancement of neuronal activity within the cortical hippocampal network. Most importantly, chronic 0S47720 administration for three months and six months in early and middle symptomatic AD mouse model (Tg2576) both rescued memory deficits without obvious systemic toxicity. OS47720, as an Hsp90 inhibitor, was the first reported compound to display therapeutic effects after long-term treatment in an AD animal model. Postmortem studies revealed elevation in protein expression of heat shock proteins, synaptic proteins, and BDNF following treatment, without affecting $\mathrm{A} \beta$ load and neuroinflammation. Downregulation of heat shock factor 1 (HSF1) by either a pharmacological inhibitor or a virus-delivered shRNA abolished OS47720's effects on memory improvement against A $\beta$ toxicity. Overexpressing HSF1 partially improved memory. Together with the notion that HSF1-regulated BDNF mRNA, this implicated an HSF1-mediated mechanism at the transcriptional level of synaptic genes. Importantly, these observable effects bypassed several important signaling pathways, including the survival Protein kinase B (PKB or AKT) pathway which is an underlying mechanism of most neuroprotective drugs. 
These findings revealed a novel function of Hsp90 inhibition in regulating synaptic gene expression and synaptic activity, further supporting the potential of using Hsp90 inhibitors in treating neurodegenerative diseases such as AD. 


\section{TABLE OF CONTENTS}

CHAPTER 1. LITERATURE REVIEW AND HYPOTHESIS .......................................1

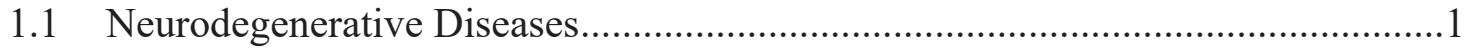

1.1.1 Neurodegenerative Diseases.................................................................

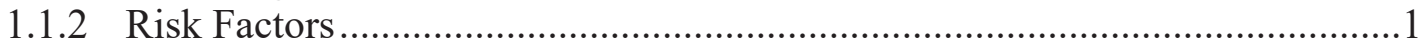

1.1.3 Protein Misfolding and Aggregation in Neurodegeneration ..........................

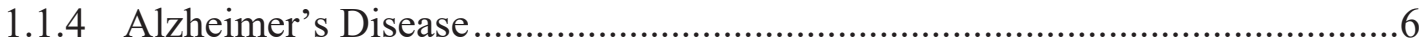

1.1.5 Alzheimer's Disease: Synapse Disease ....................................................

1.1.6 Mouse Models of Alzheimer's Disease .........................................................

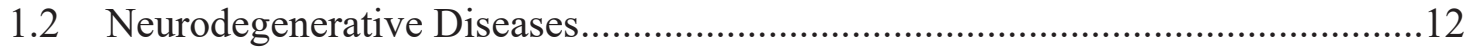

1.2.1 Heat Shock Response and Heat Shock Proteins...........................................12

1.2.2 Hsp90 and Hsp70: Structure-based Distinct Functions..................................15

1.2.3 Heat Shock Proteins in Neurodegeneration...............................................19

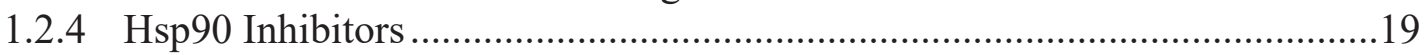

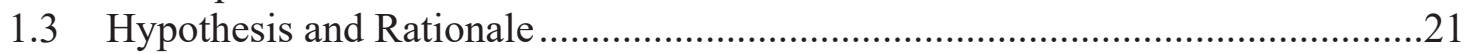

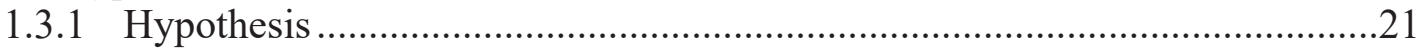

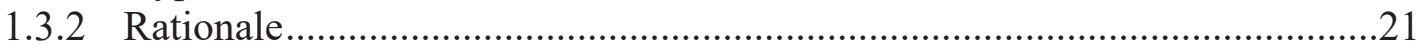

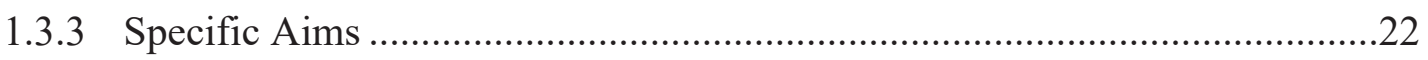

CHAPTER 2. MATERIALS AND METHODS......................................................23

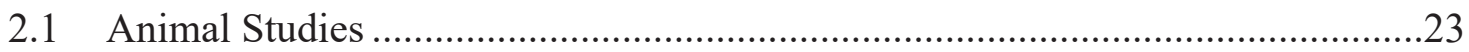

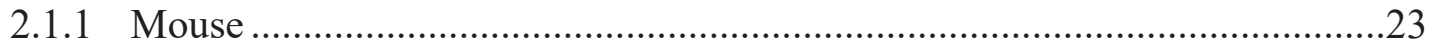

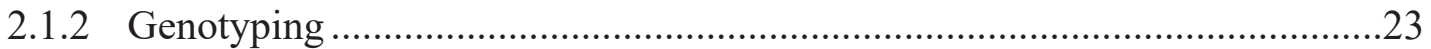

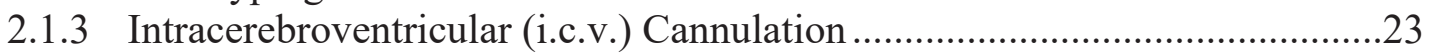

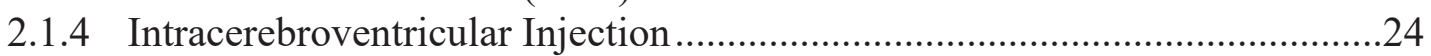

2.1.5 Intra-hippocampal CA1 Viral Microinjection ............................................24

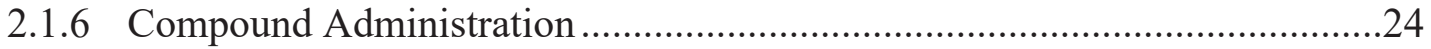

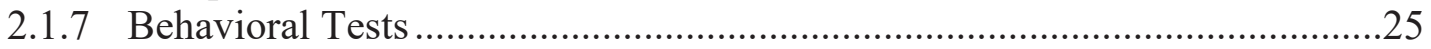

2.1.7.1 Contextual Fear Conditioning .......................................................... 25

2.1.7.2 Open Field Test ............................................................................. 25

2.1.7.3 Open-field Locomotor Activity ..................................................... 25

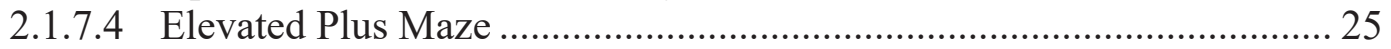

2.1.7.5 Novel Object Recognition ............................................................ 26

2.1.7.6 Cross Maze ......................................................................................... 26

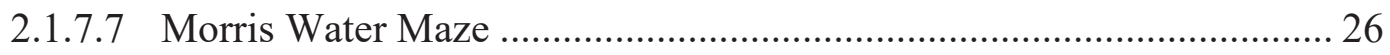

2.1.8 Perfusion and Fixation for Histology ........................................................26

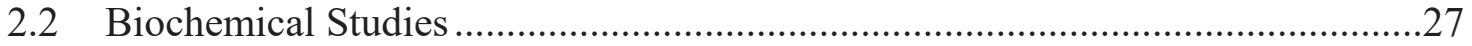

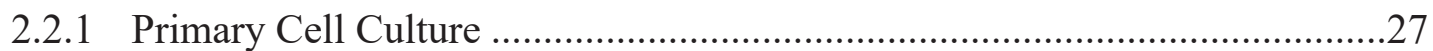

2.2.2 Immunocytochemistry and Dendritic Spine Quantification.........................27

2.2.3 Protein Analysis ...........................................................................................28

2.2.4 Naturally Aecreted A $\beta$-containing Conditioned Medium .............................28

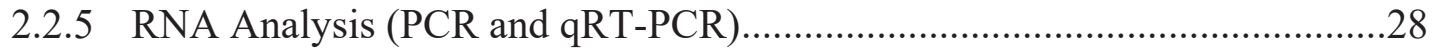

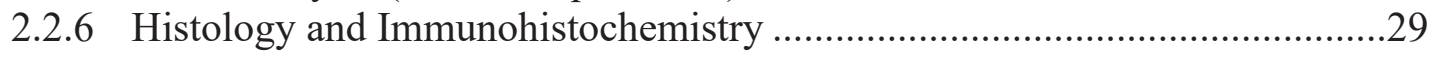

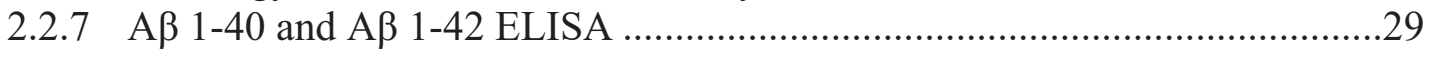


2.2.8 Liquid Chromatography-mass Spectrometry (LC-MS) .................................29

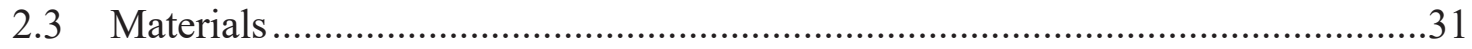

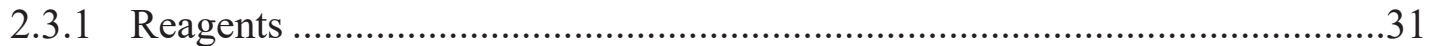

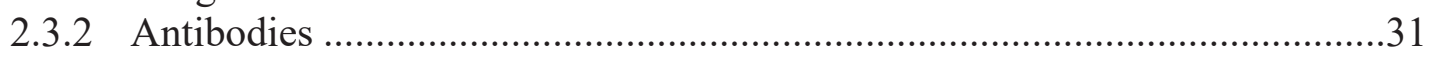

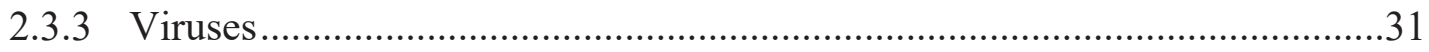

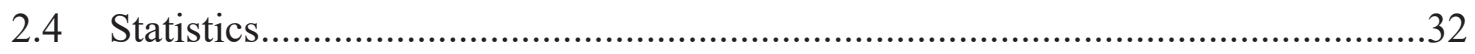

CHAPTER 3. 17-AAG'S NEUROPROTECTION AGAINST A $\beta$ TOXICITY .........33

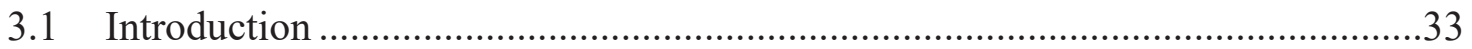

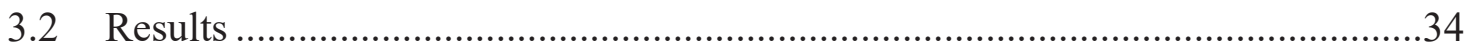

3.2.1 17-AAG Induces Rapid and Sustained Expression of HSPs and Synaptic

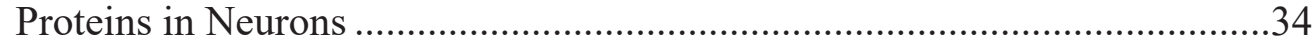

3.2.2 17-AAG Protects Synaptic Structure Against A $\beta$..........................................36

3.2.3 17-AAG Transcriptionally Upregulates Synaptic Proteins ................................36

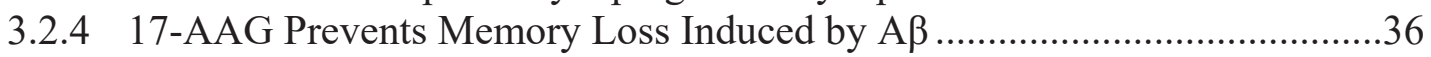

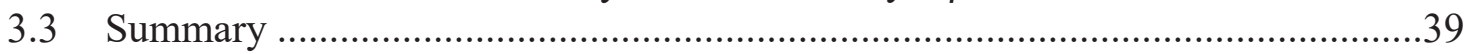

\section{CHAPTER 4. EVALUATION OF THERAPEUTIC EFFECT OF OS47720 ............41}

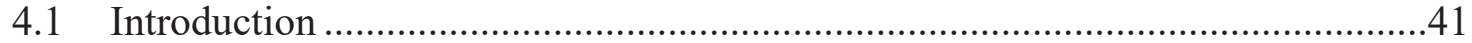

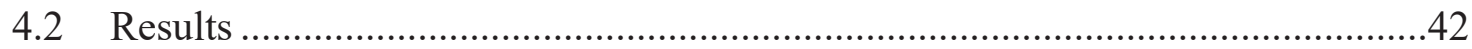

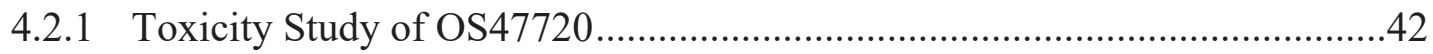

4.2.2 OS47720 Induces HSPs and Synaptic Proteins in Neurons ............................44

4.2.3 OS47720 Protects Synaptic Structures Against A $\beta$........................................44

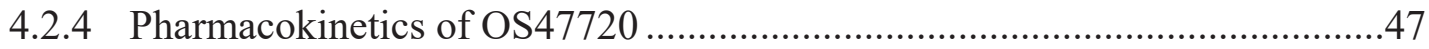

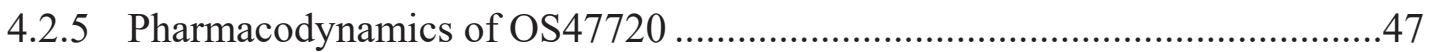

4.2.6 OS47720 Rescues Contextual Conditioned Freezing Memory Loss

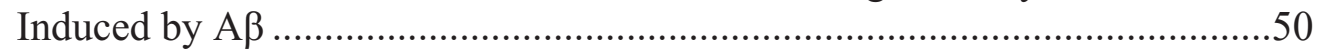

4.2.7 Chronic OS47720 Treatment Did Not Change Either Anxiety or Locomotor Activity .................................................................................50

4.2.8 OS47720 Rescues Working Memory and Spatial and Learning Memory Deficits in Presymptomatic Tg2576 Mice....................................................53

4.2.9 Protein Changes in Presymptomatic Tg2576 Mice after OS47720

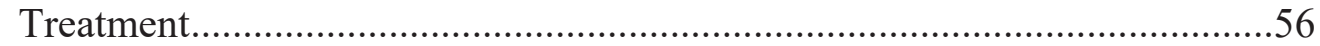

4.2.10 OS47720 Rescues Working Memory and Spatial and Learning Memory Deficits in Symptomatic Tg2576 Mice .......................................................56

4.2.11 End Point Study Revealed Protein Changes in Symptomatic Tg2576

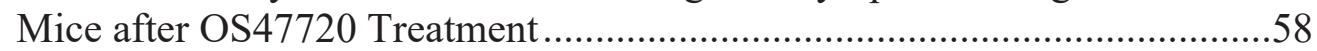

4.2.12 Body Weight Changes in Presymptomatic and Symptomatic Tg2576 Mice During OS47720 Treatment............................................................58

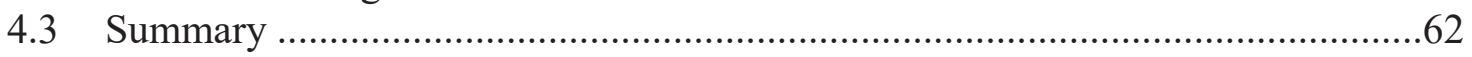

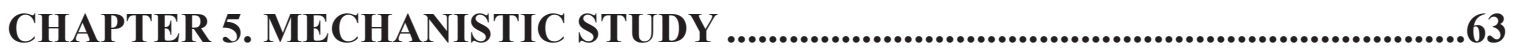

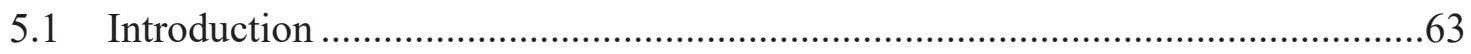

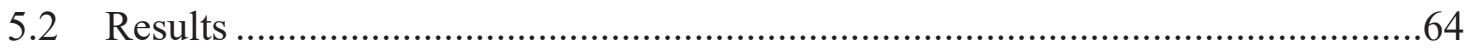

5.2.1 OS47720 Did Not Affect A $\beta$ Level and Neuroinflammation .........................64 
5.2.2 KRIBB11 Abolished Heat Shock-like Response and Contextual Conditioned Freezing Memory ………………………..................................64

5.2.3 Upregulation and Downregulation of HSF1 by Viruses .................................66

5.2.4 Downregulation of HSF 1 by AAVshRNAhsfl Abolished the Effect of

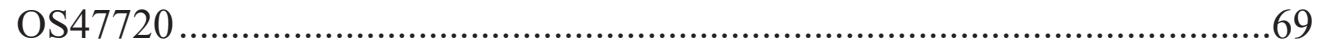

5.2.5 HSF1 Heterozygous Mice Exhibited Impaired Memory ………………….....69

5.2.6 Overexpression of HSF1 by AAVH-BH Slightly Improved Contextual Conditioned Freezing Memory ……….........................................................72

5.2.7 HSF 1 Is Required for the Initial Stage of Memory Consolidation and

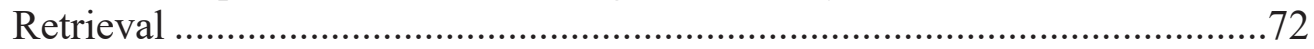

5.2.8 HSF1 Overexpression Induced BDNF Expression .......................................72

5.2.9 OS47720's Effect Bypasses cAMP/PKA and PI3K/AKT Pathways but

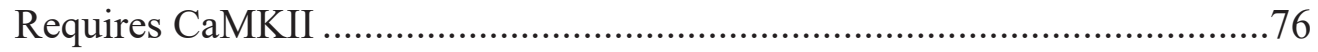

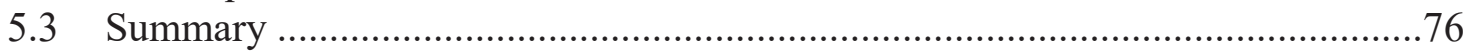

CHAPTER 6. DISCUSSION, CONCLUSIONS AND FUTURE DIRECTIONS......78

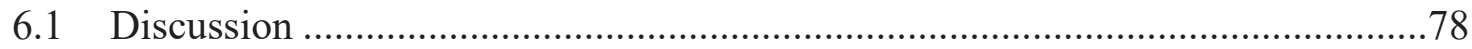

6.1.1 Investigation of Hsp90 Inhibitors in Neurodegenerative Diseases .................78

6.1.2 Heat Shock Response in Neuronal Cells ......................................................81

6.1.3 Novel Findings: HSF1-mediated Mechanism on Synapses ............................82

6.1.4 Transcriptional Regulation by HSF 1: Potentially Another Master

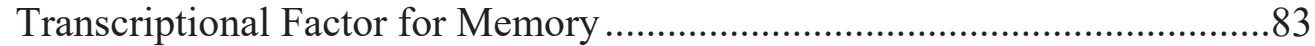

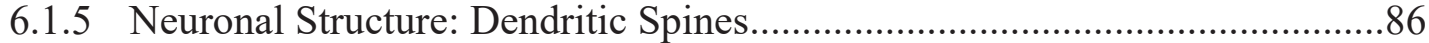

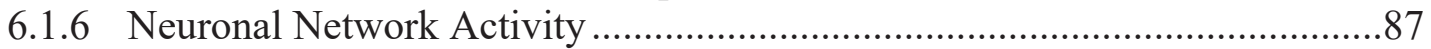

6.1.7 Impaired HSF1 and Dysregulation in Neurodegeneration .............................8

6.1.8 Therapeutic Implication of OS47720 in AD .............................................89

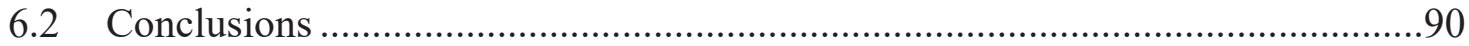

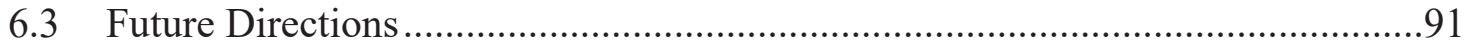

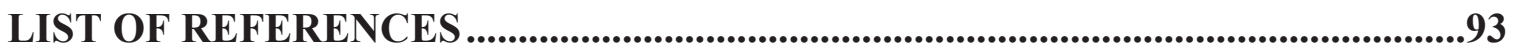

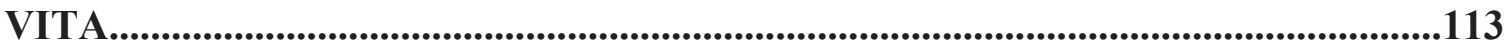




\section{LIST OF TABLES}

Table 1-1 Disease Proteins and Pathology in Major Neurodegenerative Disease. .........7

Table 1-2 Generations of Commonly Used Mouse Models of AD..............................13

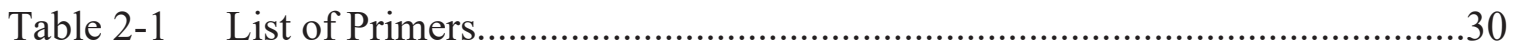

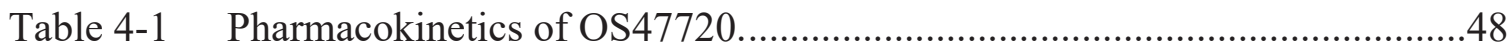

Table 6-1 Summary of the In vivo Data Using Hsp90 Inhibitors in Neurodegeneration in Mouse Models. .80 


\section{LIST OF FIGURES}

Figure 1-1 APP Processing and A $\beta$ Accumulation. ………………….........................

Figure 1-2 HSF1-mediated Heat Shock Response. .......................................................16

Figure 1-3 Structure and Functions of Hsp90 Dimer. .......................................................18

Figure 3-1 17-AAG Induces HSF1 Nuclear Translocation, HSPs and Synaptic

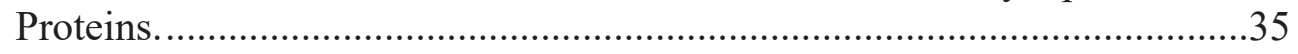

Figure 3-2 17-AAG Protects Neurons from A $\beta$-induced Dendritic Spine Loss..............37

Figure 3-3 17-AAG Activates Transcription of Select Synaptic Genes...........................38

Figure 3-4 17-AAG Prevents Memory Loss Induced by $\mathrm{A} \beta$ In vivo..................................40

Figure 4-1 Compounds' Structures and Toxicity .......................................................43

Figure 4-2 Nuclear Translocation of HSF1 Induced by OS47720 in Culture Mature

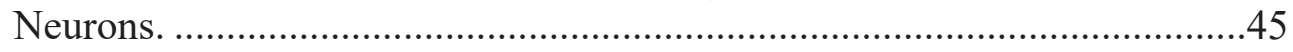

Figure 4-3 OS47720 Exhibits Synaptic Protection Against A $\beta$ Toxicity........................46

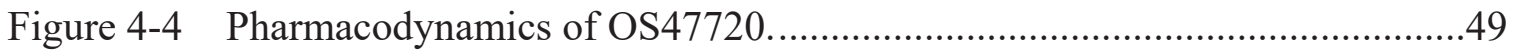

Figure 4-5 OS47720 Induces Hsp70 Protein Expression on Reporter Mice...................51

Figure 4-6 OS47720 Prevents Memory Loss Induced by A $\beta$ In vivo. .............................52

Figure 4-7 Locomotor Activity and Anxiety Level after 3-month Treatment of

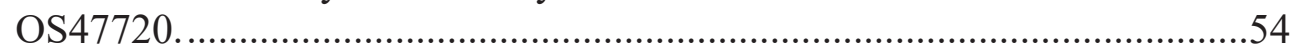

Figure 4-8 In vivo Therapeutic Efficacy of OS47720 in Tg2576 Mice..........................55

Figure 4-9 End Point Western Blot Analysis after 3-month Treatment of OS47720.....57

Figure 4-10 Effects on Symptomatic Tg2576 Mice after 6-month Treatment of OS47720.

Figure 4-11 End Point Western Blot Analysis after 6-month Treatment of OS47720.....60

Figure 4-12 Body Weights from Two Cohorts of Tg2576 Mice with Chronic Treatment of OS47720.

Figure 5-1 OS47720 Treatment Has No Effect on $\mathrm{A} \beta$ and Plaque Load in 18 Month-old Tg2576 Mice. 
Figure 5-2 KRIBB11 Abolishes Contextual Fear Memory.

Figure 5-3 Upregulation and Downregulation of HSF1 by AAVH-BH and

AAVshRNAhsf1 in Cultured Primary Neurons.

Figure 5-4 AAVshRNAhsf1 Abolishes the Effect of OS47720 in Contextual Fear Conditioning.................................................................................. 70

Figure 5-5 Behavioral Tests on Young Adult HSF1+/- and Littermate Control Mice...71

Figure 5-6 Overexpression of H-BH Did Not Exhibit Comparable Neuroprotection to OS47720 in Mice.

Figure 5-7 Time Course Study of KRIBB11 Treatment in Contextual Conditioned Freezing Memory Test. ............................................................... 74

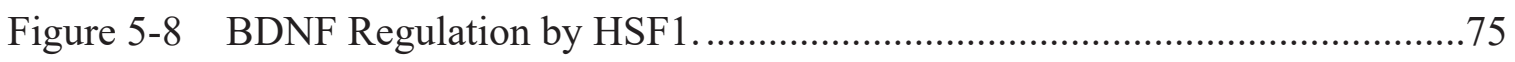

Figure 5-9 Hsp90 Inhibition Bypasses Several Major Signaling Pathways..................77

Figure 6-1 Schematic Illustration of HSF1-mediated Mechanism. .............................84 


\section{LIST OF ABBREVIATIONS}

\begin{tabular}{|c|c|}
\hline${ }^{\circ} \mathrm{C}$ & Celsius degrees \\
\hline$\mu \mathrm{g}$ & microgram \\
\hline$\mu 1$ & microliter \\
\hline$\mu 1$ & micrometer \\
\hline$\mu \mathrm{M}$ & micromolar \\
\hline 17-AAG & 17-allylamino-17-desmethoxygeldanamycin \\
\hline 17-DMAG & 17-(2-dimethylaminoethyl)-amino-17-desmethoxygeldanamycin \\
\hline $\mathrm{AD}$ & Alzheimer's disease \\
\hline ALS & Amyotrophic Lateral Sclerosis \\
\hline APP & Amyloid precursor protein \\
\hline ATP & Adenosine triphosphate \\
\hline $\mathrm{A} \beta$ & $\beta$-amyloid \\
\hline $\mathrm{BBB}$ & Blood-brain barrier \\
\hline $\mathrm{BDNF}$ & Brain-derived neurotrophic factor \\
\hline CBD & Corticobasal degeneration \\
\hline DLB & Dementia with Lewy Bodies \\
\hline DMSO & Dimethyl sulfoxide \\
\hline EDTA & Ethylenediaminetetraacetic acid \\
\hline FAD & Familial Alzheimer's disease \\
\hline FTD & Frontotemporal dementia \\
\hline g & gram \\
\hline GA & Geldanamycin \\
\hline
\end{tabular}




\begin{tabular}{|c|c|}
\hline GFAP & Glial fibrillary acidic protein \\
\hline $\mathrm{h}$ & hours \\
\hline HCIs & Hyaline conglomerate inclusions \\
\hline HD & Huntington's disease \\
\hline HRP & Horseradish peroxidase \\
\hline HSC70 & Heat shock cognate protein $70 \mathrm{kDa}$ \\
\hline HSE & Heat shock element \\
\hline HSF 1 & Heat shock factor 1 \\
\hline HSPs & Heat shock proteins \\
\hline Hsp40 & Heat shock protein 40 \\
\hline Hsp90 & Heat shock protein 27 \\
\hline Hsp90 & Heat shock protein 90 \\
\hline i.c.v. & Intracerebroventricular \\
\hline i.p. & Intraperitoneal \\
\hline iHsp70 & inducible heat shock protein $70 \mathrm{kDa}$ \\
\hline $\mathrm{kDa}$ & kilodalton \\
\hline $\mathrm{kg}$ & kilogram \\
\hline $\mathrm{L}$ & liter \\
\hline LBHIs & Lewy-body-like hyaline inclusions \\
\hline LC-MS & Liquid chromatography - mass spectrometry \\
\hline M & Molar \\
\hline $\mathrm{mg}$ & milligram \\
\hline $\min$ & minute \\
\hline
\end{tabular}




\begin{tabular}{|c|c|}
\hline $\mathrm{mL}$ & milliliter \\
\hline $\mathrm{mm}$ & millimeter \\
\hline $\mathrm{mM}$ & millimolar \\
\hline MSA & Multiple system atrophy \\
\hline n.d. & not determined \\
\hline ng & nanogram \\
\hline $\mathrm{nM}$ & nanomolar \\
\hline PBS & Phosphate-buffered saline \\
\hline PFA & Paraformaldehyde \\
\hline $\mathrm{PD}$ & Parkinson's disease \\
\hline $\mathrm{PD}$ & Pharmacodynamics \\
\hline PK & Pharmacokinetic \\
\hline PSD95 & Post-synaptic density 95 \\
\hline PSP & Progressive supranuclear palsy \\
\hline s & seconds \\
\hline SBMA & Spinobulbar muscular atrophy \\
\hline SCA & Spinocerebellar ataxia \\
\hline SDS & Sodium dodecyl sulfate \\
\hline SDS-PAGE & Sodium dodecyl sulfate - polyacrylamide gel electrophoresis \\
\hline SOD1 & Superoxide dismutase 1 \\
\hline TBS & Tris-buffered saline \\
\hline UPS & Ubiquitin-proteasome system \\
\hline
\end{tabular}




\section{CHAPTER 1. LITERATURE REVIEW AND HYPOTHESIS}

\subsection{Neurodegenerative Diseases}

\subsubsection{Neurodegenerative Diseases}

Neurodegeneration is an umbrella term describing neurodegenerative processes involving progressive loss of both structure and function in affected brain cells, which are primarily neurons. Neurodegenerative diseases are various types of brain diseases caused by neurodegeneration, the four most common of which include Alzheimer's disease (AD), Parkinson's disease (PD), Huntington's disease (HD), and Amyotrophic lateral sclerosis (ALS). Other less prevalent but similar diseases include Corticobasal degeneration (CBD), Progressive supranuclear palsy (PSP), Dementia with Lewy Bodies (DLB), Frontotemporal dementia (FTD), Frontotemporal dementia and parkinsonism linked to chromosome 17 (FTDP-17), Multiple system atrophy (MSA), Spinobulbar muscular atrophy (SBMA), and Spinocerebellar ataxia (SCA). Each of these diseases is believed to be irreversible and incurable, resulting in progressive deterioration of neuronal cells that eventually leads to death.

Neurodegeneration has two major types of consequences. The first is problematic mental functioning (dementia), while the other is the impaired movement (ataxia). Symptoms of each disease vary from person to person. Mental dysfunction is the most prominent symptom of $\mathrm{AD}$. Common symptoms of $\mathrm{AD}$ include progressive memory loss and confusion, difficulty in learning new things, problems with mood control, and trouble with language. PD, HD and ALS are also mainly characterized by movement problems. Symptoms of PD patients include slowed and limited movement, rigid and aching muscles (including throat muscles), and difficulty with walking and balance. HD patients experience memory loss, problems with decision-making and mood swings (depression, anger, etc.) in early stages. Later on, the following symptoms appear: involuntary twitching or other uncontrolled movements, rigid muscle, muscle contracture, difficulty in walking, abnormal eye movement, and difficulty with speaking and/or swallowing. PD and $\mathrm{HD}$ also demonstrate mental changes in the late stages of the disease that confer cognitive decline. ALS is mainly characterized as a movement disorder. ALS patients have progressive muscle weakness from disease onset, leading to unfortunate actions such as tripping, dropping things, and muscle cramps. When ALS worsens, the affected individual may lose voluntary control of basic actions such as laughing or crying, and more seriously may lose control of life-dependent actions such as breathing, necessitating permanent use of external ventillatory support (NINDS, 2013).

\subsubsection{Risk Factors}

Several risk factors have been linked to neurodegenerative diseases, the two most prominent of which are age and family history (genetic predisposition). Other risk factors 
include oxidative stress, inflammation, infection, stroke, hypertension, diabetes, head trauma, tumors, depression, smoking, vitamin deficiencies, race, gender, endocrine conditions, immune and metabolic conditions, chemical exposure and even level of education (Brown et al., 2005).

In $\mathrm{AD}$, age is the biggest risk factor. According to prevalence reports in 2013, AD prevalence rates were $0.28 \%$ in the population of $65-69$ years old. This number rose to $5.6 \%$ in individuals 90 years old and older population. About $4 \%$ of AD patients are less than 65 years old. $15 \%$ of patients are $65-74$ years old and $81 \%$ of patients are 75 years old or older (Hebert et al., 2013). Age is also the greatest risk factor in PD (Hindle et al., 2010). A meta-analysis showed an increasing prevalence of PD with age worldwide, from 107 per 100,000 patients in 50-59 years to 1903 per 100,000 patients in 80 years old and above (Pringsheim et al., 2014). Aging not only increases the probability of getting neurodegeneration, but also impairs neuronal self-repair. For instance, loss or restricted neurogenesis in the hippocampus, subventricular zone (SVZ)/rostral migratory stream (RMS) and olfactory epithelium (OE) was observed in the aging brain (Riddle and Lichtenwalner, 2007; Shetty et al., 2011).

Genetic predisposition represents another main risk factor for many types of neurodegenerative diseases, such as early-onset familial AD (eFAD or FAD), late onset AD (LOAD), familial early-onset Parkinsonism, HD, and Familial ALS (FALS). Though eFAD accounts for only a small percent of all AD cases (less than $5 \%$ ), it typically appears at an earlier age (less than 65 years), featuring genetic autosomal dominant inheritance within affected families (Bertram and Tanzi, 2005). Currently three different genes with over 160 mutations have been found to cause eFAD. These genes include the amyloid precursor protein on chromosome 21 (APP), presenilin 1 (PSEN1) on chromosome 14, and presenilin 2 (PSEN2) on chromosome 1 (Zhang et al., 1998). Among them, PSEN1 is the most common mutated gene which accounts for most AD cases with early disease onset before 50 years old. In contrast, LOAD mainly affects people aged over 65 years old and represents the majority of all AD cases. The gene most associated with LOAD is apolipoprotein E (APOE), which is located on chromosome 19q13.2. Among the many variants of the APOE gene, alleles $\varepsilon 2, \varepsilon 3$ and $\varepsilon 4$ are the three most common polymorphisms found in all populations (Strittmatter et al., 1993; Schmechel et al., 1993). Though the impact of genetic factors contributing to overall prevalence of $\mathrm{PD}$ is less significant than in AD, there are still more than 5 genes that have been reported to cause familial early-onset Parkinsonism (such as SNCA or PARK1: $\alpha-$ synuclein; PRKN or PARK2: parkin; DJ1 or PARK7: DJ-1; PINK1 or PARK6: PTENinduced putative kinase I; LRRK2 or PARK8: leucine-rich repeat kinase 2, also known as dardarin) (Bertram et al., 2005). Familial ALS (FALS) comprises about 10\% of all ALS cases, but that is not indicative of the overall influence genetic factors play in ALS. Several genes have been implicated with ALS development, including SOD1, TDP-43, MAPT, and ALS2. In contrast to these diseases affected by aging and multiple genetic mutations, HD is found to be solely hereditary and affected by a single gene, huntingtin (HTT) (Kremer et al., 1994). 
In addition to age-related and genetic factors, there are also other influences that have been known to be associated with neurodegenerative diseases. For instance, oxidative stress is a well-studied phenomenon that leads to free radical attack on neuronal cells. Imbalanced oxygen metabolism and excessive reactive oxygen species (ROS) in the brain are implicated in most neurodegenerative diseases (AD, PD, HD, ALS, etc.) (Uttara et al., 2009; Shukla et al., 2011). Neuroinflammation induced by systemic infection and activation of the innate immune response in the brain is mainly mediated by astrocyte and microglia, the key components of innate immune cells in the central nervous system (CNS). Increased neuroinflammation triggers neurotoxic pathways that cause progressive degeneration of neuronal cells (Perry et al., 2003; Amor et al., 2010; Amor et al., 2014). Besides these two most studied factors, there are also other contributing factors. A metaanalysis shows HD prevalence varied by races (Pringsheim et al., 2012) indicating existence of race factor. All these factors together with other potential or unknown factors lead to the occurrence of neurodegenerative diseases.

\subsubsection{Protein Misfolding and Aggregation in Neurodegeneration}

Though each disease has its own set of symptoms and associations with different gene mutations, there exists a common molecular pathway that has been implicated in various neurodegenerative diseases, which includes protein misfolding, accumulation and aggregation. It has been well established that accumulation and aggregation of misfolded proteins leads to synaptic alternation, neuronal damage, cell death, brain dysfunction, and disease (Soto and Estrada, 2008).

There are two histopathological hallmarks of AD: extracellularly localized senile plaques (also known as Alzheimer's plaques, neuritic plaques or amyloid plaques) and intracellularly localized neurofibrillary tangles (NFT), affecting brain regions including the hippocampus, cortex, basal forebrain, brain stem, and cerebellum. AD has been recognized as a protein misfolding disease because of the abnormal accumulation of misfolded Amyloid-beta $(\mathrm{A} \beta)$ proteins. The 4-kDa $\mathrm{A} \beta$ peptide was firstly isolated from $\mathrm{AD}$ and Down's syndrome patients and was identified to be the main component of the amyloid plaques (Glenner and Wong, 1984). The A $\beta$ is generated from its peptide precursor, Amyloid Precursor Protein (APP). APP has a transmembrane fragment structure that can be cleaved at the N-terminal domain of $A \beta$ by a membrane-associated beta-site amyloid precursor protein cleaving enzyme 1 ( $\beta$-secretase) between Met671 and Asp672. This cleavage process generates a large secreted derivative (soluble APP $\beta$, sAPP $\beta$ ) and a membrane-associated C-terminus fragment C99 (also known as CTF $\beta$ ). Additional cleavage of C99 by $\gamma$-secretase produces extracellular A $\beta$ species and the APP intracellular domain (AICD) (Gandy, 2005) (Figure 1-1). The PSEN1 protein coded by PSEN gene is the subunit of $\gamma$-secretase complex. Most extracellular A $\beta$ is composed of $A \beta 1-40$ (usually more than 95\%) and a minor proportion $A \beta 1-42$ (less than 5\%) (Naslund et al., 1994). The A $\beta$ monomer is generally harmless, but its physiological function is still not fully understood (Hiultunen et al., 2009). Several studies revealed potential functions of the $A \beta$ monomer that may include protection against oxidative stress, kinase enzyme activation, and proinflammatory activity (Baruch-Suchodolsky and Fischer., 2009; 


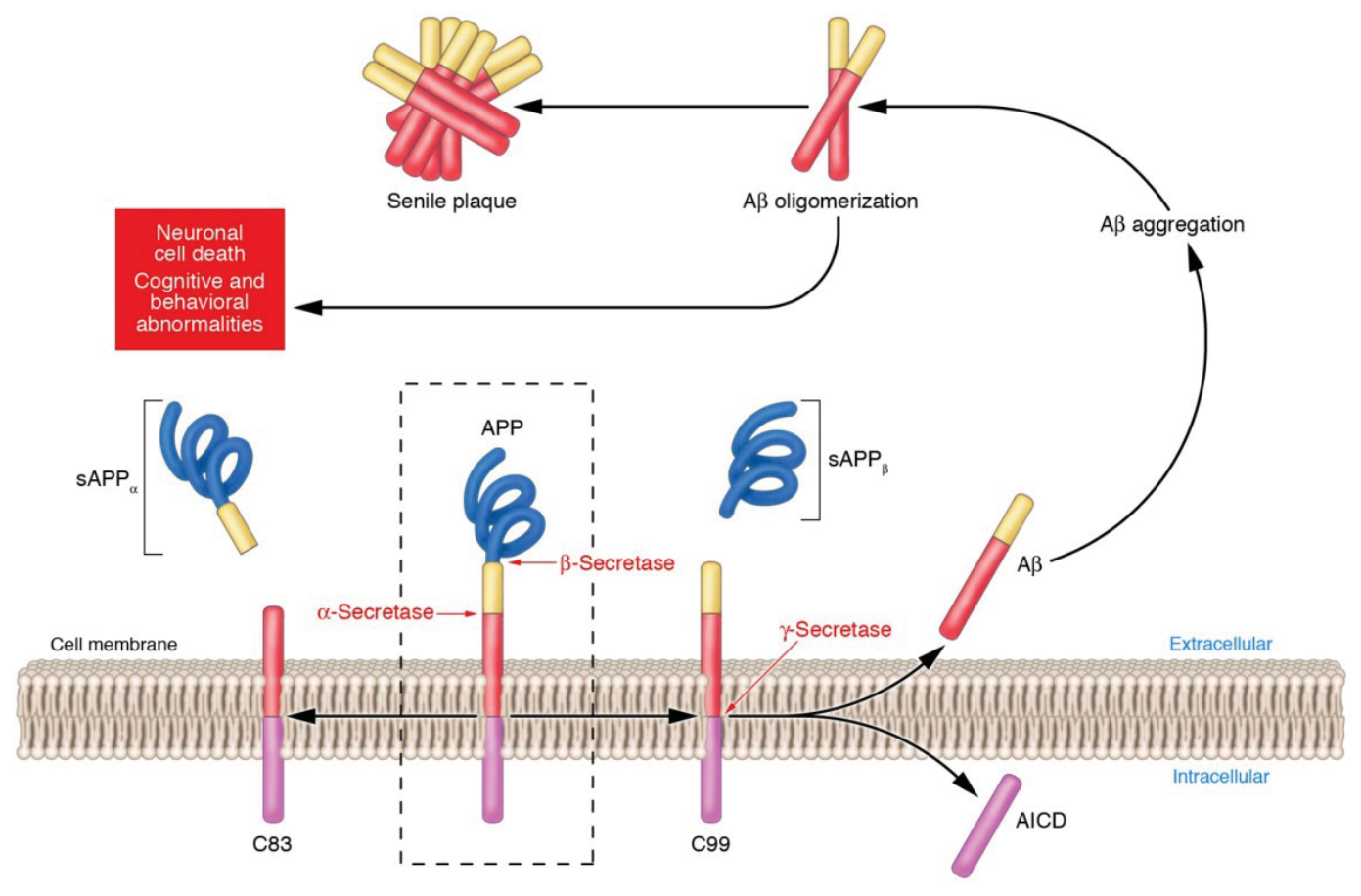

Figure 1-1 APP Processing and A $\beta$ Accumulation.

Reprinted with permission. Gandy S. (2005) The role of cerebral amyloid beta accumulation in common forms of Alzheimer disease. J Clin Invest. 115, 1121-1129. The cartoon illustrates the APP processing, A $\beta$ accumulation and formation of senile plaques. APP has a transmembrane fragment structure which can be cleaved at the Nterminal domain of $\mathrm{A} \beta$ by a membrane-associated beta-site amyloid precursor protein cleaving enzyme 1 ( $\beta$-secretase) between Met671 and Asp672. This cleavage process generates a large secreted derivative (soluble APP $\beta$, sAPP $\beta$ ) and a membrane-associated C-terminus fragment C99 (also known as CTF $\beta$ ). Additional cleavage of C99 by $\gamma$ secretase produces extracellular A $\beta$ species and APP intracellular domain (AICD). APP can be alternatively cleaved by $\alpha$-secretase producing extracellular secreted soluble APP $\alpha$ $(\operatorname{sAPP} \alpha)$ and a membrane-associated fragment C83. A $\beta$ aggregation and oligomerization cause synapse impairment, neuronal death, cognitive and behavioral abnormalities. Senile plaques are formed after considerable amount of $\mathrm{A} \beta$. 
Tabaton et al., 2010; Soscia et al., 2010). Some studies show participation of monomeric $\mathrm{A} \beta$ in modulating synaptic transmission, and that the $\mathrm{A} \beta$ monomer is required for synaptic plasticity and survival of neurons (Parihar and Brewer, 2010). Misfolding of the $\mathrm{A} \beta$ protein leads to $\mathrm{A} \beta$ aggregation, in which $\mathrm{A} \beta$ monomers aggregate to form dimers, trimers, tetramers, and oligomers. Eventually senile plaques are formed by further aggregation of A $\beta$ oligomerization. Alternatively, APP can be cleaved by $\alpha$-secretase between Lys687 and Leu688, resulting in generation of a membrane-bound fragment named C-terminus fragment $\mathrm{C} 83$ (also known as $\mathrm{CTF} \alpha$ ) and an extracellular fragment named soluble APP $\alpha(\mathrm{sAPP} \alpha)$, The extracellular fragment sAPP $\alpha$ was found as a nontoxic species and promotes growth of neurons before and after birth (Levites et al., 2003; Chasseigneaux et al., 2012). Notably, the intermediate misfolded A $\beta$ oligomers and aggregates, but not $A \beta$ monomers, small oligomers or mature polymers, are the most potent toxic species for all neuronal cells, promoting neurodegeneration by perturbing normal function of many subcellular organelles such as the mitochondria and endoplasmic reticulum (Mattson et al., 2001; Hashimoto et al., 2003; Pagani and Eckert, 2011). This series of events eventually causes neuronal cell death, and cognitive and behavioral abnormalities (Gandy, 2005; Sakano and Zako, 2010). AD is also recognized as a tauopathy due to the abnormally aggregated misfolded and hyperphosphorylated tau protein. Tau protein belongs to a group of cytoskeletal component proteins referred to as microtubule-associated proteins (MAP), which are abundantly expressed in neurons that bind to and stabilize microtubules. Conformational changes, hyperphosphorylation and misfoldings of tau lead to aberrant aggregations where paired helical filaments (PHF) of tau are formed, further aggregation leading to form the other hallmark of $\mathrm{AD}$, neurofibrillary tangles. The extent of the tau aggregation correlates with intensity of cell death and cognitive dysfunction (Iqbal et al., 2005). Tauopathy can also be seen in other neurodegenerative diseases such as Down's syndrome, Frontotemporal dementia (FTD), and Cortico-basal degeneration (CBD).

The pathological characteristics of PD are the progressive accumulation of misfolded $\alpha$-synuclein filaments and their aggregation in the form of Lewy bodies and Lewy neurites. A Lewy body is an inclusion body in the cytoplasm consisting of aggregated $\alpha$-synuclein protein and Lewy neurites contain granular material and abnormal $\alpha$-synuclein filaments (Dauer and Przedborski, 2003; Ross and Poirier, 2004). Misfolding and aggregation of $\alpha$-synuclein can be found in brain regions of diseased patients including the basal ganglia, sustantia nigra, and cortex.

HD is caused by a mutation in the HTT gene that abnormally expands an increased number of cytosine-adenine-guanine (CAG) nucleotide repeats, which leads to expression of the huntingtin (HTT) protein with an abnormally high number of glutamine (polyQ) repeats. The expansion of polyQ promotes misfolding and aggregation to form the disease-causing pathological hallmark of HD, intracellular aggregates of mutant $\mathrm{Htt}$ called inclusion bodies and intranuclear inclusions, which are usually located in the striatum, cortex, and basal ganglia regions. The formation of an inclusion body was found to be beneficial in HD by reducing levels of the toxic species of misfolded HTT protein from diffuse fraction. (The Huntington's Disease Collaborative Research Group, 1993; Arrasate and Finkbeiner, 2012). In familial amyotrophic lateral sclerosis (fALS), 
mutations in superoxide dismutase 1 (SOD1) are a causal factor to induce misfolding and aggregation of the SOD1 protein to form various types of inclusions in spinal motor neurons and the motor cortex (Prudencio and Borchelt, 2011). These inclusions include Lewy-body-like hyaline inclusions (LBHIs), hyaline conglomerate inclusions (HCIs), skein-like inclusions, Bunina bodies, and axonal spheroids (Kerman and Chakrabartty, 2011). These four major neurodegenerative diseases with disease-causing proteins are summarized in Table 1-1.

Protein misfolding and aggregation are also commonly found in other neurodegenerative diseases, such as misfolded and aggregated ataxin protein in spinocerebellar ataxia (SCA), misfolded androgen receptor (AR) in spinal bulbar muscular atrophy (SBMA), mutated/hyperphosphorylated tau misfolded protein in frontotemporal dementia (FTD), etc. This common molecular pathway has emerged as the mechanism of toxicity to neuronal cells and warrants further investigation into potential therapeutic approaches.

\subsubsection{Alzheimer's Disease}

Alzheimer's disease (AD) is currently the 6th leading overall cause of death overall, and the 5th leading cause of death for those over 65 years of age in the United States. It is the most common neurodegenerative disease, and is also the most common irreversible cause of dementia accounting for over $60 \%$ of all dementia cases (Wilson et al., 2012). Clinical signs of AD are mainly characterized by progressive deterioration and reduced activity of daily functioning abilities, along with neuropsychiatric symptoms and abnormal changes of behavior. In 2015, 5.3 million Americans were living with AD, a number that is projected to increase to 16 million by 2050. According to the Alzheimer's Association annual report, the direct cost burden of AD to American society to care for those who have AD is at USD \$226 billion for 2015, and according to one model, unless something is done, this cost will increase to $\$ 1.1$ trillion (in 2015 dollars). Unfortunately, there is no cure for $\mathrm{AD}$ and "AD is currently the only cause of death among the top 10 in the United States that cannot be prevented, cured, or even slowed" (Alzheimer's association, 2015).

$\mathrm{AD}$ affects people in different ways. The most common symptom in the initial stages is a gradual loss of ability to remember new things. This is because the first brain cells to degenerate are neurons localized in specific brain regions (for example hippocampus and cerebral cortex) that are involved in forming new memories. When degeneration spreads to neurons in other parts of the brain, individuals will experience more difficulties in daily life, including loss of memory, cognitive and language skills, and behavior changes. The brain will eventually shrink as a result of AD-induced tissue loss.

To date, there are five medications approved by the U.S. Food and Drug Administration (FDA) to treat the symptoms of AD. They are limited to acetylcholinesterase (AChE) inhibitors (donepezil, galantamine and rivastigmine) and $\mathrm{N}$ - 
Table 1-1 Disease Proteins and Pathology in Major Neurodegenerative Disease.

\begin{tabular}{|c|c|c|c|c|}
\hline Disease & $\begin{array}{l}\text { Associated } \\
\text { genes }\end{array}$ & $\begin{array}{l}\text { Major protein } \\
\text { deposited }\end{array}$ & $\begin{array}{c}\text { Characteristic } \\
\text { pathology }\end{array}$ & $\begin{array}{l}\text { Brain regions } \\
\text { affected }\end{array}$ \\
\hline $\begin{array}{l}\text { Alzheimer's } \\
\text { disease (AD) }\end{array}$ & $\begin{array}{l}\text { APP, PSEN1, } \\
\text { PSEN2, MAPT }\end{array}$ & $\begin{array}{l}\mathrm{A} \beta, \\
\text { hyperphospho- } \\
\text { rylated tau }\end{array}$ & $\begin{array}{l}\text { Neuritic } \\
\text { plaques and } \\
\text { neurofibrillary } \\
\text { tangles }\end{array}$ & $\begin{array}{l}\text { Hippocampus, } \\
\text { cortex, basal } \\
\text { forebrain, } \\
\text { brain stem, } \\
\text { cerebellum }\end{array}$ \\
\hline $\begin{array}{l}\text { Parkinson's } \\
\text { disease (PD) }\end{array}$ & $\begin{array}{l}\text { SNCA, DJ1, } \\
\text { PINK1, PARK, } \\
\text { LRRK2, etc. }\end{array}$ & $\alpha$-synuclein & $\begin{array}{l}\text { Lewy bodies } \\
\text { and Lewy } \\
\text { neurites }\end{array}$ & $\begin{array}{l}\text { Basal ganglia, } \\
\text { Sustantia } \\
\text { nigra, cortex }\end{array}$ \\
\hline $\begin{array}{l}\text { Huntington's } \\
\text { disease (HD) }\end{array}$ & HTT & Huntingtin & $\begin{array}{l}\text { Neuronal } \\
\text { inclusion body } \\
\text { and } \\
\text { Intranuclear } \\
\text { inclusions }\end{array}$ & $\begin{array}{l}\text { Striatum, other } \\
\text { basal ganglia, } \\
\text { cortex }\end{array}$ \\
\hline $\begin{array}{l}\text { Amyotrophic } \\
\text { lateral } \\
\text { sclerosis } \\
\text { (ALS) }\end{array}$ & $\begin{array}{l}\text { SOD1, TDP-43, } \\
\text { MAPT, etc }\end{array}$ & SOD1 & $\begin{array}{l}\text { Lewy-body- } \\
\text { like hyaline } \\
\text { inclusions, } \\
\text { Bonia bodies, } \\
\text { axonal } \\
\text { spheroids, etc. }\end{array}$ & $\begin{array}{l}\text { Spinal motor } \\
\text { neurons and } \\
\text { motor cortex }\end{array}$ \\
\hline
\end{tabular}

Notes: Modified with permission. Ross CA, Poirier MA. (2004). Protein aggregation and neurodegenerative disease. Nat Med. 10, S10-17.

Disease proteins and associated genes in four major neurodegenerative diseases, together with characteristic pathology and affected brain regions. (modified from Ross and Poirier et al., 2004). 
methyl-D-aspartate (NMDA) receptor antagonists (memantine), or a combination of these agents (donepezil and memantine). Current drugs are dedicated to ameliorate AD symptoms rather than treat the disease itself or delay its progression. Therefore, a breakthrough in research \& development $(\mathrm{R} \& \mathrm{D})$ of AD drugs is urgently needed.

\subsubsection{Alzheimer's Disease: Synapse Disease}

It has been almost a century since Dr. Santiago Ramón y Cajal introduced the notion that dementia may be a result of synaptic degeneration (Cajal SR, 1928). In all AD cases, impairment and loss of synapses is an invariant feature that occurs at early stages. Quantification through electron microscope and immunohistochemical staining using synaptic markers have revealed a significant reduction of synapse density and synaptic proteins in the AD brain, of which hippocampus and cerebral cortex were the two most affected regions (Davies et al., 1987; Bertoni-Freddari et al., 1989; Masliah et al., 2001). Consistently, it had been shown by biochemical and immunohistochemical analysis that both pre-synaptic and post-synaptic components were greatly reduced in AD brains (Reddy et al., 2005). The synaptic loss occurs before neuronal cell loss, suggesting synaptic degeneration is an early event in disease pathogenesis (Davies et al., 1987; DeKosky et al., 1990). Moreover, the degree of impairment in synapse structure and loss of synaptic function are best correlated with the severity of disease in memory decline (Sze et al., 1997; DeKosky et al., 1990; Terry et al., 1991; Shanker and Walsh, 2009).

While $\mathrm{A} \beta$ generation is the beginning of a cascade that leads to $\mathrm{AD}$ pathogenesis, abundant animal studies suggest a strong positive correlation between the levels of $A \beta$ oligomers and the severity of synaptic density loss and cognitive impairment (Haass et al., 2007; Laferla et al., 2007; Shankar and Walsh, 2009). Treatment with secreted A $\beta$ oligomers greatly reduced spine density (Wei et al., 2010). Dendritic spine formation was restored by removing soluble $A \beta$ oligomers with immunotherapy of anti-A $\beta$ antibody in PDAPP transgenic mice supporting the toxicity feature of $A \beta$ on synapse (Spires-Jones et al., 2009). Pathologically increased $A \beta$ oligomers have also been proven to impair synaptic transmission and plasticity. The excitatory synaptic transmission is strictly regulated by two active ionotropic glutamate receptors, NMDA receptors (NMDAR) and AMPA receptors (AMPAR), anchored at the synapse. Increased A $\beta$ oligomers can remove receptor sites responsible for excitatory synaptic transmission, block NMDAR/AMPAR and the reuptake of neurotransmitter glutamate, and shift the activation of the NMDAR-dependent signaling pathway involving long-term potentiation (LTP) induction to the signaling pathway involving long-term depression (LTD) and synaptic loss, although the underlying mechanism remains unclear (Shanker et al., 2007; Li et al., 2009). Increased A $\beta$ oligomers can also destabilize neuronal network activity as evidenced by electroencephalogram (EEG) recordings from cortical and hippocampal networks (Palop and Mucke, 2010). The other AD hallmark is the presence of neurofibrillary tangles containing hyperphosphorylated tau protein. In the studies without tangles, mice with overexpression of human tau displayed synaptic degeneration, showing that the soluble and oligomeric tau is the synaptotoxic species. In both mouse models and human AD brains with tauopathy, neurons with neurofibrillary tangles were 
found not only to have fewer synapses onto their soma, but also to have significantly fewer synaptic proteins than those neurons lacking tangles (Katsuse et al., 2006).

Since synaptic loss and dysfunction represents one of the key features of AD, efforts to protect synapses may be a feasible strategy in treating AD. Studies using insulin-sensitizing compounds (chiro-inositols) demonstrated potential to treat AD by protecting synapses against $\mathrm{A} \beta$ oligomers through insulin mimetic activity (De Felice et al., 2009; Pitt et al., 2013). The dietary ingredient docosahexaenoic acid (DHA) is known to be able to preserve synaptic function and memory in aged individuals. It is also able to function upon $\mathrm{A} \beta$ exposure. Based on the observation of synaptic protection of DHA, Oster and Pillot demonstrated that DHA improved neuronal and brain function in an AD mouse model (Oster and Pillot, 2010). These studies raised the possibility of finding therapeutic strategy focusing on protection of synaptic function in treating neurodegeneration, particularly in AD.

Although $A \beta$ plaques, mainly consisting of fibrillary $A \beta$ aggregates, are the most characteristic feature of $\mathrm{AD}$ pathology, soluble $\mathrm{A} \beta$ oligomers have been discovered to be the main toxic species which adversely affect the synaptic structure and plasticity (e.g. LTP) underlying the pathological mechanism for AD. Additional evidence from clinical trials showed that removing plaques did not reverse neuronal damage, which indicated soluble oligomers, rather than insoluble fibrils, as the primary toxic species. This was also supported by numerous other studies (Klyubin et al., 2005; Kayed et al., 2005; Shankar et al., 2007; Selkoe, 2008). Therefore, future study on treating AD by focusing on protection against synaptic toxicity of soluble $\mathrm{A} \beta$ is promising.

\subsubsection{Mouse Models of Alzheimer's Disease}

In an attempt to better understand the pathological progression of $\mathrm{AD}$, scientists have developed a number of transgenic mouse models based on the known genetic mutations known to be associated with pathological changes, especially in early-onset FAD. The first-ever transgenic mouse model with AD pathology was created two decades ago and many more models were created after that, greatly facilitating basic research on $\mathrm{AD}$ development and drug screening (Cavanaugh et al., 2014). Three different categories of transgenic mouse lines were established based on the two pathological features of $\mathrm{AD}$ : transgenic mouse lines with $\mathrm{A} \beta$ pathology (amyloidopathy), transgenic mouse lines with tau pathology (tauopathy) and transgenic mouse lines with both amyloidopathy and tauopathy.

Distinct mutations within the APP gene: Swedish (K670N/M671L), London (V717I), Indiana (V717F) and Florida (I716V), have been associated with development of amyloid pathogenesis in FAD, and were subsequently named after the places in which they were discovered (Chartier-Harlin et al., 1991; Goate et al., 1991; Eckman et al., 1997). Since then, researchers have developed a number of transgenic mice that express human APP proteins with one or more of these mutations. In general, these transgenic

mice have extracellular $A \beta$ deposits that are developed at different time points throughout 
the mouse lifetime, some followed by neuroinflammation. Furthermore, these transgenic mice present cognitive/memory deficits and behavioral abnormalities. The first generation of transgenic mouse models is monogenic with one or multiple mutations. The first transgenic AD mouse model, named PDAPP, was created by Games et al. in 1995 and had mice expressing APP with Indiana mutation (V717F) driven by platelet-derived growth factor $\beta$ (PDGF- $\beta$ ) promoter. These mice displayed 5-14 fold greater expression of human $A \beta$ than endogenous mouse $A \beta$, and exhibited synapse and memory loss (Games et al., 1995). Dr. Karen Hsiao Ashe developed Tg2576 mouse one year after this APP-expressing model (isoform 695) with the Swedish mutation (K670N/M671L) under the direction of hamster prion protein (PrP) promoter (Hsiao et al., 1996). To date, this is one of the most well-characterized and widely-used AD mouse models. Tg2576 mice display normal cognition early in life, and then develop cognitive deficits during aging (12-24 months). These mice start to show signs of progressive impairment on spatial and learning memory, and also demonstrate deficits in working memory at 12 months, which is considered a presymptomatic AD stage. Memory deficits become worse from 12 to 18 months, which is considered an intermediate symptomatic stage. $\mathrm{Tg} 2576$ mice develop A $\beta$ plaques from 12 months along with some vascular amyloid and dendritic spine loss in hippocampal CA1 region, but no neuronal loss or neurofibrillary tangles containing tau. Synaptic plasticity is also impaired as evidenced by reduced LTP in both the CA1 and dentate gyrus (DG) sub-regions of hippocampus. Eventually, more and more transgenic AD mouse models of first generation were created to overexpress APP with all three (Swedish, London and Indiana) mutations including C3-3 (APPSwe), APP23, J9, TgCRND8 and J20 (Borchelt et al., 1996; Sturchler-Pierrat et al., 1997; Hsia et al., 1999; Mucke et al., 2000; Chishti et al., 2001). Some of the models, such as J20, have more robust $\mathrm{A} \beta$ progression in which learning and memory deficits appear as early as 4 months of age. Moreover, impairment in synaptic plasticity can also be observed in J20 mice between 3 to 6 months of age. Mutations in PSEN are associated with early onset AD in humans. While amyloidopathy in transgenic mice is addressed by creating APP mutations to produce $A \beta$ plaques and cognitive impairment, tau pathology is not presented in these mouse models. To address the pathological role of hyperphosphorylated tau protein and neurofibrillary tangles in AD pathogenesis, researchers have developed AD mouse models that overexpresses human tau protein (MAPT) with certain mutations, such as Tau V337M, PS19 (Tau P301S), Tau P301L, Tg30 (Tau G272V, P301S) (Tanemura et al., 2002; Allen et al., 2002; Terwel et al., 2005; Schindowski et al., 2006). Among them, the PS19 mice is one of the most widely used tauopathy models, carrying tau protein with P301S mutation and developing neuronal loss and cerebral atrophy from 8 to 12 months of age, primarily in the hippocampus and then later spreading to other brain regions. Tau aggregates can be detected as early as 1.5 months of age prior to neurofibrillary tangles formation without any amyloid plaques. PS19 mice also display age-associated cognitive deficits in spatial learning and memory at 7 to 10 months of age. Notably, mutations in the tau protein are increasingly linked to FTD. In an attempt to establish a more ADrelevant tauopathy model, researchers crossed tau knockout mice with human tau protein overexpressing mice, generating a mouse line (Htau) expressing only human tau protein. However, a minimal neurofibrillary tangle was developed in this model (Andorfer et al., 2003). 
By expressing APP mutations and PSEN mutations (M233T, L235P, M146L, L286V), researchers established the second generation of mouse models such as APPSLPS1M146L, APPSLPS1ki, 5×FAD (Tg6799) etc. (Langui et al., 2004; Casas et al., 2004; Oakley et al., 2006). Notably, biogenic or polygenic protein mutations caused early and aggressive amyloid pathology. For instance, $5 \times$ FAD mouse displayed many AD-related phenotypes and demonstrated relatively early $\mathrm{A} \beta$ accumulation and deposits, beginning at around 2 months of age. Plaques are abundantly observable throughout the brain (cortex and hippocampus) by 6 months of age. Synaptic and neuronal loss were also observed at 4 months of age accompanying declining spatial learning ability.

Additionally, to study hereditary cerebral amyloid angiopathy (CAA), researchers created mouse lines, such as APPDutch and ARC48, by overexpressing APP with E693Q (Dutch) or E693G mutations (Herzig et al., 2004; Cheng et al., 2004). In order to replicate both neuropathological features of $\mathrm{AD}$, researchers developed the fourth generation of mouse models, represented by a triple transgenic mouse $(3 \times \mathrm{Tg}-\mathrm{AD})$ expressing human APP protein with Swedish mutations, PSEN with M146V mutation and human tau protein with P301L mutation (Oddo et al., 2003). This mouse model displays both amyloidopathy and tauopathy, with extracellular $\mathrm{A} \beta$ deposits seen at an early time (6 months of age), and tau hyperphosphorylation and aggregates seen at a later time (12-15 months of age). Behavioral changes occur early with cognitive impairment occurring at 4 months of age, and learning and memory deficits at 6.5 months of age. This supports the notion that soluble $A \beta$ oligomers are noxious as functional/behavioral deficits appear prior to the formation of amyloid plaque or neurofibrillary tangles (Morrissette et al., 2009; Schaeffer et al., 2011).

Another approach to enable inducible expression of transgenic protein was established by researchers to create the third generation of AD mouse models, with a representative model named rTg4510 (Santacruz et al., 2005). The rTg4510 mouse model is a tauopathy model that expresses a repressible form of human tau transgene containing the P301L mutation. This transgene is located downstream of a tetracycline (Tet) response element (TRE), and its expression is driven by a second transgene containing tetracycline-controlled transactivators (tTA) under control of a promoter such as CaMKII- $\alpha$ and neurospin (Nop). Tau protein is constitutively induced by tTA and expressed in rTg4510 $\times$ CamkII $\alpha$-tTA mice, and the expression can be turned off by administration of doxycycline (Dox), a tetracycline analogue. This is called Tet-off system. This inducible genetic model enables researchers to investigate half-life and reversibility of phenotypes in FAD. This model displays cognitive impairment by 3 month of age, neurofibrillary tangles by 4 months of age, and synaptic loss by 6 months of age (Santacruz et al., 2005; Spires-Jones et al., 2006).

The fourth generation of AD mouse models features a knock-in approach, with a representative model named APPNL-G-F (Saito et al., 2014). This recently developed approach has the advantage of providing more specific expression patterns and levels, in contrast to potential artifacts produced by general APP overexpression. Moreover it also avoids the potential complication of disrupting unknown genomic loci (Onos et al., 2016). APPNL-G-F mice express APP at wild-type levels with cell-type and temporal specificity. However levels of pathogenic $A \beta$ are elevated due to the combined effects of 
three APP mutations with respective functions: KM670N/M671L (Swedish mutation; promote $A \beta$ toxicity); I716F (Beyreuther/Iberian mutation; increase the $A \beta 42 / A \beta 40$ ratio) and E693G (Arctic mutation; promote $A \beta$ aggregation). This model displays amyloid plaques and gliosis (astrocytosis and microgliosis) at 3 months of age, synaptic loss at 4 months of age, and age-related cognitive impairment (Y-Maze) at 6 month of age (Saito et al., 2014).

AD mouse models for LOAD are more challenging due to the aforementioned environmental factors, and the complex genetic factors, many of which remain unknown. Since APOE is most often associated with LOAD, researchers generated the GFAPAPOE4/APOE KO mouse model which expresses human apolipoprotein E4 (hAPOE4) (Sun et al., 1998). The hAPOE4 is expressed under the direction of the GFAP promoter in the absence of endogenous mouse APOE (mAPOE) by crossing to APOE KO mice. These mice display deficits in $A \beta$ clearance, however neither heterozygous nor homozygous GFAP-APOE4/APOE KO mouse develop complete AD phenotype suggesting the requirement of additional genetic and/or environmental factors (Onos et al., 2016).

AD mouse models are extremely useful tools during various phases of the drug discovery. Rational drug discovery processes begins with target identification and target validation, followed by high-throughput library screening and lead compound optimization. After that, lead compounds are tested in preclinical studies and eventually clinical trials. Among them, AD mouse models are heavily used in the target identification and validation process, as well as in preclinical studies (Hall and Roberson, 2012). Examples of commonly used mouse models of AD are shown in Table 1-2.

\subsection{Neurodegenerative Diseases}

\subsubsection{Heat Shock Response and Heat Shock Proteins}

The heat shock response is a high evolutionarily-conserved response in living cells that protects against intracellular and extracellular stress in all organisms (bacteria, plants, animals). It was firstly discovered in the early 1960s in a Drosophila model by Ritossa and was originally described as the biochemical response of cells to environmental heat (Ritossa, 1962). Later on, researchers discovered this activated heat shock response was also elicited by various other stressors, including environmentally stressed conditions (oxidative stress, low temperature, altered $\mathrm{pH}$, exposure to alcohol, toxins, heavy metals and detergents) and pathological stressed conditions (microbial infections, tissue trauma, genetic lesions) (Schlesinger, 1990; Welch et al., 1991). The heat shock response acts as an essential self-defense mechanism for protecting cells from both intracellular and extracellular harm. A common consequence of cells undergoing various stresses is the misfolding or denaturing of proteins that usually leads to protein accumulation and aggregation. General inhibition of heat shock response results in these aforementioned anomalies. 
Table 1-2 Generations of Commonly Used Mouse Models of AD.

\begin{tabular}{|c|c|c|c|c|c|c|c|}
\hline Generation & Allele(s) & Protein & $\begin{array}{l}\text { Model } \\
\text { name }\end{array}$ & Transgene(s) & Mutation(s) & Promoter(s) & Reference(s) \\
\hline \multirow{15}{*}{$\begin{array}{l}1^{\text {st }} \\
\text { Generation }\end{array}$} & \multirow{15}{*}{$\begin{array}{l}\text { Transgenic } \\
\text { (monogenic) }\end{array}$} & \multirow[t]{9}{*}{$\mathbf{A \beta}$} & PDAPP & hAPP & V717F & PDGF- $\beta$ & Games et al., 1995 \\
\hline & & & $\operatorname{Tg} 2576$ & hAPP & K670N, M671L & Hamster prion protein & Hsiao et al., 1996 \\
\hline & & & $\begin{array}{l}\mathrm{C} 3-3 \\
\left(\mathrm{APP}_{\text {Swe }}\right)\end{array}$ & hAPP & K670N, M671L & $\begin{array}{l}\text { mouse prion protein } \\
\text { promoter }\end{array}$ & $\begin{array}{l}\text { Borchelt et al., 1996; } \\
\text { Savonenko et al., } 2003\end{array}$ \\
\hline & & & APP23 & hAPP & K670N, M671L & murine Thy 1 promoter & $\begin{array}{l}\text { Sturchler-Pierrat et al., } \\
1997\end{array}$ \\
\hline & & & J9 & hAPP & $\begin{array}{l}\text { K670N, M671L, } \\
\text { V717F }\end{array}$ & PDGF- $\beta$ & Hsia et al., 1999 \\
\hline & & & $\mathrm{J} 20$ & hAPP & $\begin{array}{l}\text { K670N, M671L, } \\
\text { V717F }\end{array}$ & PDGF- $\beta$ & Mucke et al., 2000 \\
\hline & & & TgCRND8 & mAPP & $\begin{array}{l}\text { K670N, M671L, } \\
\text { V717F }\end{array}$ & Hamster prion promoter & Chishti et al., 2001 \\
\hline & & & $\mathrm{APP}_{\text {Dutch }}$ & hAPP & E693Q & murine Thy1 promoter & Herzig et al., 2004 \\
\hline & & & ARC48 & hAPP & $\begin{array}{l}\text { K670N, M671L, } \\
\text { V717F ; E693G }\end{array}$ & PDGF- $\beta$ & Cheng et al., 2004 \\
\hline & & \multirow[t]{6}{*}{ Tau } & JNPL3 & MAPT (htau) & P301L & $\begin{array}{l}\text { mouse prion protein } \\
\text { promoter }\end{array}$ & Lewis et al., 2000 \\
\hline & & & $\operatorname{Tg} 214$ & MAPT (htau) & V337M & PDGF- $\beta$ & Tanemura et al., 2002 \\
\hline & & & PS19 & MAPT (htau) & P301S & murine Thyl promoter & Allen et al., 2002 \\
\hline & & & Htau & MAPT (htau) & mTauKO & Tau, Thy1 & Andorfer et al., 2003 \\
\hline & & & Tau P301L & MAPT (htau) & P301L & murine Thyl promoter & Terwel et al., 2005 \\
\hline & & & $\operatorname{Tg} 30$ & MAPT (htau) & G272V, P301S & murine Thy1 promoter & $\begin{array}{l}\text { Schindowski et al., } \\
2006\end{array}$ \\
\hline
\end{tabular}




\section{Table 1-2 (Continued).}

\begin{tabular}{|c|c|c|c|c|c|c|c|}
\hline Generation & Allele(s) & Protein & $\begin{array}{l}\text { Model } \\
\text { name }\end{array}$ & Transgene(s) & Mutation(s) & Promoter(s) & Reference(s) \\
\hline \multirow[t]{5}{*}{$\begin{array}{l}2^{\text {nd }} \\
\text { generation }\end{array}$} & \multirow[t]{5}{*}{$\begin{array}{l}\text { Transgenic } \\
\text { (Bigenic/ } \\
\text { polygenic) }\end{array}$} & \multirow[t]{4}{*}{$\mathbf{A} \boldsymbol{\beta}$} & PS/APP & hAPP, hPSEN1 & $\begin{array}{l}\text { APP K670N, M671L; } \\
\text { PSEN M146L }\end{array}$ & $\begin{array}{l}\text { Hamster prion protein } \\
(\mathrm{APP}), \mathrm{HMGCoA} \\
\text { reductace (PSEN) }\end{array}$ & Holcomb et al., 1998 \\
\hline & & & $\begin{array}{l}\mathrm{APP}_{\mathrm{SL}} \mathrm{PS} 1 \\
\mathrm{M} 146 \mathrm{~L}\end{array}$ & hAPP, hPSEN1 & $\begin{array}{l}\text { APP K670N,M671L, } \\
\text { V717I; PSEN M146L }\end{array}$ & $\begin{array}{l}\text { Thy-1 }(\mathrm{APP}), \mathrm{HMGCoA} \\
\text { reductace }(\mathrm{PSEN})\end{array}$ & Langui et al., 2004 \\
\hline & & & $\mathrm{APP}_{\mathrm{SL}} \mathrm{PS} 1_{\mathrm{ki}}$ & hAPP, hPSEN1 & $\begin{array}{l}\text { APP K670N, M671L, } \\
\text { V717I; PSEN M233T, } \\
\text { L235P }\end{array}$ & Thy1 (APP) & Casas et al., 2004 \\
\hline & & & $\begin{array}{l}5 \times \text { FAD } \\
(\text { Tg6799) }\end{array}$ & hAPP, hPSEN1 & $\begin{array}{l}\text { APP K670N, M671L, } \\
\text { I716V, V717I; PSEN1 } \\
\text { M146L, L286V }\end{array}$ & Thy1 & Oakley et al., 2006 \\
\hline & & $\mathbf{A} \beta /$ tau & $3 \times \mathrm{Tg}-\mathrm{AD}$ & $\begin{array}{l}\text { hAPP, hPSEN1, } \\
\text { MAPT (htau) }\end{array}$ & $\begin{array}{l}\text { APP K670N, M671L; } \\
\text { PSEN1 M146V; tau } \\
\text { P301L }\end{array}$ & $\begin{array}{l}\text { Thyl promoter (APP, } \\
\text { Tau) }\end{array}$ & Oddo et al., 2003 \\
\hline $\begin{array}{l}3^{\text {rd }} \\
\text { generation }\end{array}$ & $\begin{array}{l}\text { Inducible } \\
\text { transgenic }\end{array}$ & Tau & $\begin{array}{l}\text { rTg } 4510 \times \\
\text { CamkII } \alpha- \\
\text { tTA }\end{array}$ & $\begin{array}{l}\text { MAPT } \\
\text { (htau) }\end{array}$ & P301L & CamkII $\alpha$ & Santacruz et al., 2005 \\
\hline $\begin{array}{l}4^{\text {th }} \\
\text { generation }\end{array}$ & Knock-in & $\mathbf{A} \boldsymbol{\beta}$ & $\mathrm{APP} \mathrm{P}^{\mathrm{NL}-\mathrm{G}-\mathrm{F}}$ & hAPP & $\begin{array}{l}\text { APP KM670N, M671L, } \\
\text { APP I716F, APP } \\
\text { E693G (Arctic) }\end{array}$ & APP & Saito et al., 2014 \\
\hline
\end{tabular}

Notes: Modified with permission. Cavanaugh SE, Pippin JJ, Barnard ND. (2014). Animal models of Alzheimer disease: historical pitfalls and a path forward. ALTEX. 31, 279-302. Onos KD, Sukoff Rizzo SJ, Howell GR, Sasner M. (2016). Toward more predictive genetic mouse models of Alzheimer's disease. Brain Res Bull. 122, 1-11. 
Traditional heat shock response is mediated by activation of heat shock transcriptional factors (HSFs). In invertebrates, such as yeast and Drosophila, there is a single heat shock factor (HSF1) mediating transcriptional activation of heat shock response. However, vertebrates have a known family of five HSF members: HSF1, HSF2, HSF3, HSF4 and HSF5. HSF1 can be activated by several stressors and upregulate several heat shock proteins such as Hsp70, Hsp40, Hsp27 etc. In contrast, HSF2 is only activated during early development and differentiation. HSF3 is only expressed in birds. HSF4 functions as a repressor of heat shock proteins. HSF5 is a newly discovered protein and its function remains largely unknown (Fan, 2012). Among these five factors, HSF1 plays the most important role against various stresses. It is essentially a master transcriptional factor in mammalian cells, acting on conserved DNA binding sites called heat shock elements (HSEs). In non-stressed conditions, HSF1 is localized to the cytoplasm in the form of an inactive monomer bound to Hsp90. Under certain stresses, stress-induced activation of heat shock response is completed by disassociation of HSF1 with Hsp90, HSF1 trimerization, phosphorylation, and translocation to the nucleus, where the DNA binding ability of HSF1 is much improved. The nuclear HSF1 interacts with HSEs in the promoter region of target genes that induce protein expression of several heat shock proteins (Sarge et al., 1993) (Figure 1-2).

Heat shock proteins (HSPs) are a group of a multi-gene family of proteins that are highly conserved and ubiquitously expressed in cells. They are also involved in protein transportation, turn-over and regulation. The principle heat shock proteins with chaperon activity are classified into six main sub-families based on their molecular weight: Hsp100, Hsp90, Hsp70, Hsp60, Hsp40 and the small molecular weight heat shock proteins (less than $40 \mathrm{kDa}$ ) (Muchowski and Wacker, 2005). Heat shock proteins function as molecular chaperones to facilitate protein synthesis and assembly, and folding of nascent polypeptides, or correcting misfolded proteins to prevent protein aggregation. Heat shock proteins are also involved in other cellular events, such as protein transportation and degradation, signal transduction, stimulation of innate and adaptive immune systems, cell growth and differentiation, and protection from apoptosis (Nollen and Morimoto, 2002; Lanneau et al., 2008). Different isoforms of heat shock proteins from the same family can localize in different subcellular locations and their expression can be either constitutive or stress-inducible (Lanneau et al., 2008).

\subsubsection{Hsp90 and Hsp70: Structure-based Distinct Functions}

Of all heat shock proteins, Hsp90 and Hsp70 are the two most abundant proteins. Of these two proteins, Hsp90 is more prevalent than Hsp70. Hsp90 has two isoforms, the inducible isoform Hsp90 $\alpha$ (major form) and the constitutive isoform Hsp90 $\beta$ (minor form), which are both constitutively expressed and comprise of $1 \sim 2 \%$ of cellular proteins under non-stressed conditions. Hsp90 exists mainly as a constitutive homodimer ( $\alpha \alpha$ or $\beta \beta)$, but there is also monomer ( $\alpha$ or $\beta$ ), heterodimer $(\alpha \beta)$ and higher oligomers of both isoforms (Sreedhar et al., 2004). Each homodimer is made up of monomers that consist of three main domains: $\mathrm{N}$-terminus domain, middle domain and $\mathrm{C}$-terminus domain. There is also a flexible linker between N-terminus domain and middle domain (M- 


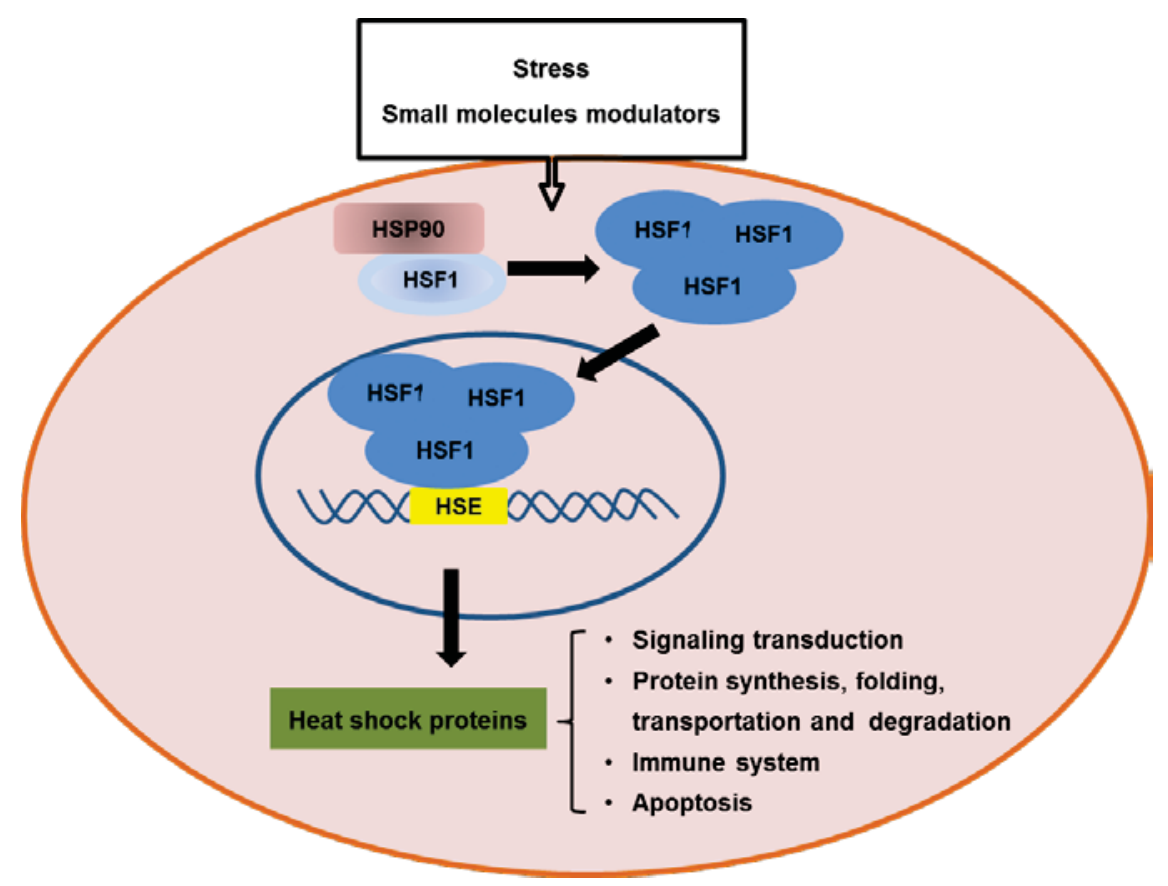

Figure 1-2 HSF1-mediated Heat Shock Response.

Modified with permission. Westerheide SD, Morimoto RI. (2005). Heat shock response modulators as therapeutic tools for diseases of protein conformation. J Biol Chem. 280, 33097-33100.

Heat shock response can be stimulated under both physiological states and pathological states. Stress and small molecules can also cause heat shock response, which is mainly mediated by the master transcriptional regulator HSF1, leading to expression of heat shock proteins. Known functions of heat shock proteins include, but not limit to, regulation of signaling pathway, aiding in protein synthesis, folding, transportation and degradation, activation of immune system, prevention of apoptosis. 
domain). The N-terminus domain contains an ATPase and sites for co-chaperone binding with biochemical functions responsible for the ATPase activity of Hsp90. Importantly it is also the binding site for small molecules including nucleotides, Geldanamycin (GA), 17-AAG, radicicol. The C-terminus domain serves as the docking site for dimerization and co-chaperone binding, such as cdc37, p23 etc. The M-domain is thought to be the major site for client protein binding (Figure 1-3) (Whitesell and Lindquist, 2005). So far, there are more than $200 \mathrm{Hsp} 90$ client proteins identified (Picard, 2008). These client proteins include a large variety of signal transduction molecules, kinases, and receptors involved in controlling cell survival and proliferation. Some notable client proteins are chaperones and relatives (p23, Hsp70, Hsp60, etc.), transcription factors (HSF1, p53, p73, BCL-6, etc.), kinases (AKT/PKB, TrkB, AMPK, Cdk, CamK, MAPK, GSK, MEKK, etc.), and others ( $\beta$-catenin, Cyclin B, Cdc13, HDAC, Huntingtin, tau protein, $\alpha$ synuclein etc.) (Picard, 2008). Many of these proteins are closely related to cancer cell survival and proliferation (Kamal et al., 2004). HSF1 is a client protein of Hsp90 (Zou et al., 1998). Upon inhibition of Hsp90, HSF1 is released for transcriptional activation (Taipale et al, 2010) and induces protein expression of inducible Hsp70. Given the wellknown function of Hsp90 on stabilizing client proteins, Hsp90 inhibition was also found to promote protein degradation through the ubiquitin-proteasome (UPS) pathway (Li and Buchner, 2013).

The structure of Hsp70 contains an N-terminus domain, which is an ATPase domain/Nucleotide binding domain (NBD), and a C-terminus domain that is also the substrate binding domain (SBD) (Evans et al., 2011). There are two isoforms of Hsp70, heat shock cognate protein 70 (HSC70) and inducible form of Hsp70 (iHsp70). HSC70 is constitutively expressed, while inducible Hsp70 (iHsp70) is poorly expressed in resting conditions or induced to express in stressed conditions. As a major molecular chaperone,

The CBD of Hsp70 is a key component in folding catalysts that aids in a wide variety of protein processes including folding of freshly synthesized protein, protein assembly, refolding of misfolded proteins and protein aggregates, protein translocation in membrane and control of regulatory proteins' activity. Thus, Hsp70 has a housekeeping function in mammalian cells that governs general protein quality control. This activity appears to rely on the transient binding interaction of the substrate binding domain (SBD) with hydrophobic short peptide segments of the substrate proteins controlled by Nterminus ATPase domain (Mayer and Bukau, 2005). The roles of Hsp70 in folding newly synthesized proteins can be separated into the following 3 activities: 1) prevention of protein aggregation which is completed with its co-chaperone of the J-domain protein; 2) facilitating folded proteins to the native state (mechanism remains unclear); 3) refolding or solubilization of aggregated proteins, which is performed through mutual cooperation with chaperone proteins of the Hsp100 family (Hsp104/ClpB) (Mayer and Bukau, 2005).

While these two major chaperone proteins, Hsp70 and Hsp90, are both richly localized in synaptic compartments (Moon et al., 2001; Stetler et al., 2010), little is known regarding their synaptic functions. Given that there is a common misconception of targeting Hsp90 inhibition on treating human diseases, such as cancers and neurodegenerative diseases, Hsp70 inhibition is indeed thought to be able to constrain 


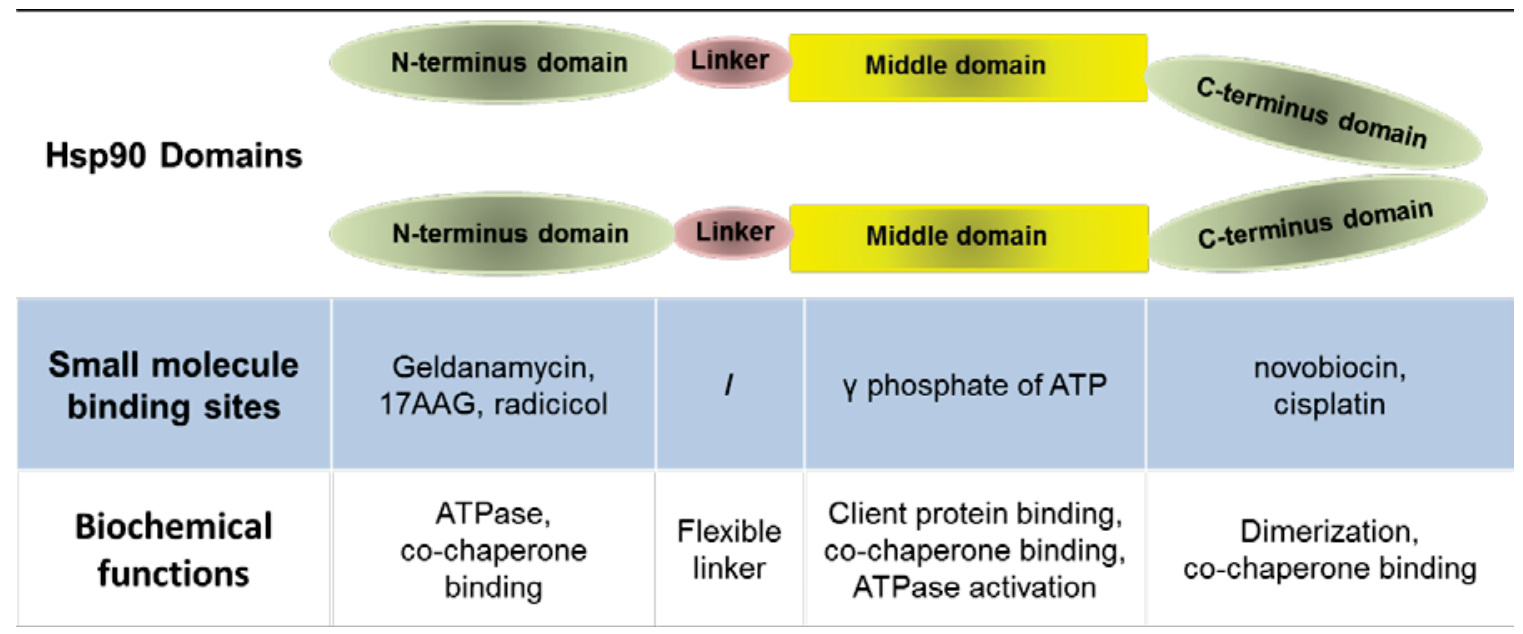

Figure 1-3 Structure and Functions of Hsp90 Dimer.

Modified with permission. Whitesell L, Lindquist SL. (2005). Hsp90 and the chaperoning of cancer. Nat Rev Cancer 5, 761-772.

The protein structure, location of binding sites and their biochemical functions are indicated for HSP90. 
heat shock response. In contrast, Hsp90 inhibition not only promotes degradation of client proteins, but also causes HSF1-mediated heat shock-like response activation along with other important synaptic regulation machinery, as discovered by the current study.

\subsubsection{Heat Shock Proteins in Neurodegeneration}

To maintain cellular integrity of the proteome, the normal protein quality control process determines whether an existing protein should be folded or degraded, and ensures that the newly synthesized protein undergoes the correct folding rather than misfolding and/or aggregation. Neurodegenerative diseases (AD, PD, HD, FTDP, etc.) are commonly characterized by abnormalities in the process of protein quality control, leading to aberrant formation of distinct pathological misfolded proteins and/or protein aggregates. In addition to those listed in Table 1-1, other disease-causing deposited proteins include hyperphosphorylated tau in FTD with Parkinsonism; Atrophin-1, ataxins or androgen receptor (AR) in other polyglutamine diseases (Ross et al., 2004). Since most pathological events start with formulation of these protein aggregates, targeting clearance of these misfolded proteins and/or protein aggregates is a logical therapeutic idea for treating neurodegenerative diseases.

While the clearance of misfolded proteins and/or protein aggregates following Hsp90 inhibition is ill-defined, it is generally accepted that it requires induction of a heat shock-like response involving HSF1-mediated upregulation of other chaperone proteins such as iHsp70 and its co-chaperones (e.g., Hsp40/DNAJ, Hsp27, etc.). Studies have shown the co-localization of various chaperone proteins (such as Hsp70) with protein aggregates that are characteristic of $\mathrm{AD}, \mathrm{PD}, \mathrm{FTDP}$ and the polyglutamine disease (Muchowski et al., 2005). Protein overexpression of select heat shock proteins (Hsp70, Hsp40 and Hsp27) has exhibited a certain level of neuroprotection in several animal models of neurodegenerative diseases. For instance, Hsp70 overexpression has been shown to protect neurons from $\mathrm{A} \beta$ toxicity in an in vitro $\mathrm{AD}$ model (Magrané et al., 2004). Hsp70 overexpression can also protect neurons from $\alpha$-synuclein mediated toxicity in a drosophila model of PD (Auluck et al., 2002). The aforementioned neuroprotective observations were due to elevation of a single heat shock protein. Induction of a set of various heat sock proteins can be more potent in neuroprotection than overexpression of one type of heat shock protein. Heat shock proteins may show neuroprotection via preventing interactions of misfolded protein and promotion of protein degradation (Brown, 2007). In mammalian cells, the master transcription regulator HSF 1 controls induction of stress-induced heat shock proteins. It thus would be promising to discover a strategy to regulate heat shock proteins via HSF1 for neurodegenerative diseases that produce misfolded proteins and/or protein aggregates (Neef et al., 2011).

\subsubsection{Hsp90 Inhibitors}

Since many Hsp90 client proteins control various important cellular events such as cell survival, proliferation and apoptosis, Hsp90 function is closely associated with 
health. In fact, Hsp90 has been found to play an important role in disease progression. For instance, Hsp90 was found to be constitutively overexpressed at intracellular levels 2 to 10-fold greater in tumor cells compared to normal cells (Ferrarini et al., 1992) and the inducing molecule including NF- $\mathrm{B}$ (Ammirante et al., 2008) Hence, Hsp90 appears to be an important target in cancer therapy. Various natural and synthetic Hsp90 inhibitors have been developed for preclinical anti-tumor activity. Common Hsp90 inhibitors target the ATP binding site at the $\mathrm{N}$-terminus inactivating ATPase, ultimately resulting in release of Hsp90 client proteins such as HSF1.

There are two major classes of Hsp90 inhibitors: ansamycin backbone based inhibitors such as geldanamycin (GA) and its derivatives, and radicicol based inhibitors including radicicol and its derivatives. GA was firstly discovered by DeBoer et al. in the early 1970s in the broth and the mycelium of Streptomyces (DeBoer et al., 1970). The chemical structure of GA was established and reported by Sasaki et al. later that year (Sasaki et al., 1970). The antitumor effects of GA were reported 7 years later by two different groups through in vitro studies (Li et al., 1977; Price et al., 1977). For a long time, little to no progress was made, that is until a breakthrough discovery made by Ferrarini et al. in 1992, which elucidated the role of heat shock proteins in cancer progression (Ferrarini et al., 1992). GA was found to be able to restructure cancer-related proteins (Blagosklonny et al., 1995). GA was then introduced to be used in animal studies. However, GA exhibited high toxicity in the liver, kidney and other organs, too extreme for in vivo use (Supko et al., 1995), so researchers focused on generation of GA derivatives with less toxicity. 17-AAG (17-N-allylamino-17-demethoxygeldanamycin, also known as tanespimycin) was synthesized and found to possess lower in vivo toxicity and better stability than GA. Although Hsp90 binding affinity for 17-AAG was weaker than for GA, 17-AAG exhibited biologic antitumor activity at almost the same concentrations with GA in malignant cell lines (Schulte and Neckers, 1998). In 1999, 17 AAG entered the clinic trials for cancers. Due to the poor chemical solubility of 17-AAG, another GA derivate named 17-DMAG (17-Dimethylaminoethylamino-17-

demethoxygeldanamycin, also known as alvespimycin) was developed with better solubility and entered first human study in 2004.

The radicicol backbone based Hsp90 inhibitors do not cause hepatotoxicity. Although, radicicol is not suitable for therapeutic applications due to in vivo instability (Soga et al., 1999), researchers have thus focused on the development of radicicol derivatives with improved stability. NexGenix Pharmaceuticals (now acquired by Oncosynerge. Inc.) engineered and synthesized a pochoxime series of compound with the leading compound NXD30001 (new name OS47701) and OS47720 (also known as pochoxime C) (Barluenga et al., 2009; Wang et al., 2009; Zhu et al., 2010). NXD30001 displayed in vivo stability and an improved safety profile. It is also CNS-permeable and binds with Hsp90 with high affinity. NXD30001 induced expression of heat shock proteins (iHsp70, Hsp60, Hsp40 and Hsp27) in a dose, time and HSF1-dependent manner with a comparable efficacy to17-AAG in breast cancer cells (Cha et al., 2014).

Hsp90 inhibitors were first introduced in treating neurodegenerative diseases by Waza et al. in 2005, who reported 17-AAG's ability to ameliorate motor impairments in 
the spinal and bulbar muscular atrophy (SBMA) transgenic mouse model, through induction of heat shock protein expression and reduction of monomeric and aggregated mutant androgen receptor (AR) (Waza et al., 2005). Two other groups (Luo et al., 2007; Dickey et al., 2007) have also targeted Hsp90 inhibition in tau pathology by using synthesized Hsp90 inhibitors, and both groups were able to observe reduction of aggregated tau. However, no follow-up study demonstrating in vivo efficacy with improved symptoms has been reported, assuming poor or very limited efficacy produced from these in vivo applications. More recent studies of NXD30001 have shown induction of heat shock proteins and protective profiles against mutated SOD1 in motor neuron culture models of fALS (Cha et al., 2014). We therefore proceeded to evaluate the therapeutic effects of the Hsp90 inhibitor, OS47720, in both an in vitro cell culture models and an in vivo Alzheimer's disease mouse model.

\subsection{Hypothesis and Rationale}

\subsubsection{Hypothesis}

We hypothesize that OS47720 may be a feasible Hsp90 inhibitor suitable for further drug development as an AD therapeutic agent whose actions are HSF1-dependent.

\subsubsection{Rationale}

Alzheimer's disease (AD) is a progressive neurological disorder afflicting millions of individuals worldwide. Current therapeutic approaches focus on reducing $A \beta$ levels via inhibiting $\beta$ - and $\gamma$-secretases, but have yielded minimal clinical success (Becker et al., 2008; Honig, 2012). A family of chaperone proteins has been shown to be involved in the protein assembling and disassembling processes. Nowadays, the importance of chaperones in the quality control of proteins has been widely understood. Hsp90, as one of the most abundant of the chaperone protein family, functions in assisting proper protein folding, stabilizing proteins against cellular stress, and aiding protein degradation (Young et al., 2001). Because many aberrant proteins involved in tumor growth and survival (e.g., protein kinase B/AKT) are also stabilized by Hsp90, Hsp90 inhibitors have long been recognized as candidate drugs for cancer treatment (Whitesell and Lindquist, 2005; Neckers, 2007). Hsp90 is also implicated in neurodegenerative diseases (Luo et al., 2010), such as AD) and PD. Several reports also showed that Hsp90 inhibitors reduced abnormal protein aggregations of tau (Dickey et al., 2007) and $\alpha$-synuclein (Putcha et al., 2010), possibly via the activated heat shock proteins (e.g., iHsp70 etc).

Since both Hsp90 and Hsp70, the two most abundant forms of chaperone proteins, are localized at synapses and around amyloid plaques, Dr. Liao's group has sought out to study the effects of Hsp90 inhibitors in synaptic functions, beginning as early as 2008. Dr. Chen, a former graduate student in her lab, made the initial discovery of a novel aspect of 
the Hsp90 inhibitor, using a commercial compound 17-AAG in protecting primary neurons from soluble $A \beta$-induced synaptic toxicity. I participated in that project and was able to later reproduce Dr. Chen's in vitro observations, in which 17-AAG was found to induce heat shock protein, upregulated presynaptic and postsynaptic proteins in cultured neurons, and preserved structure of synapses against $A \beta$ toxicity. I was able to further discover 17-AAG's neuroprotective effect in preventing loss of contextual conditioned freezing memory induced by soluble A $\beta$-impaired in contextual fear conditioning assay. Together, we published a brief report in "The Journal of Neuroscience". Since 17-AAG has limited CNS-permeability (Zhu et al., 2010), I then tested the CNS-permeable compound OS47720, obtained from a private Oncology company, in both in vitro and in vivo studies. In the in vitro study, OS47720 induced a heat shock-like response comparable to that induced by 17-AAG (e.g., upregulated not only multiple HSPs but also pre- and post-synaptic proteins), and furthermore protected loss of synaptic proteins and dendritic spines induced by soluble A $\beta$. Most importantly, OS47720 had no observable hepatotoxicity, as was not the case with GA or 17-AAG. The efficacy of OS47720 in an AD mouse model (Tg2576) was further examined. Given that OS47720 targets Hsp90 inhibition by releasing HSF1, HSF1-mediated transcriptional and synaptic regulation was further examined.

\subsubsection{Specific Aims}

Aim 1. To test whether the Hsp90 inhibitor 17-AAG shows a neuroprotective effects against $\mathrm{A} \beta$ toxicity both in vitro and in vivo.

Aim 2. To test OS47720's pharmacological and in vivo efficacy in early and middle symptomatic Tg2576 mice.

Aim 3. To investigate the molecular mechanism of HSF1 mediated regulation underlying OS47720's therapeutic effects. 


\section{CHAPTER 2. MATERIALS AND METHODS}

\subsection{Animal Studies}

\subsubsection{Mouse}

All animal procedures were performed in accordance with the Animal Scientific Procedures Act and with the approval of the Institutional Animal Care and Use Committee (IACUC) at the University of Tennessee Health Science Center (UTHSC). The breeding pair of Tg2576 mice was originally purchased from Taconic (Stock\#: 1394) and maintained on a C57BL6/J background. The breeding pair of HSF1 mice was ordered from the Jackson laboratory (Stock\#: 018582) and maintained on the C57BL6/J background. The Hsp70-mPlum reporter mouse line was generated from Dr. Anna M. Planas (Institute for Biomedical Research of Barcelona, Spain) and provided to us via collaboration.

\subsubsection{Genotyping}

Tail tissue (about $2 \mathrm{~mm}$ ) from young $(<21 \mathrm{~d}$ ) mice (without anesthesia) or adult $(>$ $21 \mathrm{~d}$ ) mice (with topical anesthesia) was taken by using a sterilized scissors. Tissue was immediately used for DNA extraction or kept frozen at $-20^{\circ} \mathrm{C}$ for future use. DNA was isolated following a phenol-chloroform extraction protocol (The Jackson laboratory, Inc.). PCR was performed with KAPA Mouse Genotyping Kits (Kapa Biosystems, Inc.). $\operatorname{Tg} 2576$ mice were genotyped by PCR with the following primer sequences $\left(5^{\prime}->3^{\prime}\right)$ : Tg2576 transgene (transgenic) forward AGG ACT GAC CAC TCG ACC AG; Tg2576 transgene reverse CGG GGG TCT AGT TCT GCA T; Tg2576 internal positive control (wild type) forward CTA GGC CAC AGA ATT GAA AGA TCT; Tg2576 internal positive control reverse GTA GGT GGA AAT TCT AGC ATC ATC C. HSF1 mice colony were genotyped by PCR with the following primer sequences: HSF1 mutant forward CGG TCG CTA CCA TTA CCA GT; HSF1 mutant reverse CCA AGG AGA TGT AGT GAG GTC TG; HSF1 wild type forward CTC CAT CTC CAG CCT ACA GC; HSF1 wild type reverse AGA GTG CTG CTG GAC AGA GG.

\subsubsection{Intracerebroventricular (i.c.v.) Cannulation}

Surgery was performed under aseptic conditions in a stereotactic instrument (Stoelting Co., Wood Dale, IL, USA). Mice were anesthetized by intraperitoneal injection of xylazine/ketamine $(13 / 87 \mathrm{mg} / \mathrm{kg})$. Ketamine supplements $(10 \mathrm{mg} / 0.1 \mathrm{ml})$ were given intraperitoneally (i.p.) as needed throughout surgery. A small incision was made, and the skull was then exposed and cleaned and a small burr hole was drilled using a dental drill or Dremal rotary tool. One unilateral guide cannula (Plastics One; C315GS-5-SPC, cut $1.5 \mathrm{~mm}$ below the pedestal) was implanted above the dorsal lateral ventricle using a 
stereotaxic instrument (KOPF Instruments, CA, USA) at the coordinates: $\mathrm{AP}=-0.37 \mathrm{~mm}$; $\mathrm{ML}=+1.00 \mathrm{~mm}$; $\mathrm{DV}=-1.5 \mathrm{~mm}$ with respect to bregma. A cannula was mounted to the skull with medical grade superglue (Loctite 454). A paired dummy was inserted into the guide cannula to prevent clogging (Plastics One; C315DCS-5-SPC, cut to completely fill the guide cannula). After the surgery, all mice received one-time intraperitoneal injection of buprenorphine $(0.1 \mathrm{mg} / \mathrm{kg})$ and sterile saline $(0.5 \mathrm{ml})$ for analgesia and rehydration respectively. More injections were given based on symptoms of pain and discomfort during recovery. Mice were allowed to recover from the surgery for at least 5 days before i.c.v. injections and behavioral testing. Implanted cannulas and animals were monitored on a daily basis.

\subsubsection{Intracerebroventricular Injection}

The 7PA2 CM, 17-AAG, OS47720 or vehicle [Chinese Hamster Ovary (CHO) $\mathrm{CM}$ for $7 \mathrm{PA} 2 \mathrm{CM}$ or $100 \%$ DMSO for 17-AAG and OS47720] alone, was administered i.c.v. to anesthetized C57 mice in a total volume of $2 \mu \mathrm{l}$. The 17-AAG compound, or vehicle, was injected using a Hamilton syringe with a 27 -gauge needle (Reno, NV, USA) at a rate of $0.5 \mu \mathrm{l} / \mathrm{min}$ into the planted canula after removal of the dummy cap.

\subsubsection{Intra-hippocampal CA1 Viral Microinjection}

Intra-hippocampal CA1 injection of viruses was similar to cannulation. Instead of implanting cannulas, a KOPF microinjection unit (Model\#: 5000) was used to inject $1 \mu 1$ of $\mathrm{AAV}$ viruses into each of the $\mathrm{CA} 1$ region at the coordinates: $\mathrm{AP}=-2 \mathrm{~mm} ; \mathrm{ML}=+1.5$ $\mathrm{mm}$; DV $=-1.75 \mathrm{~mm}$ with respect to bregma, with a speed of $0.2 \mu \mathrm{l} / \mathrm{min}$ for 5 minutes. Syringes (27-gauge needle) will be remained for an additional $3 \mathrm{~min}$ for drug diffusion. Virus-injected mice were housed for 3 weeks before tests to let the virus reach expression peak. In mice with both i.c.v. cannulation and intra-hippocampal CA1 microinjection, both surgical procedures were performed at the same time. Virus expression peak was determined by examining expression of EGFP at 1,2,3 and 4 weeks by sectioning brain slices $(10-15 \mu \mathrm{m})$ after injection.

\subsubsection{Compound Administration}

The solvent used for OS47720 administration consisted of $6 \%$ dimethylacetamide (DMA), 5\% Tween-80 in sterile H2O. The drug compound was injected through i.p. twice a week for the first 4 weeks, then three times a week until the end of study. Treatment started at 9-months Tg2576 mice and lasted for 3 months, or at 12-months Tg2576 mice and lasted for 6 months. 


\subsubsection{Behavioral Tests}

2.1.7.1 Contextual Fear Conditioning. The Contextual fear conditioning test was conducted as previous described (Chen et al., 2014; Kittelberger et al., 2012). Briefly, mice were placed in a rectangular box with steel walls and a steel grid floor $(30 \mathrm{~L}$ x $30 \mathrm{~W}$ x $25 \mathrm{H}, \mathrm{cm}$ ) and video captured by overhead cameras of the four-chamber fear conditioning system (Coulbourn Instruments LLC). Fear acquisition was performed on Day 1 and contextual fear retention (24, 48 and 72 h) was performed on Day 2-4. During fear acquisition, three minutes baseline recording was given followed by a $20 \mathrm{~s}$ tone $(2.8$ $\mathrm{kHz}, 82 \mathrm{~dB})$ and a $2 \mathrm{~s}$ footshock $(0.7 \mathrm{~mA})$. One minute of recording was taken before the second similar tone-shock stimulus (a total of two presentations of the tone-shock pairing). Mice were recorded for another 1 min till the end. During contextual fear retention test, three minutes of recording was performed. Time intervals analyzed for the fear acquisition are as follows: minute $1(\mathrm{~m} 1)$ : 0-60 s; minute $2(\mathrm{~m} 2)$ : 60-120 s, minute 3 (m3): 120-180 s; tone 1 (t1): 180-200 s; inter-tone interval (iti): 200-260 s; tone 2 (t2): 260-280 s and end: 280-340 s. Time interval analyzed for the context fear retention is context from 60 to $180 \mathrm{~s}$. Freezing percentage was analyzed by FreezeFrame software (Coulbourn Instruments LLC). A higher freezing percentage in contextual retrieval test reflects better memory.

2.1.7.2 Open Field Test. Each animal was individually placed in a novel openfield arena $(47 \mathrm{~L} \times 37 \mathrm{~W} \times 20 \mathrm{H}, \mathrm{cm}$; 900 lux at the center; Accuscan Instruments) for 10 min while being recorded by an overhead camera. Total locomotor activity was measured by beam breaks of the equipped 16 infrared beams. The footage was then analyzed by an automated tracking system (EthoVision XT software). The time spent in the center $(25 \%$ of the field) was measured as a potential indicator for anxiolytic behavior.

2.1.7.3 Open-field Locomotor Activity. Exploratory locomotor activity was measured in an open field measuring $27 \times 27 \mathrm{~cm}$. Infrared beams and detectors automatically record movement in the open field. A 10-min session was given, in which the mouse was placed in the activity monitor and removed 10 min later. No discrete stimuli or motivators were applied, and there were no imposed contingencies to manipulate behavior. Total distance travelled was recorded as locomotor activity.

2.1.7.4 Elevated Plus Maze. The elevated plus maze (EPM) apparatus is a "+"shaped maze with two facing open arms, two facing closed arms and a center area (arm/wall sizes: $50 \mathrm{~L} \times 10 \mathrm{~W} \times 30 \mathrm{H}$, cm; Runway height from floor: $55 \mathrm{~cm}$ ). Each mouse was allowed to freely explore the maze for $5 \mathrm{~min}$. The preference for being in open arms over closed arms (expressed as a percentage of time spent in the open arms) was calculated to measure anxiety-like behavior. 
2.1.7.5 Novel Object Recognition. The task was conducted in an open arena (47 $\mathrm{L} \times 37 \mathrm{~W} \times 20 \mathrm{H}$ ) with 2 objects in HSF1 heterozygous mice. During habituation (day 1), mice were allowed to freely explore the arena. On day 2, mice were exposed to 2 identical objects (red cubes) placed in the center of the field. On day 3, 1 object was placed by another novel object (blue triangle). Each test lasted for 10 minutes. Time spent in exploring each object was recorded. Exploration time and discrimination index (= time spent in novel object / total exploration time x 100\%) were calculated.

2.1.7.6 Cross Maze. Spontaneous alternation rates were assessed using a crossmaze. Cross maze was conducted in a "+"-shaped maze with four white and opaque plastic arms with walls (arm/wall sizes: $50 \mathrm{~L} \times 10 \mathrm{~W} \times 30 \mathrm{H}, \mathrm{cm}$ ). Animals were introduced from a fixed entry located in one of the four arms, and were allowed to freely explore four arms for $5 \mathrm{~min}$. An alternation is defined as going into each of the four arms without repeating an arm. The number of alternations is then divided by the number of arm choices minus three to yield the alternation rate, which is a measure of working memory.

2.1.7.7 Morris Water Maze. Morris water maze (MWM) test was conducted in a 118 -cm diameter round tank, filled with $25^{\circ} \mathrm{C}$ opaque water using non-toxic white tempera paint (Crayola). During the test, mice were first trained to learn swimming and to find cued (visible by a black acrylic ball $30 \mathrm{~cm}$ above the platform) escape platform (10 $\mathrm{cm}$ diameter; $0.5 \mathrm{~cm}$ above water surface) for 3 days, then underwent 10 days of hidden platform session $(0.5 \mathrm{~cm}$ below water surface) and 1 additional day of probe trial session (platform removed). Two days of rest were given before mice went to hidden platform session. During the hidden platform session, swimming path and distance was recorded and calculated as measures of spatial and learning memory. During the probe trial session, hidden platform was removed. Platform crossings and time spent in a $40-\mathrm{cm}$ zone centered on the former platform location were recorded as measures of spatial memory.

\subsubsection{Perfusion and Fixation for Histology}

For each surgery, we set up a perfusion syringe and attached a perfusion set with perfusion needle. Surgical scissors, forceps and clamps were prepared sterile with autoclave. $15 \%$ isoflurane (in mineral oil) was given to the animal. Once the animal was under anesthesia, we placed it on the operating pan with its back down. We used some tape to hold the appendages so that the animal was securely fixed. The pinch-response method was used to determine depth of anesthesia. An incision with scalpel was made through abdomen the length of the diaphragm. With sharp scissors we cut through the connective tissue at the bottom of diaphragm to allow access to rib cage. With large scissors, we cut through ribs just left of the rib cage midline. One center or two end horizontal cuts were made through the rib cage to open up the thoracic cavity. Clamp open to expose heart and provide drainage for blood and fluids. While holding heart 
steady with forceps (it should still be beating), needle was inserted directly into protrusion of left ventricle to extend straight up about $4 \mathrm{~mm}$. Needle position was secured by clamping in place near the point of entry. A steady flow of around $20 \mathrm{ml} / \mathrm{min}$ of PBS was manually injected. A cut in atrium was made with sharp scissors, and solution was flowing freely. If fluid was not flowing freely or was coming from animal's nostrils or mouth, the needle was repositioned. When blood had been cleared from body, solution was changed to 4 percent paraformaldehyde (PFA) solution (30-60 $\mathrm{ml} / \mathrm{mouse})$. Perfusion was almost complete when spontaneous movement (formalin dance) and lightened color of the liver were observed. The perfusion was stopped and the brain and other organs of body were collected. Tissues were placed in vials containing the same fixation and fix for another 2 more hours on ice or at 4 degree before proceeding to dehydration and embedding.

\subsection{Biochemical Studies}

\subsubsection{Primary Cell Culture}

Primary cortical and hippocampal neurons were isolated and purified from E17 embryos of Sprague Dawley (SD) rats. Isolated primary neurons were either plated onto cover slips at a low density of $1 \times 104$ per well in 24-well plates for staining, or plated onto 6 -well plates at a density of $6 \times 105$ per well, and $100 \mathrm{~mm}$ dishes at a density of $3 \times$ 106 per dish for Western Blot analysis. All plates were pre-coated with poly-D-lysine $(100 \mu \mathrm{g} / \mathrm{ml})$. The cultures were maintained in serum-free Neurobasal medium (Invitrogen) with $2 \%$ B27 supplement (Invitrogen).

\subsubsection{Immunocytochemistry and Dendritic Spine Quantification}

Rat hippocampal neurons were cultured for 21 days at low density were fixed by $4 \%$ PFA, permeabilized by $0.1 \%$ Triton X-100, and immuno-stained for synaptic proteins and visualized using a confocal fluorescence microscope. Anti-PSD95 (1:100; Santa Cruz Biotechnology), anti-Synapsin I (1:200; Cell signaling) antibodies and DAPI (Roche) for nuclear staining were used. Synaptic clusters were calculated as the average number of clusters per $10 \mu \mathrm{m}$ of dendritic length. AAV1-YFP was used to infect neurons (DIV 14) for 7 days (DIV 21) to highlight dendritic spines of hippocampal neurons at low density. Dendritic spines of primary and secondary basilar dendrites were counted and analyzed. Images were captured under a confocal microscope (Olympus). $488 \mathrm{~nm}$ and $594 \mathrm{~nm}$ wavelengths were used. Fluorescent intensity was analyzed by ImageJ software (NIH). 60x object magnification was used to acquire images. 


\subsubsection{Protein Analysis}

Cells were lysed in ice-cold lysis RIPA buffer, consisting of $50 \mathrm{mM}$ Tris- $\mathrm{HCl} \mathrm{pH}$ 8.0, $150 \mathrm{mM} \mathrm{NaCl}, 1 \%$ Triton X-100, 0.5\% sodium deoxycholate, 0.1\% SDS, and a mixture of protease and inhibitors (Thermo Scientific, Inc). The protein concentration of each sample was determined by using Micro BCA Protein Assay Kit (Thermo Scientific) and read by Multimode plate reader (Beckman Coulter DTX 880). Samples were prepared and separated on NuPAGETM 4-20\% Tris glycine gel (Life Technology) and transferred to a polyvinylidene difluoride (PVDF) membrane (Millipore). Antibodies (Abs) were used as follows: rabbit anti-Hsp90, cHsp70, Hsp40, Hsp27, HSF1, PSD95, Synapsin I (1:1000; Cell Signaling); mouse anti-iHsp70 (1:1000; Enzo Life Sciences), rabbit anti-BDNF (1:500; Santa Cruz Biotechnology); mouse anti- $\beta$-actin (1:10000; Sigma-Aldrich), rabbit anti-Synaptophysin (1:2000; Chemicon/Millipore), and antimouse IgG and anti-rabbit IgG horseradish peroxidase-conjugated Abs (1:5000; SigmaAldrich). The membranes were washed three times for 15 min each with TBST containing $0.05 \%$ Tween 20 ( $\mathrm{pH} \mathrm{7.4)} \mathrm{and} \mathrm{incubated} \mathrm{with} \mathrm{horseradish} \mathrm{peroxidase-}$ conjugated secondary antibodies in 5\% skim milk for 1 hour at room temperature. After washing three times with TBST, immunoreactive bands were visualized by using ECL Plus (Thermo Scientific) chemiluminescence reagent.

\subsubsection{Naturally Aecreted A $\beta$-containing Conditioned Medium}

Conditioned medium $(\mathrm{CM})$ containing naturally secreted $\mathrm{A} \beta$ oligomers was collected from cultures of 7PA2/CHO cells as previously reported (Walsh et al., 2002; Li et al., 2014). The 7PA2 cells are Chinese Hamster Ovary (CHO) cells which stably express human APP751 which contains a Val717Phe mutation (an APP isoform of 751 amino acids in length). Medium containing soluble human A $\beta$, called 7PA2 CM, was derived from the conditioned medium of 7PA2 cells. Briefly, 7PA2 cells were grown in Dulbecco's modified Eagle's medium (DMEM, HyClone) with 10\% characterized fetal bovine serum (FBS; HyClone, Inc) and $200 \mu \mathrm{g} / \mathrm{ml} \mathrm{G418} \mathrm{(Calbiochem,} \mathrm{Inc)} \mathrm{until} \mathrm{80 \%}$ confluence. Cells were then washed with sterilized PBS and conditioned in $5 \mathrm{ml}$ of B27free neurobasal medium (Invitrogen, Carlsbad, CA) for about $16 \mathrm{hr}$. Afterwards, 7PA2 $\mathrm{CM}$ was removed and cleared of floating cells and debris by passage through a sterile $0.22 \mu \mathrm{M}$ filter (EMD Millipore, Inc). The control medium, called CHO CM, was derived similarly from $\mathrm{CHO}$ cells cultured in DMEM containing 10\% FBS and G418. Aliquots of 7PA2 $\mathrm{CM}$ and $\mathrm{CHO} \mathrm{CM}$ were stored at $-80^{\circ} \mathrm{C}$ Before use; the $\mathrm{CM}$ was supplemented with $2 \% \mathrm{~B} 27$ and $0.5 \mathrm{mM}$ glutamine. $\mathrm{CM}$ was used at original concentration for lateral ventricle microinjection, and was diluted at 1:1 with respective neurobasal culture medium to treat neurons.

\subsubsection{RNA Analysis (PCR and qRT-PCR)}

Total RNA was extracted from cells by using Trizol reagent (Life Technology). The High-Capacity cDNA Reverse Transcription Kit (Applied Biosystems) was used to 
synthesize the first strand cDNA from the samples with an equal amount of RNA, according to the manufacturer's instructions. Synthesized cDNAs were then amplified and analyzed on Real-Time PCR Systems (Invitrogen) using SYBR Green MasterMix (5 Prime, Inc.). Primers used were listed in Table 2-1.

\subsubsection{Histology and Immunohistochemistry}

Mice were heart-perfused by saline and 4\% paraformaldehyde (PFA), post-fixed in $4 \%$ PFA overnight as detailed above. Mouse brains were paraffin-embedded, sectioned at $5 \mu \mathrm{m}$, and mounted on glass slides. The $5 \mu \mathrm{m}$ sections were deparaffinized by passing through 100\% xylene and then passing through serial dilutions of ethanol $(100,95$, and $75 \%$ ). Hematoxylin and Eosin (H\&E) staining was performed and images were captured under a light microscope. For Optimal Cutting Temperature compound (OCT) embedding, perfused brains were cryoprotected by $30 \%$ sucrose infiltration and then sectioned at $10 \mu \mathrm{m}$ using a cryostat (Leica). Anti-HSF1 (1:100; cell signaling), anti- $\beta$ Amyloid (clone 4G8; 1:100; Covance), anti-GFAP (1:1000; Sigma) and anti-Iba1 (1:500; Wako) antibody were used and nuclei were stained with DAPI. Goat anti-Rabbit IgG secondary antibody, Alexa Fluor ${ }^{\circledR} 594$ conjugate and anti-mouse IgG sencondary antibody, Alexa Fluor ${ }^{\circledR} 488$ conjugate (Thermofisher Scientific) were used.

\subsubsection{A $\beta$ 1-40 and A $\beta$ 1-42 ELISA}

Tissues were collected and processed using mouse amyloid $\beta 40$ ELISA kit and amyloid $\beta 42$ ELISA kit (Invitrogen, Inc.) following the manufacture's protocol, with additional guanidine $\mathrm{HCl}$ to extract insoluble amyloid from tissues. Hippocampal tissue was quickly removed on the ice plate. Tissue was kept frozen at $-80 \mathrm{c}$ if not used immediately. Tissue was then homogenized in extraction buffer (with or without additional guanidine $\mathrm{HCl}$ ) and was place at $4 \mathrm{c}$ for $3 \mathrm{~h}$. A protease inhibitor cocktail with AEBSF was added to inactive serine protease. The homogenate was centrifuged at 13,200 $\mathrm{rpm}$ at $4{ }^{\circ} \mathrm{C}$ for $15 \mathrm{~min}$, and the supernatant was collected for further analysis according to instructions.

\subsubsection{Liquid Chromatography-mass Spectrometry (LC-MS)}

Sample preparation. Brain tissues were collected at 1, 2, 6, and 24 hours following i.p. administration of the compound. The brain samples ( $250 \mathrm{mg} / \mathrm{mouse})$ were collected and homogenized after the addition of $100 \mu \mathrm{l}$ de-ionized water. The compounds in the brain homogenates were extracted with $0.9 \mathrm{ml}$ acetonitrile and cooled on ice for 20 min. The supernatants $(700 \mu 1)$ were transferred to a new vial, centrifuged, and dried under N2. The dry samples were reconstituted into $140 \mu 1$ of acetonitrile, mixed thoroughly, and centrifuged, the supernatant were used for LC-MS/MS analysis. Analytical method. LC-MS/MS analysis was performed on an API-4500 triplequadrupole system (AB Sciex, Foster City, CA) with a Turbo V ESI ion source. The 
Table 2-1 List of Primers.

\begin{tabular}{|c|c|c|}
\hline Gene name (coding protein) & Forward/Reverse & Sequences $\left(5^{\prime} \rightarrow 3^{\prime}\right)$ \\
\hline \multirow[t]{2}{*}{ DLG4 (PSD95) } & + & gggacagcetttcctactacc \\
\hline & - & agatctgcgcaaaagtcctg \\
\hline \multirow[t]{2}{*}{ SYN1 (Syn I) } & + & gctgaagcagcagacctga \\
\hline & - & ttcactggtatgaatcactgctg \\
\hline \multirow[t]{2}{*}{ SYP (Synaptophysin) } & + & cactctgtgcccaagtatgg \\
\hline & - & gctgtaggctggagatggag \\
\hline \multirow[t]{2}{*}{$B D N F$} & + & gtcacagcggcagataa \\
\hline & - & gaccttttcaaggactgtgacc \\
\hline \multirow[t]{2}{*}{$G A P D H$} & + & gcaaattcaacggcacag \\
\hline & - & ctcgctcctggaagatgg \\
\hline
\end{tabular}

Notes: List of primers used for qRT-PCR. All primers are listed from 5' to 3'. 
spraying needle voltage was set as $5.5 \mathrm{kV}$ for positive mode. Curtain gas was set at $10 \mathrm{psi}$, drying gas was set to 20 psi, nebulizing gas was set at 20 psi, and CAD gas was set at 7 psi. The probe heater temperature was set at $400^{\circ} \mathrm{C}$. Data acquisition and quantitative processing were accomplished by the Analyst software, version 1.6.1 (AB Sciex, Foster City, CA). Sample solution ( $5 \mu \mathrm{l})$ was injected into a Shimadzu (Canby, OR) Nexera UPLC system. Multiple reaction monitoring (MRM) mode, scanning $\mathrm{m} / \mathrm{z} 457.0 \rightarrow 194.1$ (positive mode), was used to obtain the most sensitive signals. Chromatographic separation of the analytes was performed using a Luna PFP column $(50 \times 2.0 \mathrm{~mm}$ i.d, 3.0 $\mu \mathrm{m}$ particle size, Phenomenex, Torrance, CA) with a guard column, applying gradient elution. Mobile phase A: $0.01 \%$ formic acid in water; B: $0.01 \%$ formic acid in methanol. 0-0.5 min: 2\% B, 0.5-0.6 min: 2-98\% B; 0.6-4 min: 98\% B; 4-4.3 min: 98-2\% B; 4.3-5.0 $\min : 2 \% \mathrm{~B}$. The flow rate was set to $0.3 \mathrm{ml} / \mathrm{min}$.

\subsection{Materials}

\subsubsection{Reagents}

OS47720 was gifted from Oncosynerge. Inc via collaboration. KRIBB11 was purchased from EMD Millipore. Hematoxylin and eosin, 17-AAG, Rp-cAMPs, H89, U0216, KN92, KN93 were purchased from Sigma-Aldrich. Geldanamycin (GA), 17DMAG, LY294002 were purchased from LC labs.

\subsubsection{Antibodies}

17-AAG and antibody against $\beta$-actin were from Sigma-Aldrich. Antibodies against HSF1, Hsp90, Hsp70, Hsp40, Hsp27, PSD95, synapsin I, AKT, p-AKT, Rab 3a, SNAP25, SAP97, and CaMKII were from Cell Signaling Technology. BDNF antibody was from Santa Cruz Biotech. Alexa Fluor-conjugated antibodies were from Invitrogen. Antibody against synaptophysin was purchased from EMD Millipore. Goat anti-Rabbit IgG secondary antibody, Alexa Fluor ${ }^{\circledR} 594$ conjugate and anti-mouse IgG sencondary antibody, Alexa Fluor ${ }^{\circledR} 488$ conjugate were purchased from Thermofisher Scientific.

\subsubsection{Viruses}

AAVH-BH (AAV1/H-BH) overexpressing constitutive form of HSF1 (6.11x1012 viral genomes $/ \mathrm{mL}$ ) and control AAVYFP (AAV1/YFP; $2.64 \times 1012 \mathrm{vg} / \mathrm{ml}$ ) was gifted from Dr. Deborah Young, The University of Auckland, Auckland, New Zealand. Construct of shRNAhsfl was designed by Dr. Yueqiang Xue in Dr. Liao's lab using the following sequence 5'-GCCCAAGTACTTCAAGCACAA-3'. AAVshRNAhsf1 (AAV2/5CMVeGFPhU6shRNAhsf1) was packaged at Viral Vector Core Facility, University of Iowa at the titers of $1 \times 1012 \mathrm{vg} / \mathrm{ml}$. 


\subsection{Statistics}

All statistical analysis was performed by Student's t-test for two groups' comparison, or one way ANOVA followed by Tukey's post hoc test for three or more groups' comparison. Data are presented by means \pm SEM. The asterisks indicate significant difference versus control as follows: $* \mathrm{P}<0.05 ; * * \mathrm{P}<0.01$; and $* * * \mathrm{P}<0.001$. 


\section{CHAPTER 3. 17-AAG'S NEUROPROTECTION AGAINST A $\beta$ TOXICITY}

\subsection{Introduction}

17-AAG is an immediate GA derivative that has been widely investigated for its potential therapeutic effect on various cancers by targeting Hsp90 complex inhibition. It is the first-in-class Hsp90 inhibitor selected by the Cancer Therapy Evaluation Program (CTEP) of the National Cancer Institute (NCI) for clinic studies in cancer. As HSP90 inhibitors, GA and its derivative analog 17-AAG initiate a heat shock-like response and the response was found in multiple cell types, among which most of them were cell lines. Investigations on 17-AAG provided a proof of concept of targeting Hsp90 inhibition in cancer treatment (Gorska et al., 2012; Jhaveri and Modi, 2012). Several clinic trials had been conducted to determine its safety profile and dosing strategy. Phase I trial of 17AAG in patients with advanced cancer (mainly breast and prostate cancers) revealed schedule-dependent toxicity and suggested an intermittent dosing strategy (Goetz et al., 2005). Pharmacokinetic and pharmacodynamic studies of $17-\mathrm{AAG}$ demonstrated the dose level $450 \mathrm{mg} / \mathrm{m} 2 /$ week or $40 \mathrm{mg} / \mathrm{m} 2$ daily for 5 days every 3 weeks had desirable clinic activity (Banerji et al., 2005; Grem et al., 2005). A phase II trial in patients with prostate cancer showed 17-AAG had no activity with regard to the prostate-specific antigen (PSA) response but with a severe grade 3 fatigue (Heath et al., 2008).

Besides the most studied area targeting cancer disease, 17-AAG also exhibited a great potential in treating neurodegeneration. In 2005, 17-AAG was discovered to be neuroprotective in ameliorating polyglutamine-mediated motor neuron degeneration. In cultured SH-SY5Y cells, treatment with 17-AAG induced expression of heat shock proteins. Intraperitoneal injection of 17-AAG was found to degrade polyglutamineexpanded mutant Androgen Receptor (AR), which is a pathogenic gene product in spinal and bulbar muscular atrophy (SBMA), associated with Hsp70 induction in the spinal cord. 17-AAG ameliorated phenotypic expression on SBMA mice (Waza et al., 2005; Waza et al., 2006). In tau pathology, Dou et al. showed that GA and 17-AAG reduced tau phosphorylation and its abnormal aggregation through inhibition of the Raf-MEK-ERK pathway (Dou et al., 2005). Luo et al. revealed the amelioration in AD mice model (JNPL3 line) with tau pathology improvement after 17-AAG treatment (Luo et al., 2007). In a recent comparison study, treatment of 17-AAG significantly reduced NFT area in JNPL3 mice, but not A $\beta$ plaques in Tg2576 mice by thrice weekly intraperitoneal injections (5 and $25 \mathrm{mg} / \mathrm{kg}$ ) for 3 months (Ho et al., 2013). Though the result is not convincing enough due to the known limited CNS-permeability of 17-AAG, it suggest the great potential of 17-AAG in treating AD.

Tg2576 mice have been recognized as a classic mouse model of AD. They overexpress a mutant form of APP (isoform 695) bearing the Swedish mutation (KM670/671NL). Transgenic mice develop A $\beta$ plaques by 12 months. Cognitive function has been extensively characterized in this model, as reported that impaired learning at a spatial task, working memory and progressive impairment at 12 months (Hsiao et al., 
1996; Westerman et al., 2002). In the current study, Tg2576 mice were selected as AD mouse model.

For all the past studies of 17-AAG on neurodegeneration, the major mechanism relied either on inducing heat shock proteins or clearing disease pathology related proteins. In the current study, since AD is also a synapse disease, as a proof of concept, we provided evidence of 17-AAG showing synaptic protection by improving synaptic structure, activity and function.

\subsection{Results}

\subsubsection{7-AAG Induces Rapid and Sustained Expression of HSPs and Synaptic Proteins in Neurons}

In the current study, treatment with $17-\mathrm{AAG}$ was found to rapidly induce HSF1 activation in rat primary cortical neurons (14 DIV), as evidenced by its nuclear translocation (Figure 3-1A). Similar to cancer cells, 17-AAG treatment also led to rapid induction of multiple HSPs in neurons, in particular inducible iHsp70 and Hsp27 (Figure 3-1B), with a sustained effect lasting for at least $24 \mathrm{~h}$ achieved at 100-200 nM concentrations. High concentrations $(>1 \mu \mathrm{M})$ caused cytotoxicity in neurons. Hsp40 expression was also increased, to a lesser degree. No change was found in the constitutive form of Hsp70 (cHsp70). Mechanistically, 17-AAG only disrupts protein interactions between Hsp90 and its clients, therefore it does not change the amount of Hsp90 itself. The activity of the Hsp90 inhibitor was further confirmed by its ability to disrupt a major Hsp90 client protein, protein kinase B/AKT in neurons (Figure 3-1B).

Interestingly, using Western blot it was found that 17-AAG caused an increase in the expression of select presynaptic and postsynaptic proteins (synapsin I, synaptophysin, and PSD95), beginning $1 \mathrm{~h}$ after treatment and reaching peaks within $3 \mathrm{~h}$ (Figure 3-1C). This effect appears to be specific to certain synaptic proteins, since the expression of two other PDZ-containing scaffolding proteins, SNAP25 and SAP97, as well as presynaptic Rab3a and CaMKII (the most abundant postsynaptic protein) were not affected by 17 AAG (Figure 3-1C).

To get further evidence of 17-AAG's effect on established proteins localized at synaptic dendrites, cultured rat hippocampal neurons (21 DIV) were used for immunostaining. The soluble $\mathrm{A} \beta$ used was derived from a $\mathrm{CHO}$ cell line (7PA2) which expresses APP carrying the V717F mutation This cell line has been fully characterized by Walsh et al. (Walsh et al., 2002). 7PA2 cells secrete high levels of monomeric and small oligomeric $A \beta$, with no larger insoluble aggregates. Soluble $A \beta$ oligomers have widely been proven to be a toxic species to brain cells (Mattson et al., 2001; Hashimoto et al., 2003; Pagani and Eckert, 2011). Low levels of soluble $\mathrm{A} \beta(\sim 1 \mathrm{nM})$, which is equivalent to concentrations in human AD brains, can rapidly impair the normal morphology of dendritic spine and uncouple dendritic spines from their nerve terminus (Calabrese et al., 2007; Shankar and Walsh, 2009). By using two-fold diluted conditioned media collected 

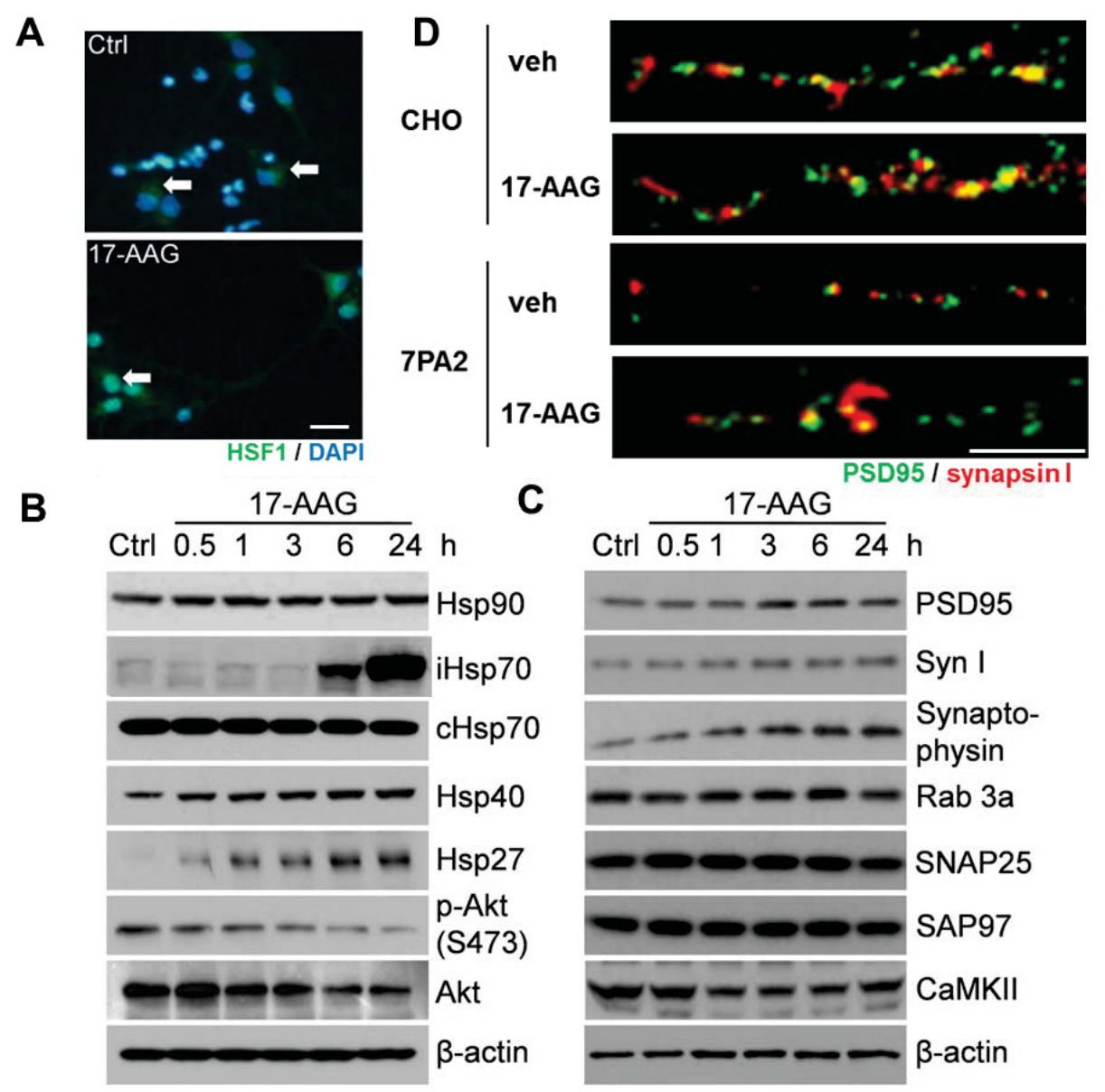

Figure 3-1 17-AAG Induces HSF1 Nuclear Translocation, HSPs and Synaptic Proteins.

Modified with permission. Chen Y, Wang B, Liu D, Li JJ, Xue Y, Sakata K, Zhu LQ, Heldt SA, Xu H, Liao FF. (2014). Hsp90 chaperone inhibitor 17-AAG attenuates A $\beta$ induced synaptic toxicity and memory impairment. J Neurosci. 34, 2464-2470.

(A) Subcellular localization of HSF1 was observed by conducting immunocytochemistry on cultured primary hippocampal neurons (14-21 DIV, $>95 \%$ purity) $0.5 \mathrm{~h}$ after treatment of 200nM 17-AAG, using anti-HSF1 antibody (green) and DAPI (blue). White arrows indicate cytoplasmic HSF1 (green) in the control neurons and overlap between HSF1 signaling (green) with DAPI (blue) in the nuclei in the majority of treated neurons. Representative images are shown from 6 independent experiments with objective magnification set at $25 \times$ during image capturing. Scale bar is $20 \mu \mathrm{m}$. (B) Western blot analysis of HSPs as well as AKT, p-AKT levels after treatment with $200 \mathrm{nM}$ of 17-AAG. (C) Induction of both presynaptic and postsynaptic protein expression by $17-\mathrm{AAG}$, as determined by Western blot analysis. Syn I, synapsin I. Results are representative Western blots of 10 experiments. (D) Immunocytochemistry for PSD95 and synapsin I in mature neurons (21 DIV) $4 \mathrm{~h}$ after exposure to $17-\mathrm{AAG}(200 \mathrm{nM})$ with objective magnification at $60 \times$. Scale bar is $5 \mu \mathrm{m}$. 
from 7PA2 cells (denoted as $A \beta$ ), the desired concentration of soluble $A \beta$ was obtained. In matured neurons (21 DIV), PSD95 and synapsin I protein levels were both significantly reduced at synapses upon soluble $A \beta$ treatment. This was largely restored by 17-AAG co-treatment (Figure 3-1D). Confocal microscopic analysis of the doublelabeled immunofluorescent stained neurons demonstrated that these two vesicle-related antigens were closely colocalized in neuronal dendrites, showing numerous puncta of synapsin I and PSD95 (Figure 3-1D). Consistent with the Western blot data, immunocytochemical results confirmed that 17-AAG not only prevented the A epithelium (OE) $\beta$-induced decrease in individual protein expression, but also rescued functional synapses, as measured by PSD95 clusters and proximate PSD95-synapsin I cluster complexes.

\subsubsection{7-AAG Protects Synaptic Structure Against A}

$A \beta$-induced loss of dendritic spine was further examined, as well as the effect of 17-AAG on spines by the co-treatment of $17-\mathrm{AAG}$ and $\mathrm{A} \beta$, which were determined by quantification of dendritic spine density. Dendritic spines represent the structure in the CNS that allows for multiple inputs and is the highly dynamic site of excitatory synapses. The protective effect of 17-AAG was observed at $24 \mathrm{~h}$ (Figure 3-2A and B). Strikingly, $17-\mathrm{AAG}$ treatment itself slightly increased the number of dendritic spines, and cotreatment significantly improved the $\mathrm{A} \beta$-induced synaptic structure impairment. Because dendritic spines are the primary site of glutamate receptors and synaptic transmission, our findings regarding 17-AAG's potent effect in counteracting the $A \beta$-induced reduction in mature spine density suggest a role for 17-AAG in the functional rescue of $\mathrm{A} \beta$-impaired synaptic transmission. Together with previous results on synaptic protein, these results strongly suggest that 17-AAG induces formation of active synapses which leads to improved synaptic transmission as the clusters of these presynaptic and postsynaptic proteins represent functionally active synaptic zones.

\subsubsection{7-AAG Transcriptionally Upregulates Synaptic Proteins}

Because 17-AAG-induced expression of PSD95 and synapsin I proteins occurs within the first $2 \mathrm{~h}$, the responsible mechanism is more likely through transcriptional activation. In cultured rat hippocampal neurons (21 DIV), qRT-PCR was used to examine mRNA levels of select synaptic proteins after 17-AAG treatment at various time points. Indeed, 17-AAG rapidly increased the mRNA levels of PSD95, synapsin I, and synaptophysin by approximately two-fold, as determined as early as $0.5 \mathrm{~h}$ after treatment by qRT-PCR (Figure 3-3).

\subsubsection{7-AAG Prevents Memory Loss Induced by A}

To test whether 17-AAG can prevent memory loss induced by $A \beta$, an acute $A \beta$ model in adult B6 mice (4-6 months of age) was established, in which 7PA2 cell-secreted 
A

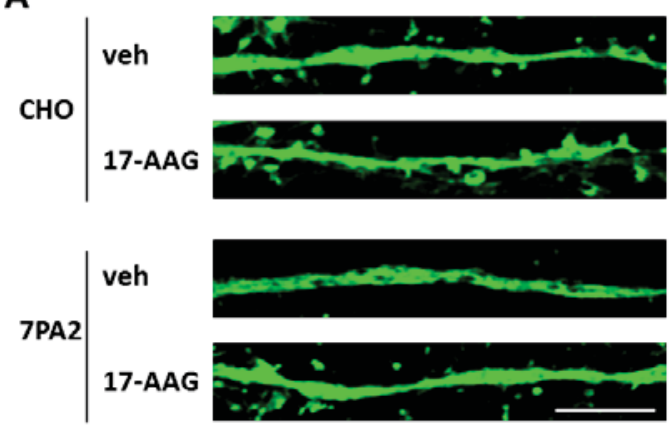

B

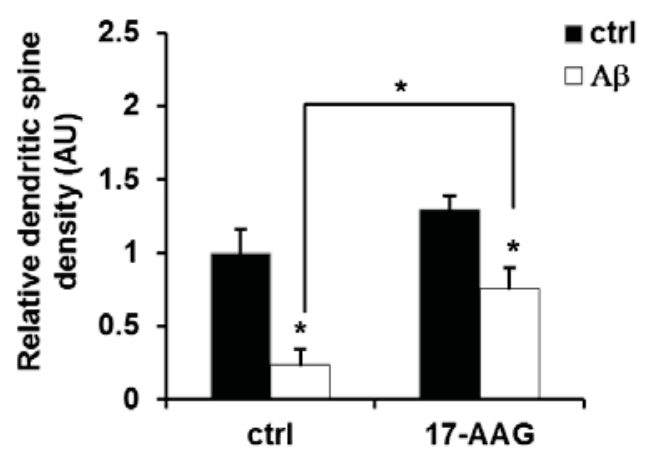

Figure 3-2 17-AAG Protects Neurons from A $\beta$-induced Dendritic Spine Loss. Modified with permission. Chen Y, Wang B, Liu D, Li JJ, Xue Y, Sakata K, Zhu LQ, Heldt SA, Xu H, Liao FF. (2014). Hsp90 chaperone inhibitor 17-AAG attenuates A $\beta$ induced synaptic toxicity and memory impairment. J Neurosci. 34, 2464-2470.

(A) Representative confocal images of dendritic spines on cultured neurons infected with AAV1-YFP for 7 days (7-14 DIV), with indicated treatment for 24 hours. Scale bar is 5 $\mu \mathrm{m}$. (B) Quantification of dendritic spines on 10 fields per slide. N=5. One-way ANOVA was performed followed by Tukey's post hoc test. $* \mathrm{P}<0.05$. Data are represented as mean \pm SEM. 


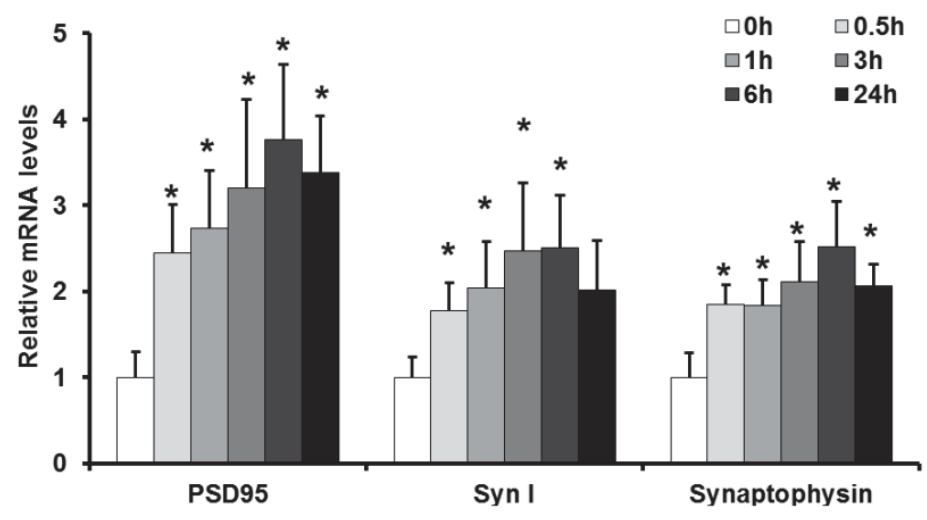

Figure 3-3 17-AAG Activates Transcription of Select Synaptic Genes.

Modified with permission. Chen Y, Wang B, Liu D, Li JJ, Xue Y, Sakata K, Zhu LQ, Heldt SA, Xu H, Liao FF. (2014). Hsp90 chaperone inhibitor 17-AAG attenuates A $\beta$ induced synaptic toxicity and memory impairment. J Neurosci. 34, 2464-2470.

17-AAG upregulates the transcriptional levels of PSD95, synapsin I, and synaptophysin, as determined by qRT-PCR at different time points up to $24 \mathrm{~h}$. ${ }^{*} \mathrm{P}<0.05$. Two-way student's t-test was performed. $\mathrm{N}=3$. Data are represented as mean $\pm \mathrm{SEM}$. 
soluble $A \beta$ oligomers were microinjected into the lateral ventricles using implanted cannulas. Contextual conditioned freezing memory was evaluated by the freezing percentage during the contextual fear conditioning test. A higher freezing percentage reflects better memory. During Day 1, mice received the first injection (injection 1) $1 \mathrm{~h}$ before fear training. The second injection was given $3 \mathrm{~h}$ after the training. On Day 2, a third injection was given $1 \mathrm{~h}$ before the context retrieval test (Figure 3-4A). The contextual fear retention (contextual retrieval) tests were performed from Day 2 to Day 5 at $24 \mathrm{~h}$ interval. These results showed that contextual conditioned freezing memory was impaired by $\mathrm{A} \beta$ injection, but co-injection of 17-AAG $(2 \mu \mathrm{l} / 100 \mu \mathrm{M})$ improved contextual conditioned freezing memory at the $72 \mathrm{~h}$ time point, almost to the level of the control group (Figure 3-4B and $\mathbf{C}$ ). Biochemical analyses were conducted after the behavior test. Both PSD95 and mature Brain-derived neurotrophic factor (BDNF) were completely rescued by co-treatment of $A \beta$ and 17-AAG (Figure 3-4D), according to quantification of Western blot analysis (Figure 3-4E and F).

\subsection{Summary}

In this first part, we discovered that in vitro treatment of 17-AAG promoted HSF 1 nuclear translocation and induced a heat shock-like response in cultured primary neurons, associated with an increase of several select synaptic proteins, such as PSD95, synapsin I, and synaptophysin. This was confirmed by both Western blot analysis and immunofluorescent staining. qRT-PCR showed regulation at the transcriptional level. 17AAG restored expression levels of synaptic proteins reduced by $A \beta$ toxicity and protected neuronal structure by increasing dendritic spines.

Targeting Hsp90 inhibition by inhibitors has yet to be studied using in vivo AD models. The current study further tested 17-AAG by establishing an acute A $\beta$ mouse model and examined memory in contextual fear conditioning test. Stereotaxic microinjection of 17-AAG through implanted cannula rescued memory impairment caused by A $\beta$ toxicity (7PA2 CM). Protein analysis revealed restored PSD95 and BDNF after 17-AAG microinjection. 

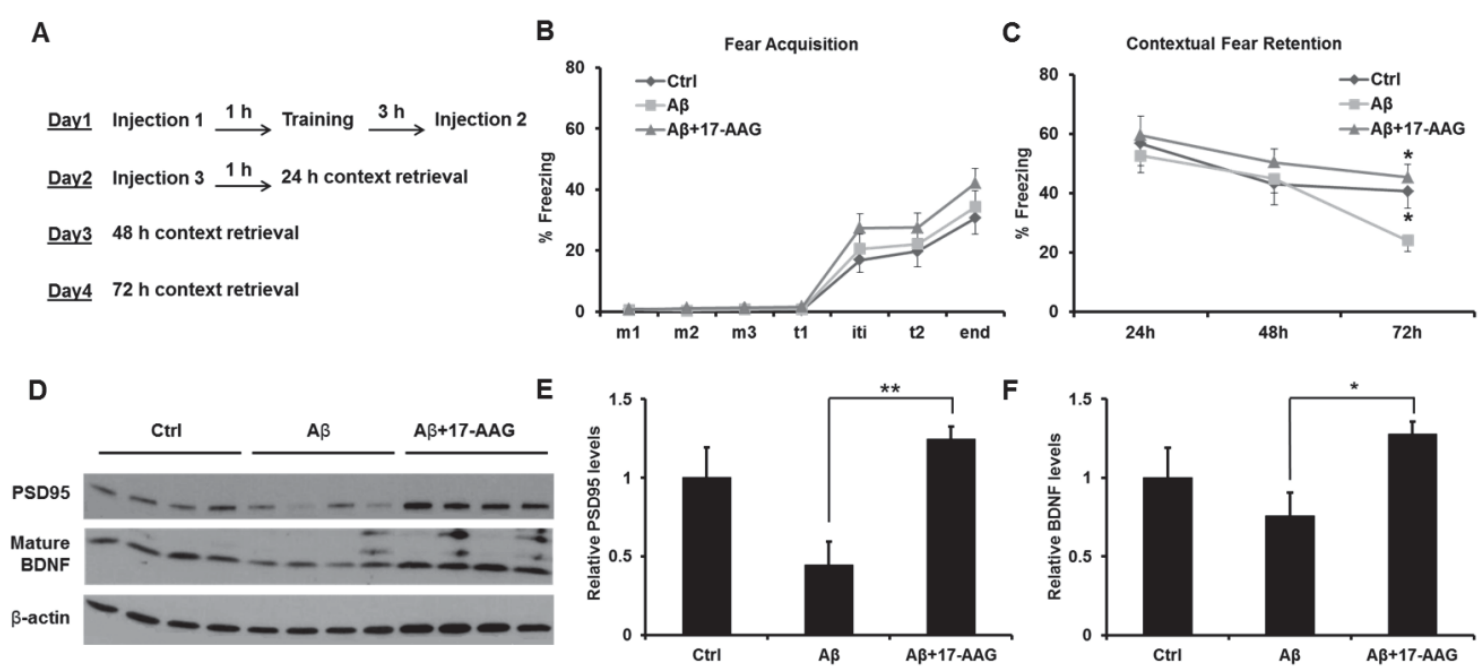

Figure 3-4 17-AAG Prevents Memory Loss Induced by A $\beta$ In vivo.

Modified with permission. Chen Y, Wang B, Liu D, Li JJ, Xue Y, Sakata K, Zhu LQ, Heldt SA, Xu H, Liao FF. (2014). Hsp90 chaperone inhibitor 17-AAG attenuates A $\beta$ induced synaptic toxicity and memory impairment. J Neurosci. 34, 2464-2470.

(A) Experimental design of the contextual fear conditioning assay to test the effect of 17AAG in A $\beta$-induced impairment of contextual conditioned freezing memory. 17-AAG (2 $\mu \mathrm{l} ; 100 \mu \mathrm{M})$ was mixed with $\mathrm{A} \beta$ for the three injections via i.c.v route through implanted cannula. (B) Fear acquisition during the first day training. (C) Recorded contextual fear retention. ${ }^{*} \mathrm{p}<0.05 \mathrm{~N}=14$ animals each group. (D) Protein expression levels of PSD95 and BDNF from the mouse hippocampi after the fear conditioning experiment.

Representative images from Western blot are shown on 4 of 14 mice each group. (E-F) Quantification of the Western blot images by densitometry. One-way ANOVA with Tukey's post hoc test. $* \mathrm{P}<0.05$ and ${ }^{* *} \mathrm{P}<0.01$. $\mathrm{N}=14$ animals each group. Data are represented as mean $\pm \mathrm{SEM}$. 


\section{CHAPTER 4. EVALUATION OF THERAPEUTIC EFFECT OF OS47720}

\subsection{Introduction}

The development of more tolerable Hsp90 inhibitors was promoted by the investigation of potential therapeutics for cancers. Radicicol is a macrocyclic antifungal natural compound isolated from Monocillium Nordinii and Monosporium Bonorden (Delmotte and Delmottte-Plauqe, 1953) with unrelated structure to Geldanamycin (GA), and which competes with GA and specifically binds to the Hsp90 complex at the Nterminus domain. Radicicol represents another category of Hsp90 inhibitors with the feature of non-benzoquinone ansamycine structure. Radicicol does not deplete Hsp90 from cells, but rather promote synthesis as well as the stability of Hsp90, similar to the stress response (Schulte et al., 1998). Radicicol exhibited antitumor activity in vitro, but not in vivo because there are some inherent structural limitations (Soga et al., 2003; Pearl et al., 2008). Later efforts have been prompted to generate new radicicol derivatives due to this unstable structure nature of the compound. Benzoquinone ansamycines and its derivatives were reported to display liver and renal toxicity (Supko et al., 1995; Page et al., 1997), however the radicicol oxime derivative KF25706 had no liver and renal toxicity at the dose effective in tumor inhibition $(100 \mathrm{mg} / \mathrm{kg}$ twice daily for 5 consecutive days) (Soga et al., 1999). Other than KF25706, another novel small molecule series based on radicicol, was designed, synthesized by NexGeneix Pharmaceuticals Inc. (now acquired by Oncosynergy, Inc) in 2009, with the lead compounds of this series being OS47701 (former name NXD30001) and OS47720 (former name NXD30020). OS47701 can cross the blood-brain barrier (BBB) (Zhu et al., 2010), so it can be useful for in vivo efficacy tests. Compared with OS47701, OS47720 appears to be of slightly better potency in induction of heat shock proteins (iHSP70) in cultured primary neurons tested by a labmate in Dr. Liao's lab.

Besides the poor bioavailability of radicicol itself, radicicol derivatives have been synthesized to improve in vivo efficacy (Proisy et al., 2006) in cancer research. Shiotsu et al. tested KF25706 and KF58333 which are both novel oxime derivatives of radicicol. Therapeutic potential for the treatment of chronic myelogenous leukemia (CML) was observed by systemic administration of the compound KF58333 in mice. The underlying mechanism was found to work through the destabilization of Hsp90 client proteins (BcrAbl) (Soga et al., 1999; Shiotsu et al., 2000). More research confirmed the antitumor effect in both in vivo and in vitro (Soga et al., 2001; Soga et al., 2003; Ikuina et al., 2003). However, little or no work has been done in further testing their antitumor effect due to the lack of in vivo efficacy. Until recently, the novel radicicol derivative OS47701 potently inhibited glioblastoma multiforme (GBM) cell proliferation in vitro by degrading many of the key effectors during GBM pathogenesis. In an epidermal growth factor receptor (EGFR) driven genetically engineered mouse model of GBM, OS47701 treatment induced GBM tumor regression and enhanced the general survival rate (Zhu et al., 2010).

Other than cancer, radicicol and its derivatives were also introduced in treating 
neurodegeneration. It was reported that in cultured dorsal root ganglion (DRG) neurons, low concentrations of radicicol promoted neurite outgrowth and neuronal survival of embryonic sensory and sympathetic neurons, but radicicol at higher concentration provided less effect (Sano, 2001). This implied a potential of applying radicicol in neurodegenerative diseases. Radicicol treatment was able to induce a heat shock-like response and delayed aggregation of polyglutamine (polyQ) repeats in organotypic hippocampal cultured slice from R6/2 mice (HD mice model) (Hay et al., 2004). More recently, NXD30001 showed its potency in treating an ALS mouse model with motor neuron degeneration. CNS-permeability was confirmed by the quick distribution to brain and spinal cord. In vivo treatment of NXD30001 in mice prevented aggregation of mutated proteins, fragmentation in mitochondria and neuronal death (Zhu et al., 2010).

In studies discussed in previous chapter, we successfully proved the concept of using an Hsp90 inhibitor in treating AD, mainly through boosting synaptic activity and function. In this chapter, we discuss extensive testing the novel new HS90 inhibitor OS47720 for its effect in both in vitro and in vivo AD models. We examined whether OS47720 can affect synaptic activity and whether long-term OS47720 treatment can prevent $A \beta$ induced memory loss in Tg2576 mice.

\subsection{Results}

\subsubsection{Toxicity Study of OS47720}

To study the toxicity of the OS47720 compound, it was compared to geldanamycin (GA) and the safer compound 17-AAG as the "gold standard" used in numerous clinical cancer trials. The chemical structures of these compounds are shown in Figure 4-1A. GA has long been recognized to have severe hepatotoxicity (Supko et al., 1995; Amin et al., 2005). It was also reported that GA could induce apoptosis in erythrocytes even under physiological concentrations (Jilani et al., 2013). Because of these side effects, several analogues had been developed to reduce systemic toxicity, including 17-AAG and 17-DMAG [17-(2-dimethylaminoethyl)-amino-17desmethoxygeldanamycin]. 17-AAG had been proven to be much less toxic and more stable. These three compounds were selected for comparison of liver and kidney toxicity.

Compounds were injected i.p. at $100 \mathrm{mg} / \mathrm{kg}$ once daily in young adult B6 mouse

$(\mathrm{N}=2)$. Solvent formula was modified based on a publication for NXD30001, OS47720's previous generation (Zhu et al., 2010). Mice from the GA group died within 24h of the injection. This is consistent with all the previous reports (Supko et al., 1995; Amin et al., 2005). During the first toxicity test, one mouse receiving 17-AAG injection was dead at the day 5 after 4 daily injections; another mouse appeared to be normal. Another two mice were used to test the toxicity of $17-\mathrm{AAG}(\mathrm{N}=4$ total). On day 5 , both of these mice were moribund. This indicates that $100 \mathrm{mg} / \mathrm{kg}$ is a sublethal dosage of 17-AAG. In contrast, mice from the OS47720 group were normal in appearance and activity. In order to see cellular changes under the treatments, livers and kidneys were embedded in 


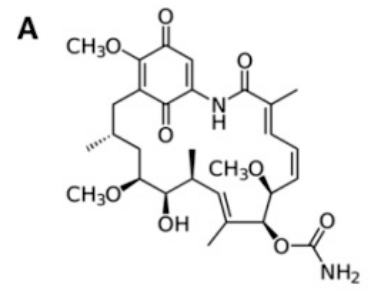

Geldanamycin (GA)<smiles>CC(O)C[C@H](O)/C=C\C=C/C(=O)Cc1c(Cl)c(O)cc(O)c1C(=O)O</smiles>

B

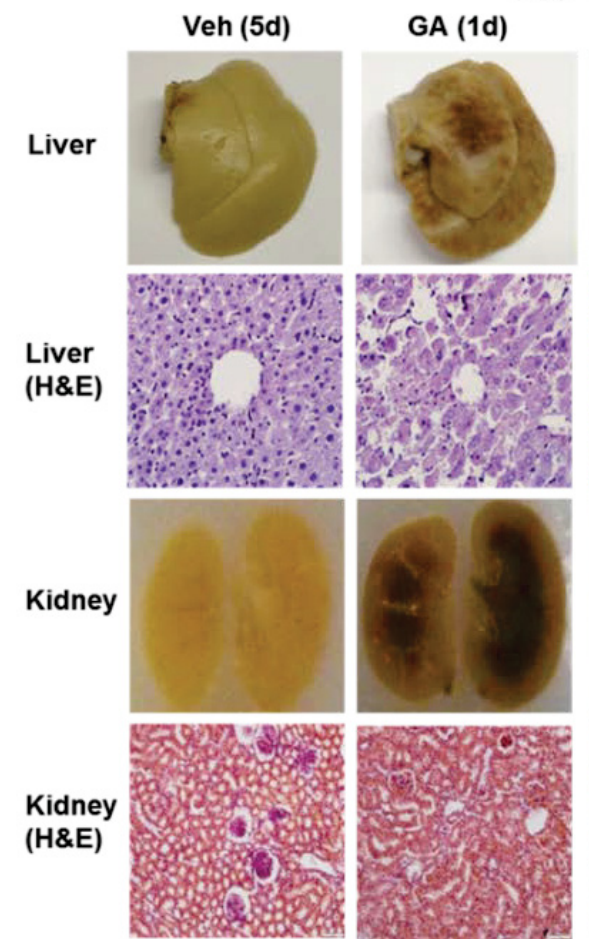

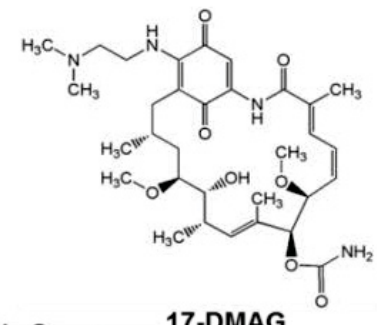

17-DMAG

Pochoxime C (OS47720)

OS47720 (5d) 17-AAG (5d)
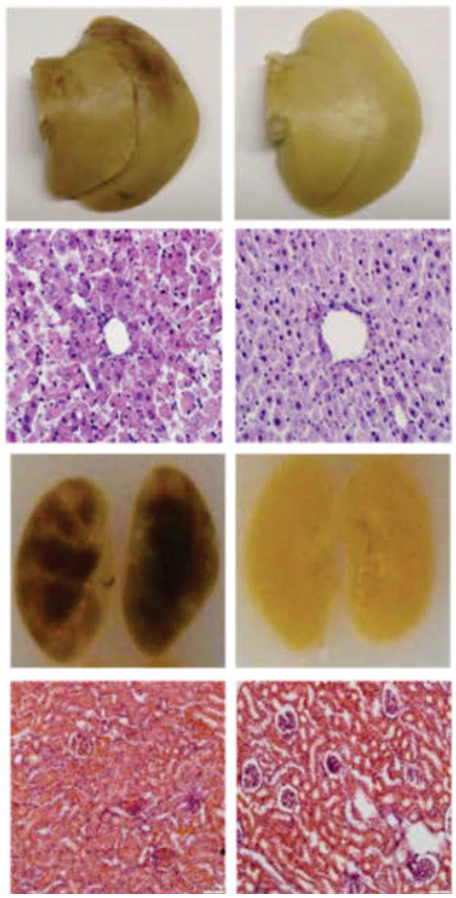

Figure 4-1 Compounds' Structures and Toxicity.

(A) Chemical structures of GA, 17-AAG, 17-DMAG, radicicol and OS47720 (pochoxime C). (B) Representative images of whole liver morphology and H\&E staining (row 1 and 2); representative images of gross kidney morphology in paraffin embedded blocks and H\&E staining. GA and 17-AAG treated mice exhibited dead fragmented hepatocytes (HPCs) and degeneration of the epithelial lining of the renal tubules (row 3 and 4). 
paraffin blocks and hematoxylin and eosin (H\&E) staining was performed.

Hepatotoxicity and nephrotoxicity was evidenced by the presence of dead fragmented hepatocytes (HPCs) and degeneration of the epithelial lining of the renal tubules (representative images shown in Figure 4-1B).

\subsubsection{OS47720 Induces HSPs and Synaptic Proteins in Neurons}

To test if OS47720 compound works similarly as 17-AAG, OS47720 compound was tested in cultured primary hippocampal neurons. Immunofluorescence (IF) staining and confocal imaging revealed that OS47720 induced rapid HSF1 nuclear translocation, an effect which lasted for at least 24h evidenced by the appearance of increased HSF 1 granules (Figure 4-2A). Small $(0.5-1.5 \mu \mathrm{M})$ and large granules $(1.5-3 \mu \mathrm{M})$ were quantified respectively (Figure 4-2B, C and D).

Western blot analysis showed that OS47720 treatment (100 nM) induced iHsp70 (inducible Hsp70) and Hsp40 protein expression (Figure 4-2E and F). Because the Hsp90 inhibitor only disrupted protein interactions between Hsp90 and its clients, it did not change the protein level of Hsp90 itself. Constitutive Hsp70 was also unchanged by OS47720 treatment (Figure 4-2E). OS47720 treatment induced a maximum increase of iHsp70 by $24 \mathrm{~h}$ and the heat shock-like effect lasted for at least $72 \mathrm{~h}$. Besides the induction of iHsp70, other heat shock proteins such as Hsp40 and Hsp27 were also induced, however at somewhat earlier time-points $(0.5 \mathrm{~h}$ and $1 \mathrm{~h}$ respectively). Consistent with the reported 17-AAG data (Chen et al., 2014), synaptic proteins such as PSD95 and BDNF were also upregulated by OS47720 treatment (Figure 4-2F), as further evidenced by quantification analysis (Figure 4-2G). These data demonstrated that OS47720 compound displayed a comparable effect to that of 17-AAG.

\subsubsection{OS47720 Protects Synaptic Structures Against A $\beta$}

Mature hippocampal neurons ( $>21$ DIV) were exposed to soluble A $\beta$ oligomers with and without OS47720 co-treatment. PSD95 (green) and Syn I (red) doublefluorescence staining were performed. Images were captured using a confocal microscope (Figure 4-3A). Consistent with the Western blot analysis data shown in Figure 4-2E and F, OS47720 treatment significantly increased expression of PSD95. Both presynaptic Syn I and postsynaptic PSD95 were greatly impaired by A $\beta$ treatment. This impairment was partially rescued by the co-treatment with OS47720. PSD95 and Syn I clusters were also greatly impaired by $A \beta$ treatment and significantly rescued by OS47720 (Figure 4-3B, $\mathbf{C}$ and $\mathbf{D}$ ).

To identify basic dendrites of neurons, AAV1-YFP was used to highlight neurons so neuronal dendrites could be visualized under a fluorescence microscope. Primary and secondary dendrites were captured and analyzed. Figure 4-3E shows the representative images of primary dendrites. Dendritic spines were promoted by OS47720 treatment alone and greatly impaired by A $\beta$; OS47720 treatment significantly rescued dendrite 

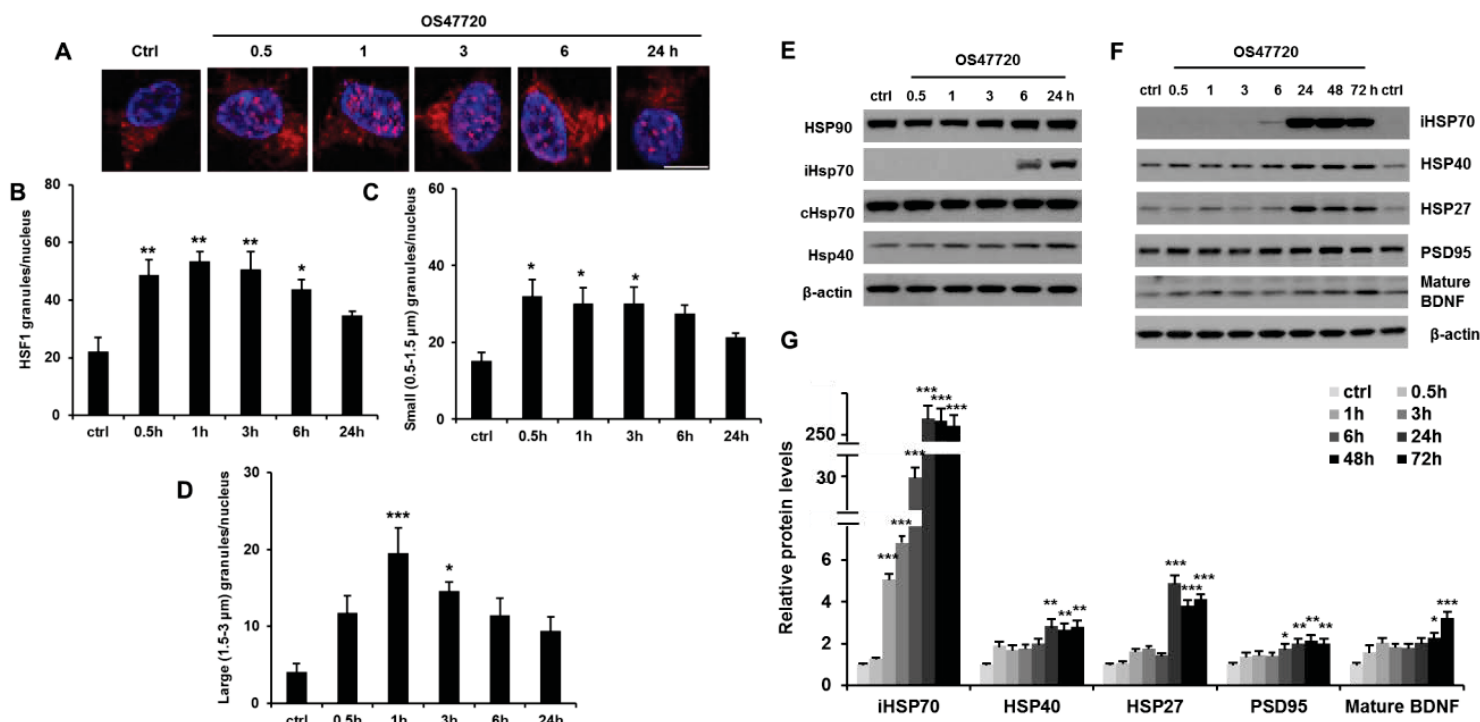

Figure 4-2 Nuclear Translocation of HSF1 Induced by OS47720 in Culture Mature Neurons.

(A) Subcellular localization of HSF1 was observed by conducting immunocytochemistry on cultured primary hippocampal neurons (21 DIV), using anti-HSF1 antibody (red) and DAPI (blue) in a time-course study. Scale bar is $10 \mu \mathrm{m}$. Histograms shows quantification of total (B), small (0.5-1.5 $\mu \mathrm{M}$ in diameter) (C) and large (1.5-3 $\mu \mathrm{M}$ in diameter) (D) granules per nucleus based on 6 9 randomly picked fields per slide from each treatment. $\mathrm{N}=4$. One-way ANOVA was performed followed by Tukey's post hoc test. All time points vs ctrl. $* \mathrm{P}<0.05, * * \mathrm{P}<0.01$ and $* * * \mathrm{P}<0.001$. (E-G) OS47720-induced HSPs and synaptic proteins: (E) Induction of HSPs on Western blot analysis by the treatment of $100 \mathrm{nM}$ OS47720 for 24 hours. (F) Induction of HSPs and synaptic proteins by $100 \mathrm{nM}$ of OS47720 treatment for up to 72 hours. (G) Statistical analysis of Western blot data compared with $\beta$-actin was based on 4 independent experiments. $N=4$. One-way ANOVA with Tukey's post hoc test. All time points vs ctrl. $* \mathrm{P}<0.05, * * \mathrm{P}<0.01$ and $* * * \mathrm{P}<$ 0.001 . Data are represented as mean \pm SEM. 
A

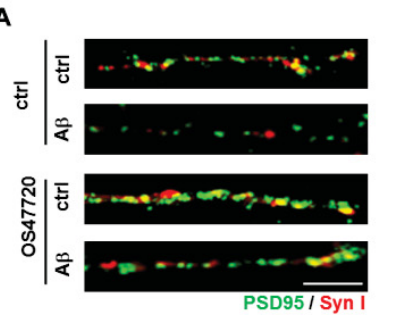

D

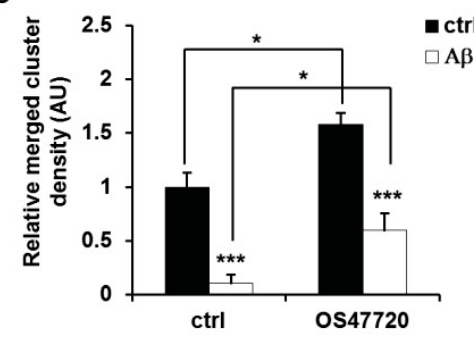

B

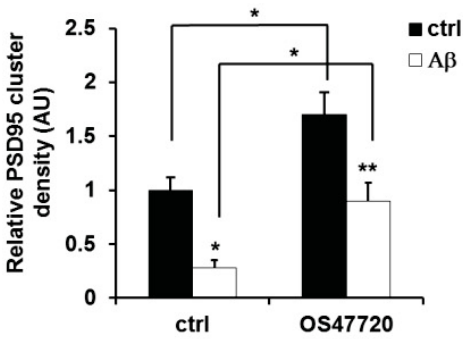

E

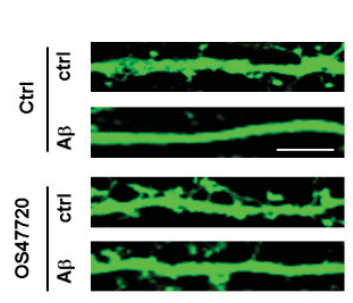

C

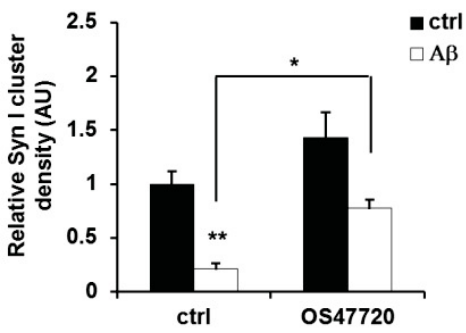

$\mathbf{F}$

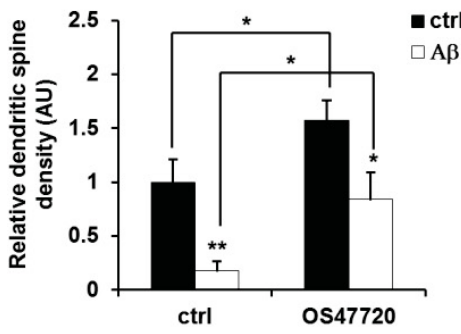

Figure 4-3 OS47720 Exhibits Synaptic Protection Against A $\beta$ Toxicity. (A) Representative confocal images of immunofluorescence labeling of PSD95 and synapsin I, with indicated treatment for 24 hours. Scale bar is $5 \mu \mathrm{m}$. (B-D) Quantification of PSD95 and synapsin I clusters, as well as PSD95/Syn I merged clusters. N=4. (E) Representative confocal images of dendritic spines on cultured neurons transfected with AAV1-YFP for 7 days, with indicated treatment for 24 hours. Scale bar is $5 \mu \mathrm{m}$. (F) Quantification of dendritic spines on 10 fields per slide. $\mathrm{N}=5$. One-way ANOVA with Tukey's post hoc test. ${ }^{*} \mathrm{P}<0.05,{ }^{*} \mathrm{P}<0.01$ and ${ }^{* * *} \mathrm{P}<0.001$. Data are represented as mean \pm SEM. 
spines loss by A $\beta$, as evidenced by the quantification shown in Figure 4-3F.

\subsubsection{Pharmacokinetics of OS47720}

As OS47720 compound is a new compound, there is no literature reporting its basic Pharmacokinetics and Pharmacodynamics, except the recent publication on its previous generation compound NXD30001 (Zhu et al., 2010). By using liquid chromatography-mass spectrometry (LC-MS), a single injection of OS47720 compound in young adult B6 mice at a dosage of $100 \mathrm{mg} / \mathrm{kg}$ induced rapid brain accumulation within $1 \mathrm{~h}$. Then the compound was gradually cleared from $2 \mathrm{~h}$ to $24 \mathrm{~h}$. At $24 \mathrm{~h}$, there is only a trace amount of OS47720 compound remaining in the brain. This data confirmed its CNS-permeability (Table 4-1) and is consistent with the published data on OS47701 (Zhu et al., 2010).

\subsubsection{Pharmacodynamics of OS47720}

Mice received a single i.p. injection of OS47720 at $100 \mathrm{mg} / \mathrm{kg}$. Four tissue samples from the liver, skeletal muscle, spinal cord (lumbar section) and hippocampus (brain) were collected at different time points from $0.5 \mathrm{~h}$ to $48 \mathrm{~h}$. The protein levels of iHsp70 and PSD95 were examined. The liver showed the greatest response to OS47720 compound, as evidenced by the maximum increase of iHsp 70 starting from $2 \mathrm{~h}$ (Figure 4-4A). The iHsp70 protein was most abundant in skeletal muscle, where the heat shocklike response started as early as $0.5 \mathrm{~h}$ after injection. There was no PSD95 protein expression in these two tissues. The hippocampus, the most relevant tissue to memory, also exhibited a good response to OS47720 compound, with iHsp70 starting to increase at $6 \mathrm{~h}$ and lasting for at least 48h. Less iHsp70 was detected in the lumbar spinal cord; however PSD95 levels in both tissues were significantly upregulated starting from $2 \mathrm{~h}$, particularly in the hippocampus where PSD95 is more enriched (Figure 4-4A). This is consistent with the finding that the hippocampus, the memory related region, was one of the main targets in brain.

In addition to Western blot analysis, IF staining was conducted on the collected brain slices. Confocal imaging was performed using Z-scans at a $1 \mu \mathrm{m}$ depth. Nuclear HSF1 (red) was mostly increased in the CA1 region as evidenced by increased fluorescence intensity, to a lesser extent in the CA3 region (Figure 4-4B), but not observed in other brain regions such as cerebral cortex. It is not known if the nuclear HSF1 represents transcriptionally active molecules in neurons.

In order to further investigate the heat shock-like response induced by the OS47720 compound, Hsp70-mPlum reporter mice were used, via collaboration with Dr. Anna M. Planas from Institute for Biomedical Research of Barcelona (de la Rosa et al., 2013). mPlum transgenic mice are generated from an injection of an Hsp70-mPlum farred fluorescence vector with a genomic DNA sequence of the heat shock $70 \mathrm{kDa}$ Protein 1B (Hspa1b) promoter into blastocyst. Young reporter mice received OS47720 at 
Table 4-1 Pharmacokinetics of OS47720.

\begin{tabular}{|c|c|c|c|c|c|c|c|c|c|c|c|}
\hline $\begin{array}{l}\text { Time } \\
\text { point }\end{array}$ & Mouse & $n g / g$ & $\begin{array}{l}\text { Time } \\
\text { point }\end{array}$ & Mouse & $n g / g$ & $\begin{array}{l}\text { Time } \\
\text { point }\end{array}$ & Mouse & $n g / g$ & $\begin{array}{l}\text { Time } \\
\text { point }\end{array}$ & Mouse & $\mathrm{ng} / \mathrm{g}$ \\
\hline \multirow[t]{5}{*}{$1 \mathrm{~h}$} & Mouse1 & 760.5 & $2 \mathrm{~h}$ & Mouse6 & 643.2 & $6 h$ & Mouse9 & 126.9 & $24 h$ & Mouse12 & 4.4 \\
\hline & Mouse2 & 1016.2 & & Mouse7 & 308.2 & & Mouse10 & 92.1 & & Mouse13 & 2.5 \\
\hline & Mouse3 & 1035.5 & & Mouse8 & 290.6 & & Mouse11 & 147.2 & & Mouse14 & 2.7 \\
\hline & Mouse4 & 947.7 & & & & & & & & Mouse15 & 3.1 \\
\hline & Mouse5 & 954.0 & & & & & & & & & \\
\hline $\begin{array}{l}\text { Mean } \\
\pm \\
\text { STD }\end{array}$ & & $\begin{array}{c}942.8 \\
\pm \\
108.8\end{array}$ & & & $\begin{array}{c}414.0 \pm \\
198.7\end{array}$ & & & $\begin{array}{c}122.1 \pm \\
27.9\end{array}$ & & & $\begin{array}{c}3.2 \\
\pm \\
0.9\end{array}$ \\
\hline
\end{tabular}

Notes: OS47720 brain concentrations after single injection of OS47720 at $100 \mathrm{mg} / \mathrm{kg}$ intraperitoneally. Brains were collect at four different time points (1h, 2h, 6h and 24h). N=3-5. 

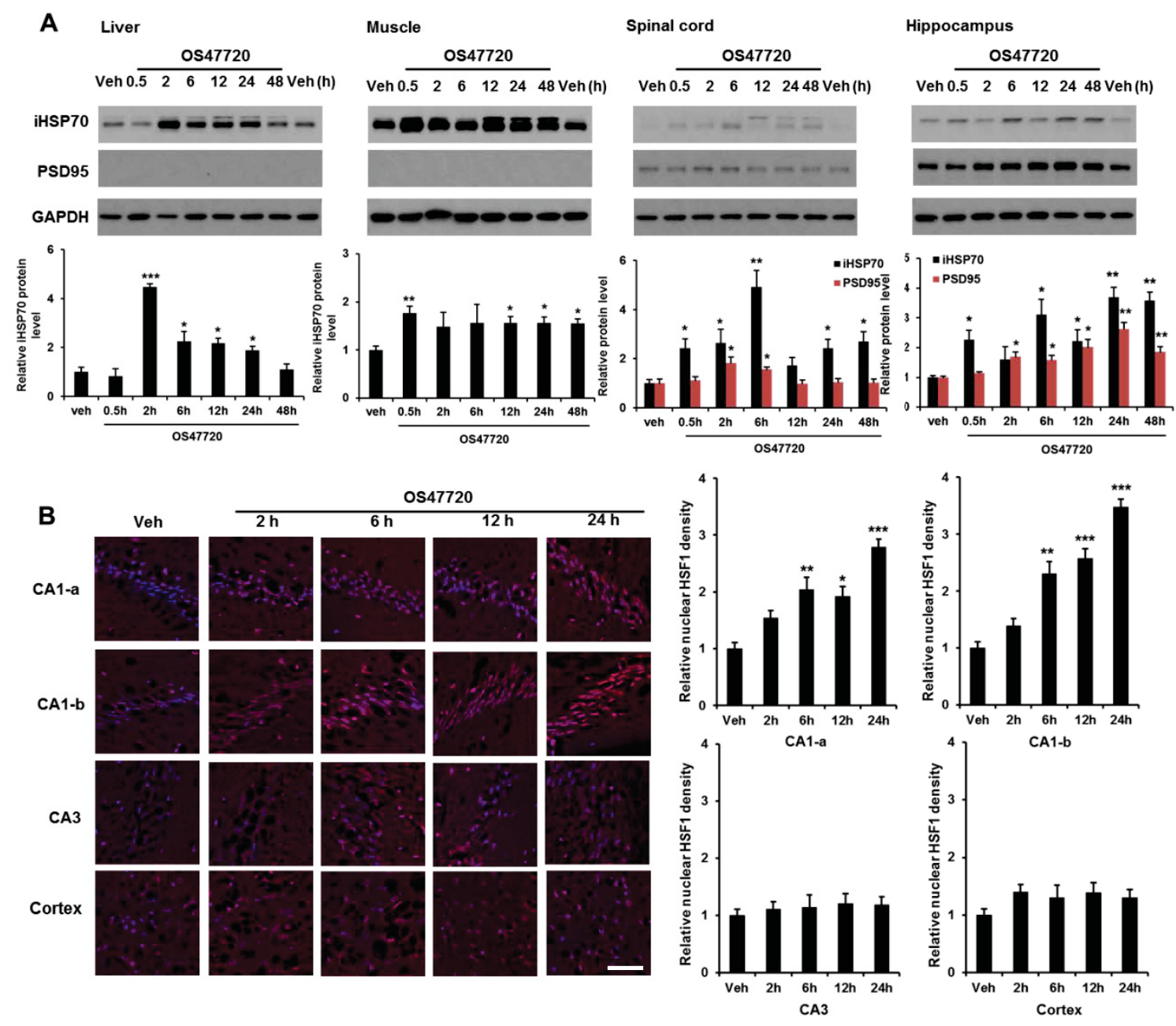

Figure 4-4 Pharmacodynamics of OS47720.

(A) Mouse liver, skeletal muscle, lumbar spinal cord, and hippocampi were collected at 6 different time points after i.p. injection of OS47720 at $100 \mathrm{mg} / \mathrm{kg}$. Western blot analyses detecting inducible Hsp70 (iHsp70), PSD95, and GAPDH were performed. Histogram below shows statistical analysis. $n=3$. (B) Representative images of HSF1 immunohistochemistry (red) of the four different regions and sub-regions of mouse brain (Hippocampal CA1-a, CA1-b, CA3 and cerebral cortex), with nuclei stained with DAPI (blue). Images were captured by a confocal microscope at $600 \times$ magnification. Scale bar is $50 \mu \mathrm{m} . \mathrm{n}=3$. One-way ANOVA was performed followed by Tukey's Post hoc test, all groups vs Veh, ${ }^{*} \mathrm{P}<0.05, * * \mathrm{P}<0.01$ and $* * * \mathrm{P}<0.001$. Data are represented as mean \pm SEM. 
$100 \mathrm{mg} / \mathrm{kg}$ through a single i.p. injection and tissues were collected at several time points following injection. Mice were perfused with 4\% paraformaldehyde (PFA). Brains and livers were nfiltrated with $30 \%$ sucrose for cryoprotection, followed by embedding with OCT embedding. Z-scans were performed on the confocal microscope. Different subregions within hippocampus are presented in Figure 4-5A. Enhanced far red signal was obtained in the CA1 and CA3 regions of mice receiving OS47720, which indicated the increase of $\mathrm{iHsp} 70$, the major target of activated HSF1. Liver showed the strongest response to OS47720 compound (Figure 4-5B), consistent with the protein changes shown by the previous Western blot analysis (Figure 4-4B), although the response was weaker than that in liver. Despite a relatively weak heat shock-like stress response, the induction of synaptic proteins is strong and long-lasting in hippocampi. These findings imply a potentially important role of enhanced HSF1-mediated regulation in hippocampus-mediated functions, such as learning and memory, in specific sub-regions within hippocampus.

In comparison to the more significant increase in nuclear HSF1 levels, iHsp70 induction is relatively weak in hippocampal neurons compared to the liver. This indicates a weak heat shock like stress response in neurons when compared to other tissues, which can explain the particular vulnerability of neurons during neurodegeneration.

\subsubsection{OS47720 Rescues Contextual Conditioned Freezing Memory Loss Induced by $\mathbf{A} \boldsymbol{\beta}$}

In order to test whether OS47720 could protect synaptic function and prevent memory loss in mice induced by $A \beta$, we used the same experimental design for this acute model as in 17-AAG's study in previous chapter was used (Figure 4-6A). In young C57BL/6 mice, A $\beta$ (from 7PA2 conditioned medium [Chen et al., 2014]) was administered 5 days after daily OS47720 injection (ctrl group received vehicle injection), three times via i.c.v. injection through the implanted cannula at the indicated time points (Figure 4-6A). The acquisition of freezing behavior was recorded during the first day of training, and the contextual fear retention was recorded 24, 48 and $72 \mathrm{~h}$ later. As shown in Figure 4-6B, co-treatment of OS47720 significantly rescued contextual conditioned freezing memory impaired by $A \beta$, most significantly at the $72 \mathrm{~h}$ time point. The protein levels of both PSD95 and BDNF were restored to the control level (Figure 4-6C and D). Fear acquisition was unaffected by $\mathrm{A} \beta$.

\subsubsection{Chronic OS47720 Treatment Did Not Change Either Anxiety or Locomotor Activity}

OS47720 (100 mg/kg i.p. every two days for 3 months) was firstly tested on presymptomatic (early-stage) Tg2576 and littermate control mice starting from 9 months of age to 12 months of age. This dosage was determined based on a previous study (Zhu et al., 2010) and previous experiment in which the heat shock effect lasted for at least 48hafter a single injection of OS47720, based on the PD data. Mice were divided into the 


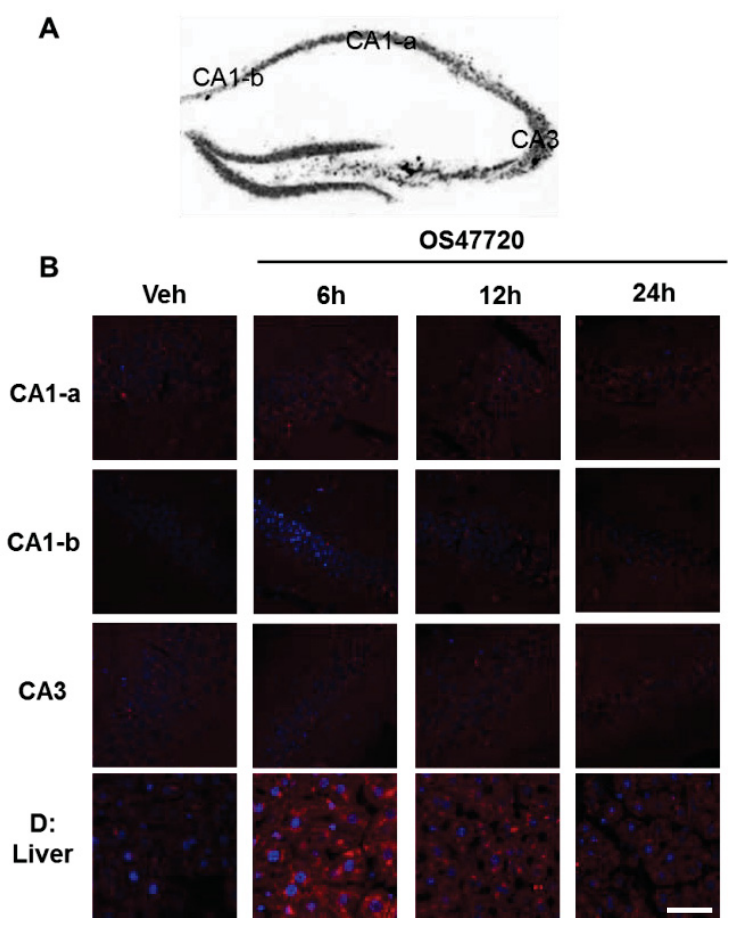

Figure 4-5 OS47720 Induces Hsp70 Protein Expression on Reporter Mice.

(A) Diagram indicates sub-regions of hippocampus. (B) mPlum-Hsp70 reporter mice received OS47720 intraperitoneally at $100 \mathrm{mg} / \mathrm{kg}$. Brains and livers were perfused at different time points, OCT embedded, cryostat-sectioned $(10 \mu \mathrm{M})$, and immunostained with anti-HSF1 antibody (red) and DAPI (blue). Figure shows representative images from confocal microscopy at $600 \mathrm{x}$ magnification. Scale bar is $100 \mu \mathrm{m}$. 


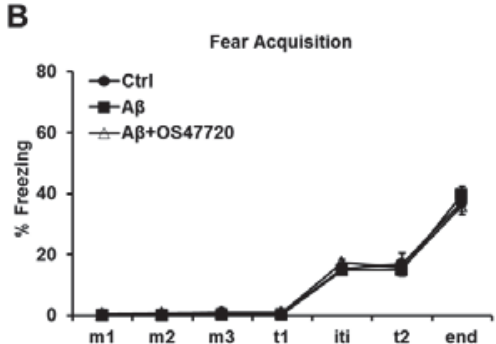

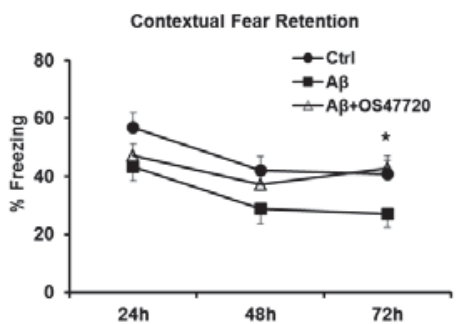

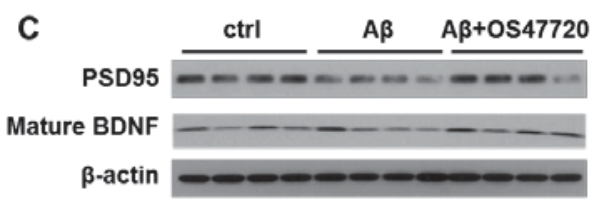

D

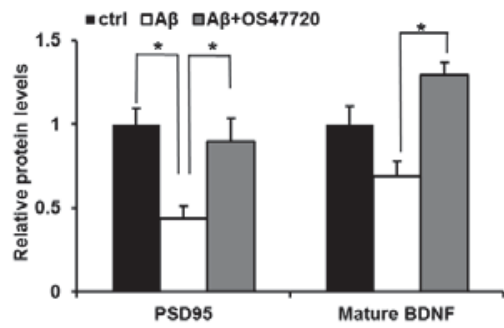

Figure 4-6 OS47720 Prevents Memory Loss Induced by A $\beta$ In vivo.

(A) Experimental design of the contextual fear conditioning test to test the effect of OS47720 against A $\beta$-induced impairment of contextual conditioned freezing memory. (B) Freezing time (\%) during fear acquisition (training) and fear retention (context retrieval) in contextual fear conditioning test. $N=12$. (C) Protein expression levels of PSD95 and mature BDNF from the mouse hippocampi after the experiment. Representative Western Blot images are shown on 4 of 12 mice each group. (D) Quantification of the Western blot images. One-way ANOVA with Tukey's post hoc test. $* \mathrm{P}<0.05$. $\mathrm{N}=12$ animals per group. Data are represented as mean \pm SEM. 
following four groups: 1. NonTg2576-Vehicle group (NonTg-Veh); 2. NonTg2576OS47720 group (NonTg-OS47720); 3. Tg2576-Vehicle group (Tg-Veh); 4. Tg2576OS47720 group (NonTg-OS47720). There were 20 or 21 mice in each group with half males and half females.

To test whether OS47720 affects basal locomotor activity and anxiety, the open field test and elevated plus maze were used. Three parameters were evaluated in the open field including total activity (by beam breaks), distance travelled and time spent in the center. Open field test showed that Tg2576 mice had slightly less locomotor activity than littermate controls, whereas treatment of OS47720 increased locomotor activity slightly (Figure 4-7A, B and C), but these were not statistically significant results.

The elevated plus maze test was conducted to evaluate if OS47720 caused any anxiety-like behavior. The preference for being in open arms over closed arms (expressed as a percentage of time spent in the open arms) is calculated as a measure of anxiety-like behavior. There were no group difference on any measure of anxiety, including total arm entries, arm entries in closed and open arms (Figure 4-7D), and percentage of time spent on center, closed and open arms (Figure 4-7E). According to the analysis based on different sex, there was a small effect of sex on percentage of time spent on open arms such that female mice of all groups spent around 5\% more time on open arms than male mice, and less time in the center. No sex difference in time spent in the closed arms was observed. This small difference should not affect performance on the cognitive tasks, especially given that there are equal numbers of male and female mice in each group $(\mathrm{N}=20$ or 21$)$.

\subsubsection{OS47720 Rescues Working Memory and Spatial and Learning Memory Deficits in Presymptomatic Tg2576 Mice}

All groups of mice were tested for their working memory after chronic OS47720 treatment in a cross maze test. Total entries were recorded for basal activity and alternation rate was calculated and analyzed as the measure of memory. Working memory was greatly impaired in the Tg2576 group, as indicated by significantly lower alternation rate on the cross maze test, and this was almost completely rescued by OS47720 (Figure 4-8A and B).

The Morris water maze test was utilized to measure spatial and learning memory. Experimental schedule is shown in Figure 4-8C. Distance was used as a dependent measure because of a slightly (but non-significant) faster swimming speed in the TgVehicle group, compared to the other three groups. Mice in all groups readily found the cued platform (Figure 4-8D). On the hidden-platform training, there were significant effects of genotype and drug, and a significant Genotype x Drug interaction. Vehicletreated Tg mice were significantly impaired relative to their Non-Tg counterparts (Figure 4-8D), consistent with previous reports in these mice (Westerman et al., 2002). OS47720 treatment rescued the spatial learning impairment in the Tg group, demonstrated by significantly shorter swim paths. OS47720 treatment did not improve spatial learning and 


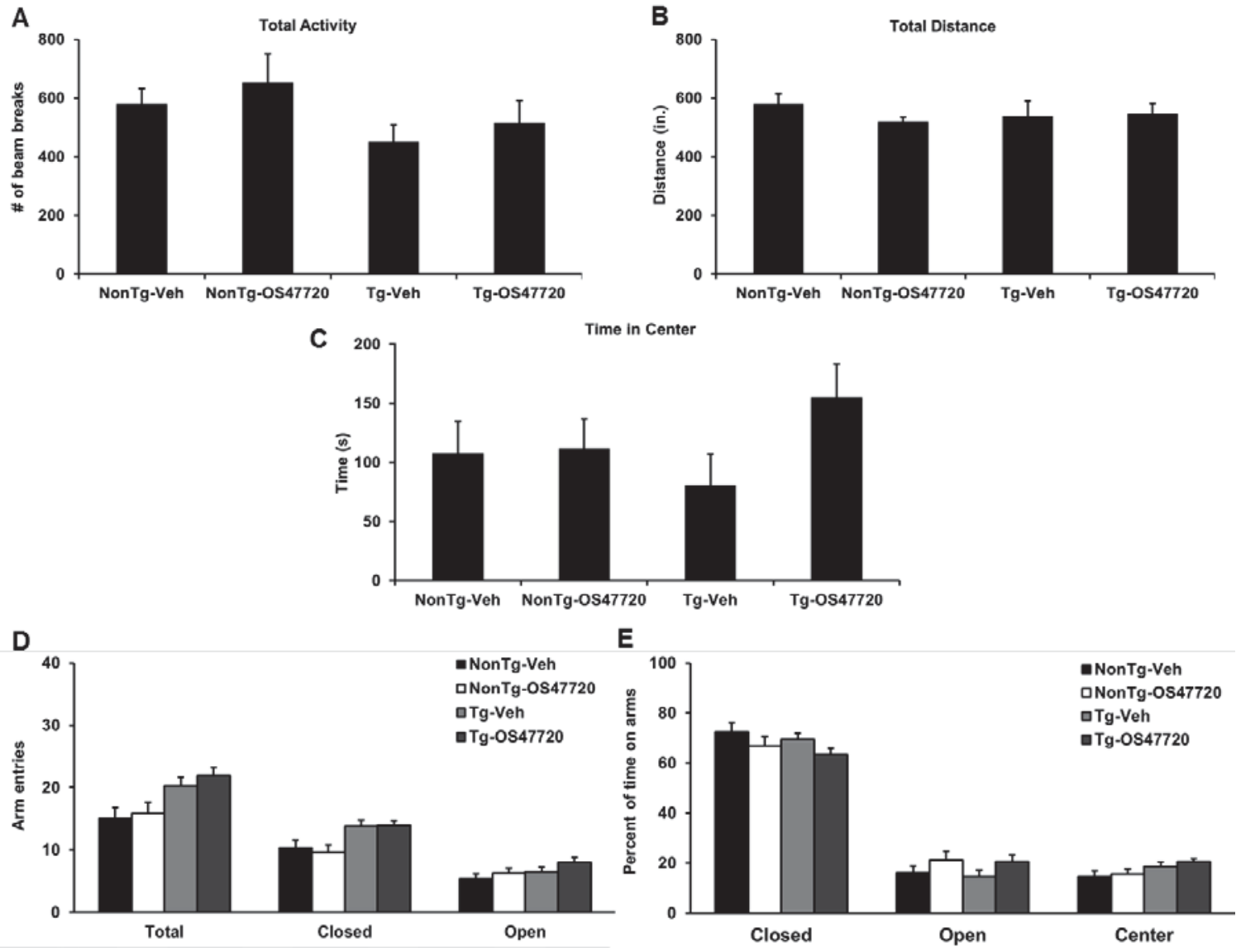

Figure 4-7 Locomotor Activity and Anxiety Level after 3-month Treatment of OS47720.

(A-C) Locomotor activity in 12-month old Tg2576. Locomotor activity was measured by an open field test in all four groups. Numbers of beam breaks, distance travelled (inches), and time (s) spent in the center were measured. (D and E) Anxiety level was measured in the elevated-plus maze. Total arm entries, and percentage of time spent on closed, and open arms and in the central area, were also measured. $\mathrm{N}=20$ or 21 each group. One-way ANOVA was performed. No significant differences were noted. 


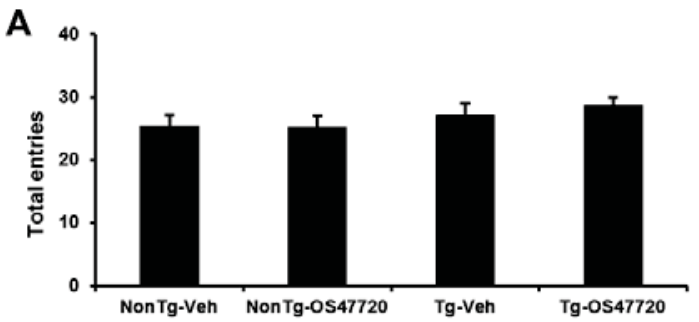

B

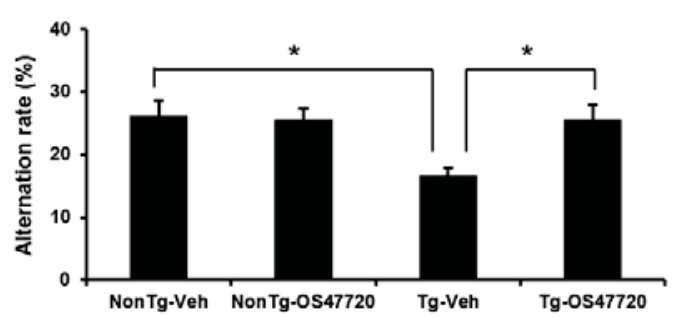

C
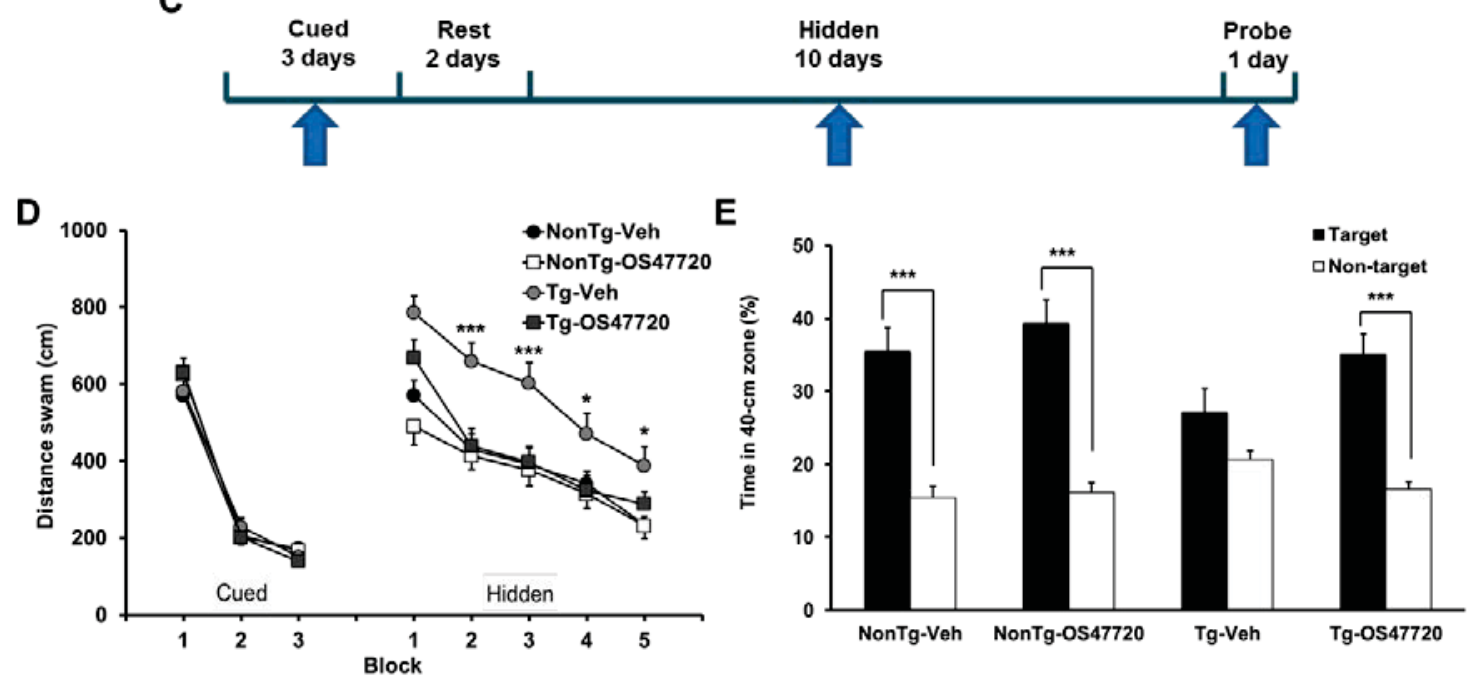

$\mathbf{F}$

G

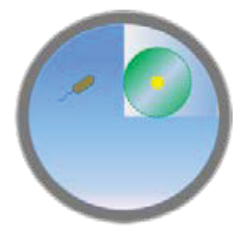

NonTg

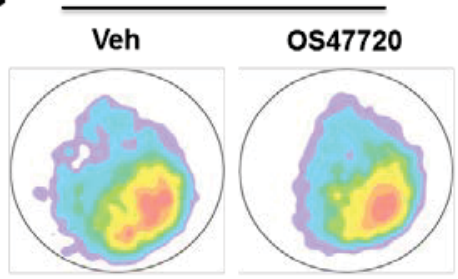

$\mathrm{Tg}$

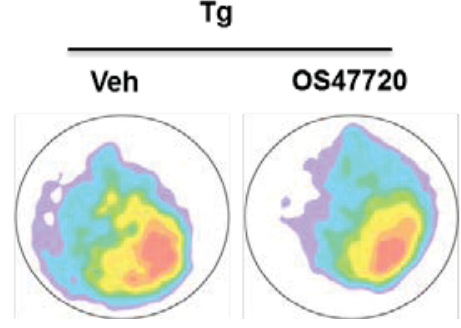

Figure 4-8 In vivo Therapeutic Efficacy of OS47720 in Tg2576 Mice. Tg2576 mice and their littermates were administrated vehicle control or OS47720 treatment $(100 \mathrm{mg} / \mathrm{kg})$ via i.p. for a total of 3 months, starting from 9 months of age. Cross maze and Morris water maze ( $\mathrm{N}=20-21)$ were conducted at 12 months of age. (A) Total entries in cross maze among four experimental groups. (B) Alteration rate (\%) representing working memory on cross maze was calculated based on times of alterations and total entries. (C) Schematic diagram shows schedule of Morris water maze experiment. (D) Distance swam was used in Morris water maze test to evaluate spatial and learning memory. Histogram represents distance swam $(\mathrm{cm})$ cued and hidden platform sessions. Distance was averaged every two days in hidden platform session. (E) Time spent in target $(40 \mathrm{~cm})$ and non-target zones during probe session were analyzed among groups. (F) Schematic diagram shows target zone surrounding previous location of platform and non-target zone along the pool. (G) Representative images of the heat map from each experimental group during the probe session. One-way ANOVA with Tukey's post hoc test. $* \mathrm{P}<0.05, * * * \mathrm{P}<0.001$. Data are represented as mean $\pm \mathrm{SEM}$. 
memory in the non-Tg mice and there was no genotypic difference in the OS47720treated mice (Figure 4-8D). A probe trial was conducted $24 \mathrm{~h}$ after the last hiddenplatform session to assess memory for the former platform location. Time spent in a 40$\mathrm{cm}$ zone centered on the former platform location was used as an index of spatial memory as it was a more precise measure in the target quadrant (Figure 4-8F). The vehicle-treated transgenic mice did not spend significantly more time in the target zone, when compared to the mean of the other three zones (Figure 4-8E). Similarly, these mice did not discriminate between the former platform location and equivalent positions in the other three quadrants. In contrast, mice in the other three groups showed a selective search for the targets. Heat maps show a more defined swim path in OS47720 -treated groups, indicating superior spatial reference memory (Figure 4-8G).

\subsubsection{Protein Changes in Presymptomatic Tg2576 Mice after OS47720 Treatment}

To test for underlying protein changes after chronic treatment. Tissue samples from the hippocampus were harvested from the mice from each group. Western blot analysis was conducted to examine several proteins of interest, including heat shock proteins such as HSF1, iHsp70, Hsp40, synaptic proteins such as PSD95, synapsin I, SNAP25, as well as BDNF.

Consistent with our in vitro data, the expression levels of heat shock proteins including HSF1, iHsp70 and Hsp40 were boosted by OS47720 treatment either in two NonTg2576 groups (NonTg-Veh and NonTg-OS47720), or in two Tg2576 groups (TgVeh and Tg-OS47720) (Figure 4-9A). This indicates the ability of OS47720 to induce a heat shock-like response in the brain through systemic i.p. injection. Moreover, a reduction of heat shock proteins in Tg-Veh group of mice was also observed. In NonTg2576 groups, OS47720 did not significantly upregulate synaptic proteins except for synapsin I and had no effect on BDNF. However, select synaptic proteins, PSD95 and BDNF, were largely restored back to control levels (NonTg-Veh) in the hippocampi of Tg2576 mice after chronic OS47720 treatment (Figure 4-9A). Quantification analysis revealed significant changes occurred in the brain (Figure 4-9B)

\subsubsection{OS47720 Rescues Working Memory and Spatial and Learning Memory Deficits in Symptomatic Tg2576 Mice}

Successful chronic treatment of presymptomatic Tg2576 mice lead to testing repeated OS47720 injections (100 mg/kg i.p.; every 2 days for the first 2 month, and twice a week thereafter until 6 months) as a chronic treatment on symptomatic (middlestage) Tg2576 mice from 12 months to 18 months of age. Mice were divided into the following four groups: 1. NonTg2576 group (NonTg); 2. NonTg2576-Vehicle group (NonTg-Veh); 3. Tg2576-Vehicle group (Tg-Veh); 4. Tg2576-OS47720 group (NonTgOS47720). There were 10-13 mice in each group with about half males and half females. A NonTg2576 group without vehicle treatment was introduced to test if the vehicle itself caused any systemic effect either on body weight or behavior. However, the NonTg- 


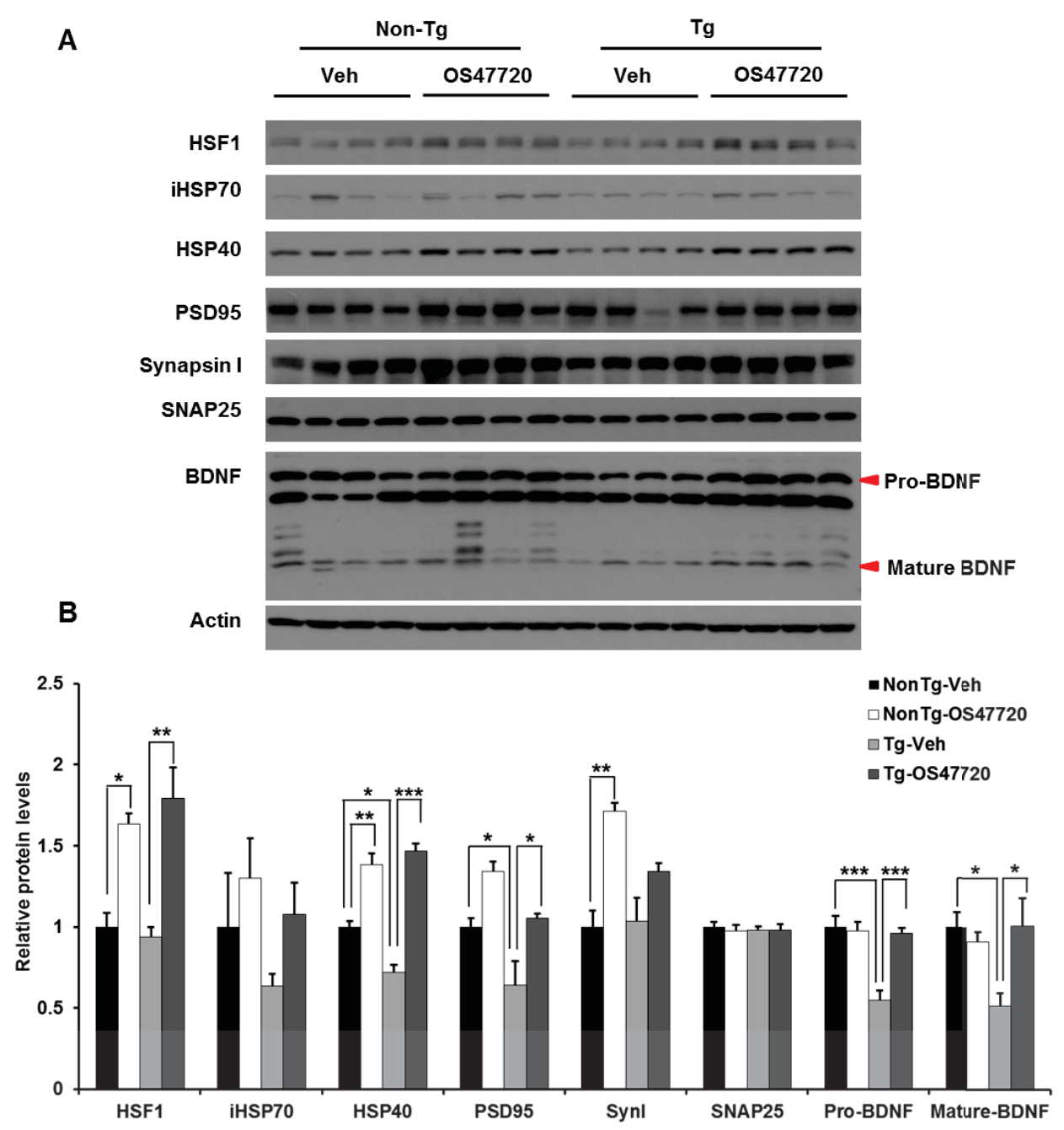

Figure 4-9 End Point Western Blot Analysis after 3-month Treatment of OS47720.

Hippocampal tissues were collected for protein analysis after behavior testes. (A) Representative images (4 mice from each group) of Western blot analysis after Morris water maze. HSF1, Heat shock proteins (iHsp70, and Hsp40), synaptic proteins (PSD95, synapsin I, and SNAP25) and BDNF were tested in four groups; (B) Statistical analysis of the Western blot data presented in (g). N=6. One-way ANOVA with Tukey's post hoc test. $* \mathrm{P}<0.05, * * \mathrm{P}<0.01,{ }^{*} * \mathrm{P}<0.001$. Data are represented as mean $\pm \mathrm{SEM}$. 
OS47720 group was not included this time due to the limited quantity of OS47720 compound. Besides, OS47720 had been proven to induce heat shock-like response and several synaptic proteins in presymptomatic Tg2576 mice. Similar to previous results, the working memory deficit in the Tg-Veh group was significantly improved by OS47720 treatment in $\mathrm{Tg}$ mice, with no difference in total entries among all groups (Figure 4-10A and B). The Morris water maze test revealed impaired long-term spatial learning and memory in the Tg-Veh group, which was significantly improved in the Tg-OS47720 group to the point where memory was comparable to that of the non- $\mathrm{Tg}$ and non-Tg-Veh groups (Figure 4-10C). During probe trial sessions, the Tg-Veh group lacked preference in the target search, while the other three groups selectively searched for the target zone (Figure 4-10D). Vehicle treatment had no effect on cross maze or Morris water maze tests. Taken together, OS47720 exhibited its ability to reverse memory loss in both presymptomatic and symptomatic $\operatorname{Tg} 2576$ mice.

\subsubsection{End Point Study Revealed Protein Changes in Symptomatic Tg2576 Mice after OS47720 Treatment}

Western blot analysis was also conducted to examine changes in select proteins in symptomatic Tg2576 mice after chronic OS47720 treatment. Tissue samples from hippocampus were also collected for biochemistry analysis.

Vehicle treatment induced iHsp70 protein, though not significant, in NonTg2576 mice. However the other two heat shock proteins HSF1 and Hsp40 remained unchanged. Vehicle itself did not change levels of select synaptic proteins (Figure 4-11A). In the Tg2576 groups, Heat shock proteins, PSD95 and BDNF were all reduced in the Tg-Veh group of mice. Chronic treatment of OS47720 reversed not only all heat shock proteins tested, but also synaptic protein PSD95 and BDNF (Figure 4-11A). Quantification analysis demonstrated the significant protection of OS47720 in Tg2576 mice (Figure 4-11B). This result is consistent with the conclusion from previous results of 3 month treatment. In summary, OS47720 sufficiently exhibited its therapeutic potential in preventing memory loss in presymptomatic and symptomatic stages of Tg2576 mice, accompanied by the recovery of impaired heat shock-like response, synaptic proteins and BDNF.

\subsubsection{Body Weight Changes in Presymptomatic and Symptomatic Tg2576 Mice During OS47720 Treatment}

Body weight was not significantly changed during the 3 month chronic treatment of OS47720 treatment in presymptomatic Tg2576 in either males or females (Figure 4-12A and B). There was a slight decrease in body weight (10-14\% on males and $5-12 \%$ on females) over a 6-month treatment course in all vehicle and OS47720 treatment groups, suggesting mild toxicity or stress caused by the vehicle. However, OS47720 treatment itself did not cause obvious weight loss (Figure 4-12C and D). These data confirm the low toxicity of the OS47720 compound. 
A

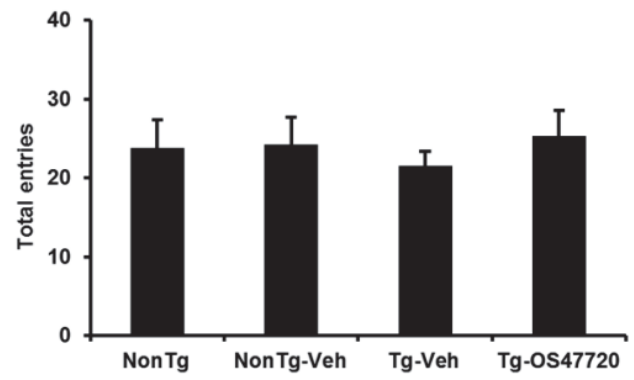

C

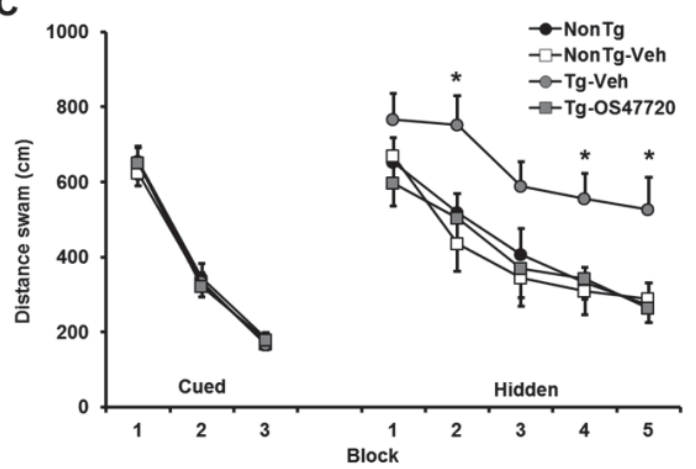

B

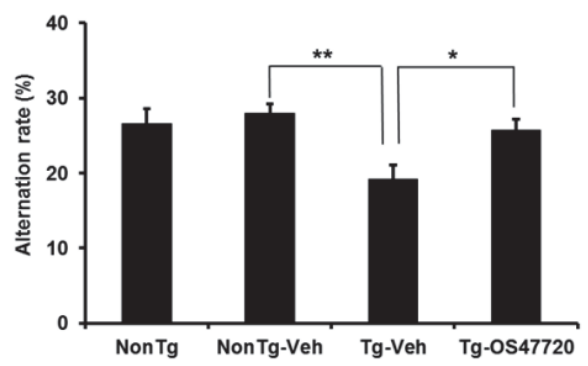

D

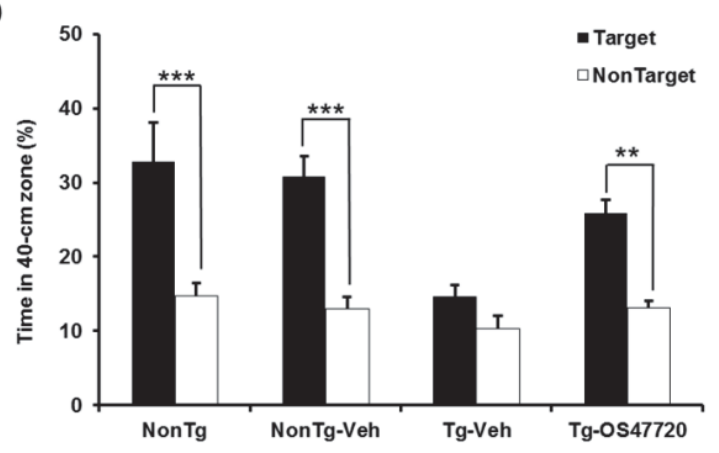

Figure 4-10 Effects on Symptomatic Tg2576 Mice after 6-month Treatment of OS47720.

Each group includes half males and females. (A) Total entries in the cross maze. (B) Alteration rate $(\%)$ representing working memory on the cross maze was calculated as the number of alterations divided by (total entries-3) x100. (C) Distance swam was used in the Morris water maze test to evaluate spatial and learning memory. Histogram represents distance swam $(\mathrm{cm})$ in cued and hidden platform sessions. Distance was averaged every two days in the hidden platform sessions; (D) Time spent in the target $(40 \mathrm{~cm})$ and nontarget zones during probe session was analyzed among groups. $\mathrm{N}=10-13$. One-way ANOVA was performed followed by Tukey's Post hoc test. NonTg-Veh vs NonTgOS47720, Tg-Veh vs NonTg-Veh, Tg-Veh vs Tg-OS47720, ${ }^{*} \mathrm{P}<0.05, * * \mathrm{P}<0.01$ and $* * * \mathrm{P}<0.001$. Data are represented as means $\pm \mathrm{SEM}$. 


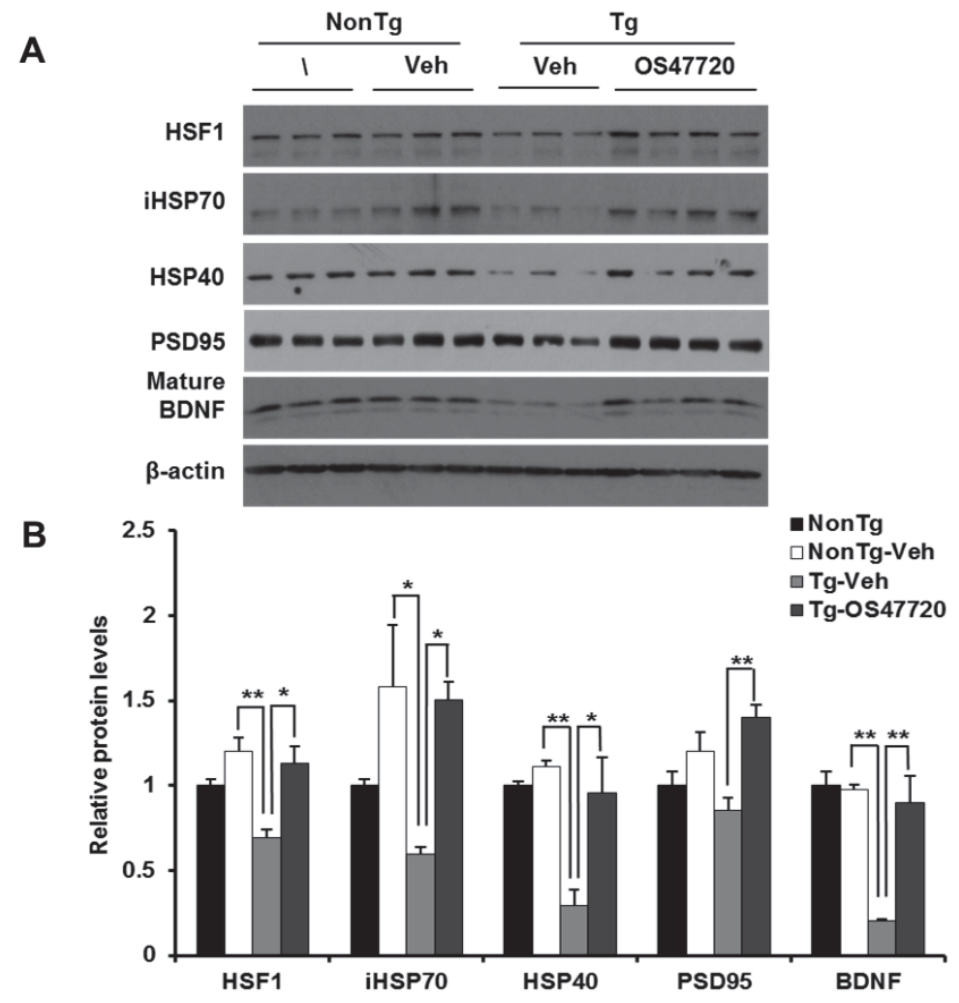

Figure 4-11 End Point Western Blot Analysis after 6-month Treatment of OS47720.

(A) Western blot analysis on post-mortem hippocampal tissue followed by quantification. 3 or 4 representative samples were shown. N=5-6. (B) Quantification analysis based on the Western blot images. One-way ANOVA was performed followed by Tukey's Post hoc test, NonTg-Veh vs NonTg-OS47720, Tg-Veh vs NonTg-Veh, Tg-Veh vs TgOS47720, $* \mathrm{P}<0.05, * * \mathrm{P}<0.01$ and $* * * \mathrm{P}<0.001$. Data are represented as means \pm SEM. 
A

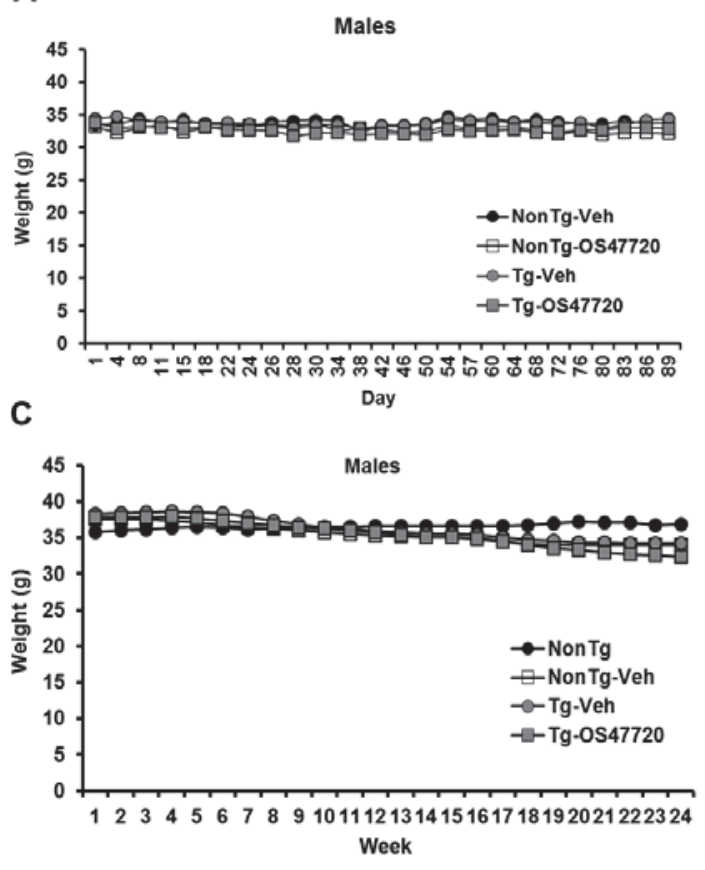

B

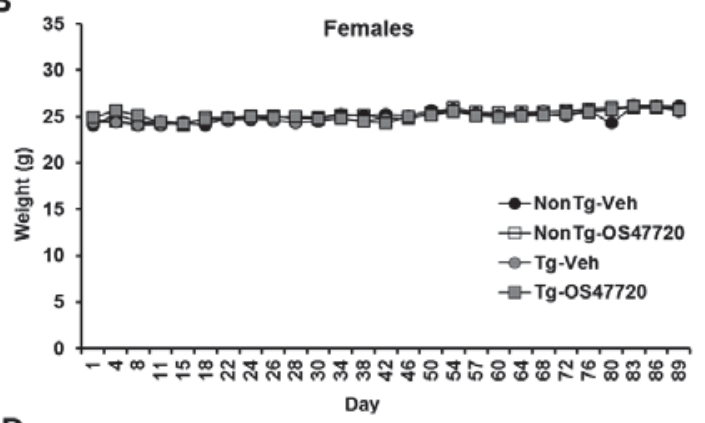

D

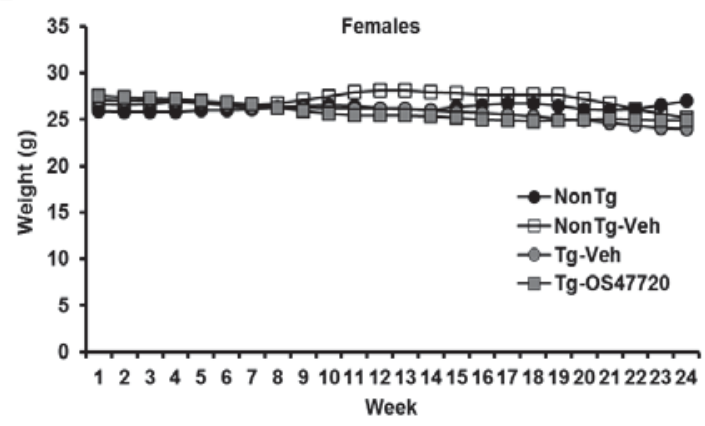

Figure 4-12 Body Weights from Two Cohorts of Tg2576 Mice with Chronic Treatment of OS47720.

(A, B) Weight data during 3 months of treatment ( $\mathrm{n}=20$ or 21). (C, D) Weight data during 6 months of treatment $(n=11-13)$. Males and females are presented separately. Weight was measured at indicated days or weeks shown on X-axis. 


\subsection{Summary}

In this chapter, a novel CNS-permeable Hsp90 inhibitor OS47720 was studied for its in vitro and in vivo efficacy. OS47720 produced an effect comparable to that of 17 AAG, as shown in the previous chapter, of inducing HSF1 nuclear translocation, heat shock-like responses and synaptic proteins. Similarly, structural changes in dendritic spines were also observed by IF staining.

In histologic analysis, OS47720 firstly exhibited a superb safety profile without any visible pathological changes in liver and kidney after in vivo use in mouse. LC-MS showed rapid brain accumulation of OS47720 within $1 \mathrm{~h}$ and confirmed its CNSpermeability after a single i.p. injection of OS47720. Although the compound gradually cleared from the brain after $24 \mathrm{~h}$, the pharmacodynamic effect of inducing iHsp70 and PSD95 lasted for at least 3 days, which was further confirmed in iHsp70-mPlum reporter mice. Most interestingly, hippocampal CA1 region exhibited the greatest response, compared with other regions within the hippocampus and cerebral cortex, which suggested a possible memory enhancement since the hippocampus is closely related to memory.

With behavior approaches, OS47720 firstly displayed protection on memory impairment induced by $\mathrm{A} \beta$ during contextual fear conditioning test. Chronic treatment of OS47720 for 3 months and 6 months of Tg2576 mice at early and middle symptomatic stages revealed completely rescued both a short-term memory (working memory) deficit in the cross maze and a long-term memory (spatial and learning memory) deficit in the Morris water maze. Post-mortem biochemical study revealed underlying molecular changes. All heat shock proteins, synaptic proteins, as well as BDNF were restored to the level comparable to non-Tg mice after OS47720 treatment. Additionally, OS47720 did not affect locomotor activity or anxiety-related behavior during chronic treatment. No significant body weight reduction was observed in all groups tested, indicating a feasible treatment range.

In conclusion, the CNS-permeable OS47720 compound provided convincing in vivo efficacy results without any systemic toxicity during various behavior tests. These findings verify and support the potential of a therapeutic strategy of targeting Hsp90 inhibition in AD. 


\section{CHAPTER 5. MECHANISTIC STUDY}

\subsection{Introduction}

The initial finding of application of Hsp90 inhibitors on cancer treatment relied on degradation of Hsp90 client proteins. Similar to GA, radicicol and its derivatives, OS47720 binds to the ATPase binding site located at the N-terminus of Hsp90 protein complex, leading to dissociation of Hsp90 and its client proteins. Released client proteins can be degraded by ubiquitin-proteasome system (UPS). Since many client proteins are cancer-related, these compounds were actively tested for their antitumor effect.

Many neurodegenerative diseases are associated with misfolded proteins and protein aggregates, which are the targets of some of the traditional drug development. Clearance of these disease causing misfolded protein aggregates is believed to be beneficial during neurodegeneration. Lots of evidence showed that Hsp90 inhibition was able to reduce the misfolded protein load. Luo et al. (2007) showed the reduction of phosphorylated tau in JNPL3 tau Tg mice by acute (single injection; $1 \mathrm{~d}$ ) treatment of the Hsp90 inhibitor PU-DZ8. In the same year, subchronic treatment (7 d) of the Hsp90 inhibitor EC102 in Htau mice reduced tau aggregates (Dickey et al., 2007). More recently, Labbadia et al. (2011) found that orally administrated Hsp90 inhibitor NVP-Hsp990 (Novartis compound) transiently reduced Huntingtin protein load. Although until there had been no in vivo work done to investigate of the effect of the Hsp90 inhibitor on A $\beta$ clearance, all previous work of Hsp90 inhibitor on other disease proteins implies a potential function of OS47720 on Tg2576. In the current chapter, we examined A $\beta$ level among experimental groups.

It is also known that heat shock proteins are protective to cells under internal or external stress (Brown, 2007). Direct overexpression of Hsp70 reduced polyglutamine aggregation in vitro transfected cells. In Drosophila, direct expression of Hsp70 suppresses polyglutamine-induced neurodegeneration in vivo (Warrick et al., 1999). Later study in mammalian cell models of HD, bacterial chaperone and yeast Hsp40 were found to reduce polyglutamine aggregation and prevent cell death (Carmichael et al., 2000). Upregulation of heat shock proteins by the pharmacological agent BRX-220 increased the survival of injured motor neurons and promoted morphological and functional improvement in the sensory system after peripheral nerve injury (Kalmar et al., 2002; Kalmar et al., 2003). Induction of heat shock proteins also delayed disease progression in ALS mice expressing a SOD1 mutant (Kieran et al., 2004). There were also findings suggesting Hsp90 inhibitors work as inducers of heat shock proteins to treat neurodegeneration. In a cell culture model of HD, treatment of GA induced expression of heat shock proteins (Hsp90, Hsp70 and Hsp40), and inhibited HD exon I protein aggregation in a dose-dependent manner. The effect was replicated by overexpression of Hsp70 and Hsp40 in a separate cell culture model of HD (Sitter et al., 2001).

HSF1 is known as a master transcriptional heat shock response regulator to induce heat shock proteins. OS47720 activated HSF1 nuclear translocation and transcriptional 
activity, which led to further investigation of HSF1-mediated regulation underlying OS47720's therapeutic effect in Tg2576 mice. Besides, inspired by 17-AAG study, novel regulation of HSF1 in synaptic proteins and BDNF was also examined in current experimental settings.

\subsection{Results}

\subsubsection{OS47720 Did Not Affect A $\beta$ Level and Neuroinflammation}

As shown by the in vivo efficacy study described above, OS47720 rescued memory loss in Tg2576 mice. The question remained as to how OS47720 improved memory. As an APP overexpressing mouse model of AD, Tg2576 mice had been well characterized by memory impairments closely correlated with the increase of $A \beta$ load in the cortex and hippocampus.

Immunohistologic staining was conducted using $A \beta$ antibody $4 \mathrm{G} 8$ in the second cohort of mice (6 months treatment). Representative images from staining revealed $A \beta$ plaque formation in the Tg-Veh group of mice, while treatment of 0S47720 did not significantly affect the $A \beta$ load (Figure 5-1A).

This unchanged A $\beta$ load was further confirmed by ELISA. Hippocampal tissues of mice from Tg-Veh group and Tg-OS47720 group were collected and processed according to described procedures. Soluble A $\beta$ 1-42, insoluble A $\beta$ 1-42, soluble A $\beta$ 1-40 and insoluble $A \beta 1-40$ were tested separately. There was no significant change in either soluble and insoluble $A \beta$ 1-42, or soluble and insoluble $A \beta 1-40$ after 6 month chronic OS47720 treatment (Figure 5-1B). Both immunohistological staining and ELISA demonstrated that OS47720 did not affect A $\beta$ production or secretion.

Neuroinflammation accompanies the pathological process of $\mathrm{AD}$ and plays an important role. However it is still not clear whether it is a causal or responsive event to disease pathology (Heneka et al., 2015). Typical neuroinflammation in AD involves an astrocytic response and microglial activation, which can be visualized by immunofluorescence staining of their respective protein markers glial fibrillary acidic protein (GFAP) and ionized calcium-binding adapter molecule 1 (Iba1). Representative images shown in Figure 5-1C revealed treatment of OS47720 did not change general neuroinflammation levels in the hippocampus and cerebral cortex.

\subsubsection{KRIBB11 Abolished Heat Shock-like Response and Contextual Conditioned Freezing Memory}

It was hypothesized that OS47720 improved memory through activation of HSF1. To test this hypothesis, HSF1 manipulation is desired. First, an HSF1-specific pharmacological inhibitor KRIBB11 was used to downregulate HSF1 protein expression 


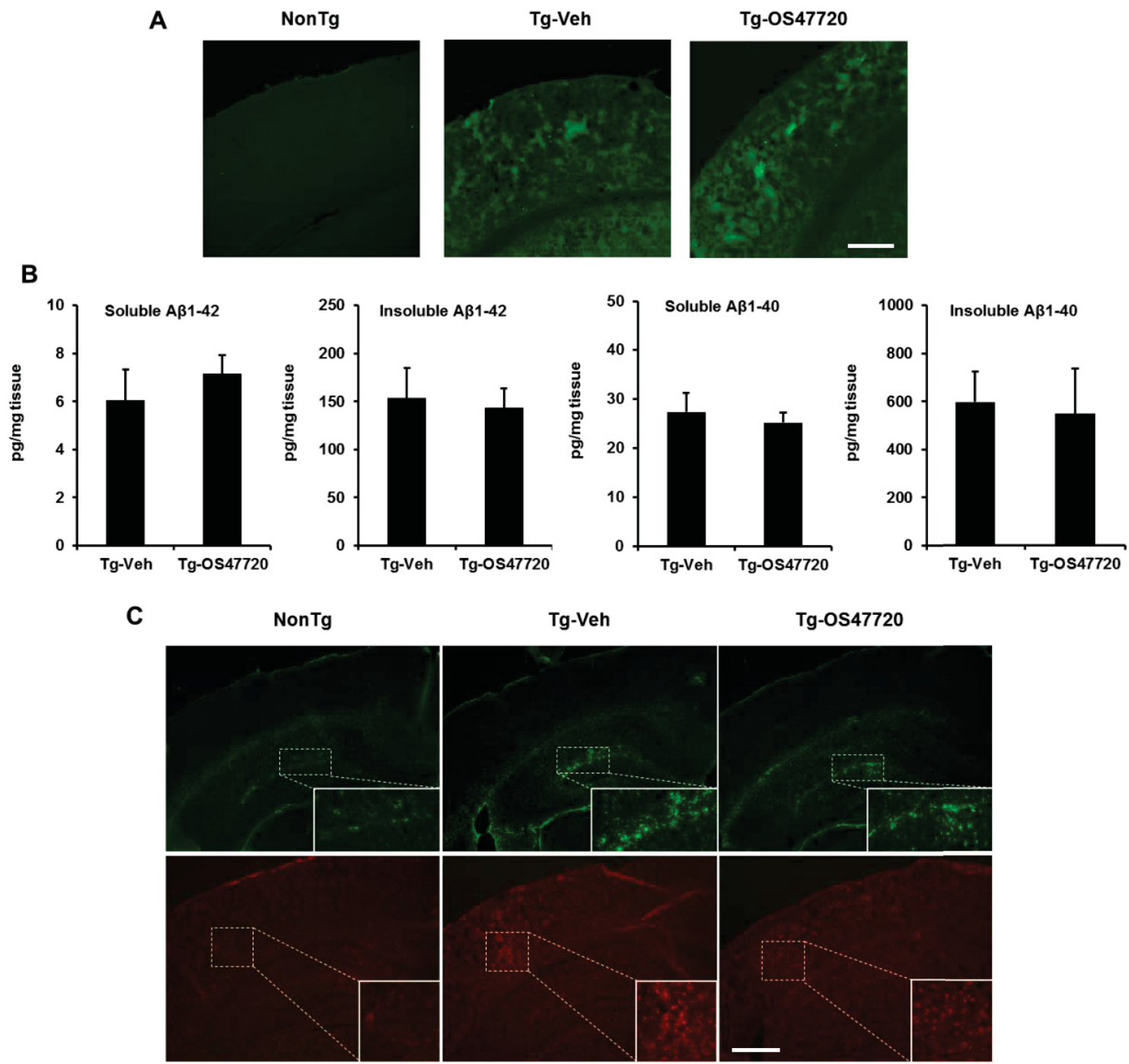

Figure 5-1 OS47720 Treatment Has No Effect on A $\beta$ and Plaque Load in 18 Month-old Tg2576 Mice.

(A) Representative fluorescence images from immuno-staining of $A \beta$ with $4 \mathrm{G} 8$ antibody in Tg2576 mouse brain (coronal). Scale bar is $200 \mu \mathrm{m}$. (B) ELISA of soluble and insoluble $A \beta 1-40$ and $A \beta 1-42$ using hippocampal tissue from Tg-Veh and Tg-OS47720 groups. (C) Representative fluorescence images from immuno-staining of GFAP (green; upper row) and Iba1 (red; bottom row) in Tg2576 mouse brain (coronal). Scale bar is 200 $\mu \mathrm{m}$. 
(Yoon et al., 2011). KRIBB11 was microinjected into mice at the same triple time points as indicated in Figure 3-4A during contextual fear conditioning training. $A \beta$ was also microinjected for side by side comparisons with KRIBB11.

During the training session, both $\mathrm{A} \beta$ and KRIBB11 treatment did not affect the fear acquisition (Figure 5-2A). During contextual fear retention, $A \beta$ treatment consistently reduced the percentage of freezing time at the $48 \mathrm{~h}$ and $72 \mathrm{~h}$ time points. Triple injections of KRIBB11 impaired contextual conditioned freezing memory even more severely than did $A \beta$ administration at both $48 \mathrm{~h}$ and $72 \mathrm{~h}$ time points, as evidenced by even lower percentage of freezing times recorded during contextual fear retention (Figure 5-2B). This was accompanied by marked reductions of HSF1, HSPs, and various synaptic proteins (Figure 5-2C and D). This finding suggests the requirement of HSF1 for the contextual conditioned freezing memory.

In order to further confirm the pharmacological effect of KRIBB11 in the brain, KRIBB11 was microinjected into the lateral ventricle through i.c.v. administration. Hippocampi tissues were collected and processed for Western blot analysis. Figure 5-2C and $\mathbf{D}$ showed that not only were heat shock proteins reduced, but also select synaptic proteins, including PSD95 and synapsin I, were greatly downregulated by KRIBB11.

\subsubsection{Upregulation and Downregulation of HSF1 by Viruses}

In order to address the role of HSF1 underlying OS47720's effect, adenoassociated viruses (AAV) were utilized. HSF1 was upregulated by using AAVH-BH which overexpressed a constitutively active HSF1 (AAVH-BH) (Jung et al., 2008), while HSF1 was downregulated by using AAVshRNAhsf1.

Both viruses were introduced into cultured primary neurons from hippocampus, with or without a co-treatment of OS47720 (100nM). Consistent with previous results, OS47720 treatment alone induced heat shock proteins, PSD95 and BDNF. AAVH-BH greatly increased protein levels of HSF1, which transcriptionally upregulated the expression of its downstream heat shock proteins expression (iHsp70, Hsp40 and Hsp27). Not surprisingly, PSD95 and BDNF were also upregulated. However co-treatment of OS47720 did not further increase heat shock proteins, PSD95 and BDNF. AAVshRNAhsf1 significantly reduced the expression of HSF1, which also transcriptionally reduced downstream heat shock protein expression. AAVshRNAhsf1 partially abolished OS47720's effect on increasing heat shock proteins, PSD95 and BDNF (Figure 5-3A). Quantification analysis is shown in Figure 5-3B. Therefore, upregulation or downregulation of HSF1 by AAVs resulted in opposite consequences of heat shock-like response (e.g., iHsp70), synaptic proteins (PSD95) and BDNF. 

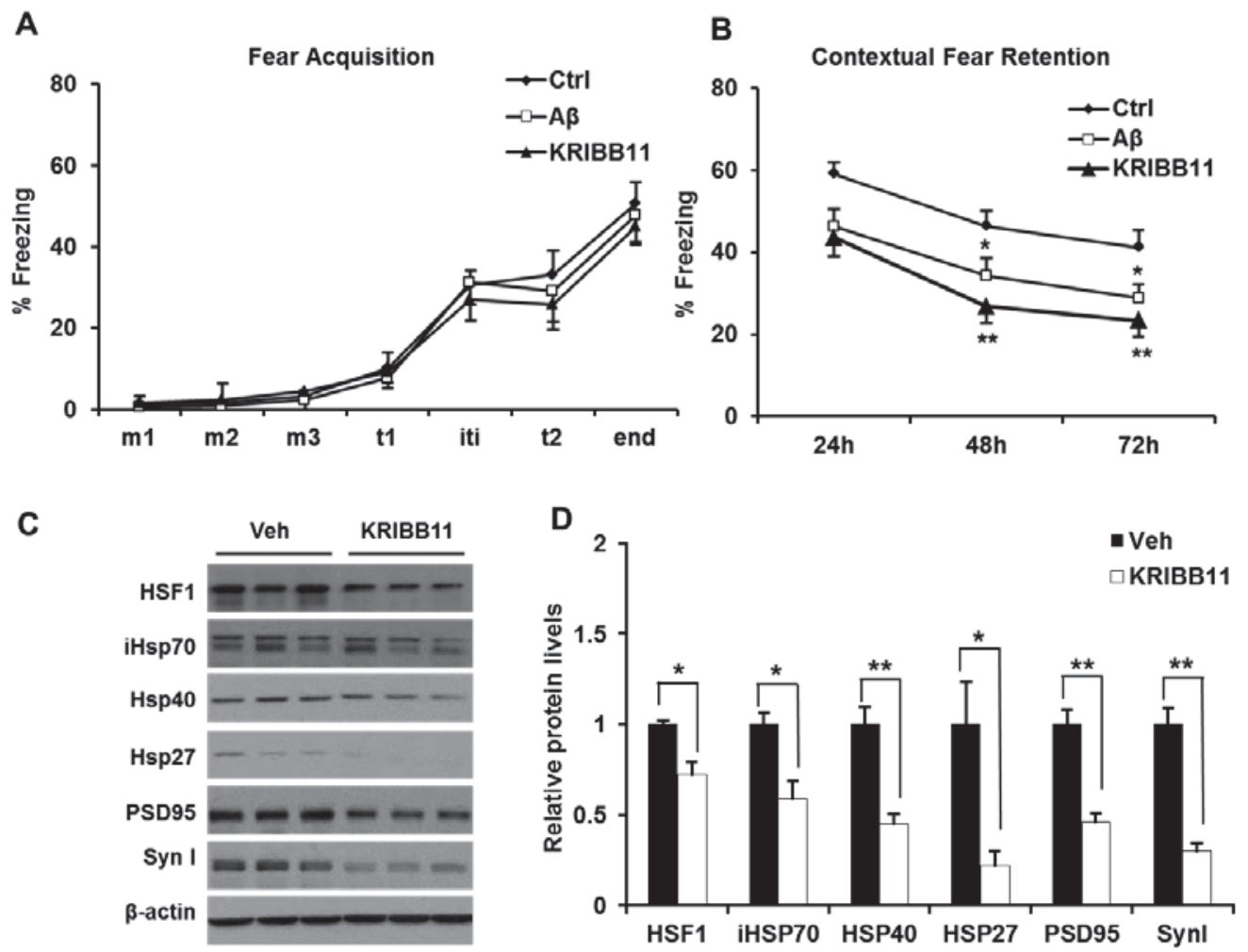

Figure 5-2 KRIBB11 Abolishes Contextual Fear Memory.

(A) Freezing time (\%) during fear acquisition (training) in the contextual fear conditioning test. Ctrl, control. (B) Recorded contextual fear retention (context retrieval) 24,48 and $72 \mathrm{~h}$ after training. One-way ANOVA was performed followed by Tukey's Post hoc test, ctrl vs $\mathrm{A} \beta,{ }^{*} \mathrm{P}<0.05$; $\operatorname{ctrl}$ vs KRIBB11, ${ }^{*} \mathrm{P}<0.01$. $\mathrm{n}=10-13$. (C) Western blot analysis on the hippocampi of mice treated with the pharmacological inhibitor KRIBB11, 4 h after i.c.v. injection. Protein levels of HSF1, iHsp70, Hsp40, Hsp27, synapsin I and PSD95 were determined. $\mathrm{n}=3$. (D) Histogram shows the statistical analysis (Two-sample student t-test, ${ }^{*} \mathrm{P}<0.05$ and $* * \mathrm{P}<0.01$ ). Data are represented as means \pm SEM. 

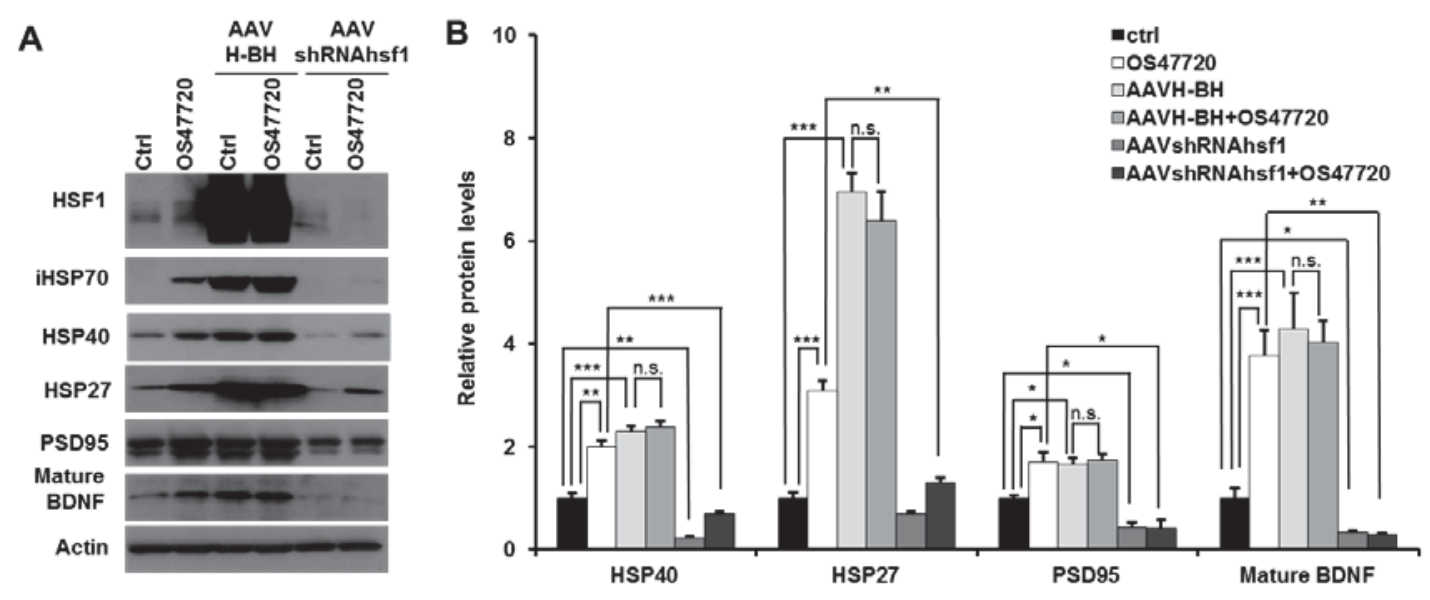

Figure 5-3 Upregulation and Downregulation of HSF1 by AAVH-BH and AAVshRNAhsf1 in Cultured Primary Neurons.

(A) AAVH-BH and AAVshRNAhsfl were introduced to cultured primary hippocampal neurons at 7-10 DIV. OS47720 (100 nM) was added 5-7 days after viral infection and was incubated for 24 hours. Western blot analysis was performed afterwards. (B) Statistical analysis of quantification using one-way ANOVA on 3 independent experiments, followed by Tukey's post hoc test, all groups were compared with control except the AAVshRNAhsf1+OS47720 group, which was compared with OS47720. n.s., non-significant. $\mathrm{n}=3$. Means $\pm \mathrm{SEM} . * \mathrm{P}<0.05, * * \mathrm{P}<0.01$ and $* * * \mathrm{P}<0.001$. 


\subsubsection{Downregulation of HSF1 by AAVshRNAhsf1 Abolished the Effect of OS47720}

Since both AAVs had been tested for their effect on regulation of HSF1 in in vitro cultured primary neurons, they were further introduced to in vivo study, where they were microinjected into mouse brain. It was hypothesized that OS47720's effect was mediated by HSF1. AAVshRNAhsf1 was first used to test if OS47720 could work independently of HSF1. AAVshRNAhsfl also contained a construct expressing EGFP that can be visualized under the fluorescence microscope.

Small volumes of AAVshRNAhsf1 $(1.5 \mu l)$ were microinjected bilaterally into brain hippocampi over 10 minutes using stereotaxic technique. Three weeks after injection, EGFP was observed specifically in the hippocampal region (Figure 5-4A). During contextual fear conditioning, the AAV-mediated downregulation of HSF1, diminished contextual conditioned freezing memory at both 48 and $72 \mathrm{~h}$ after training which could not be rescued by OS47720 (Figure 5-4B and C). In contrast, OS47720treated mice had slightly higher basal contextual conditioned freezing memory at these time points. An end-point study of the altered proteins revealed abolished Hsp40, PSD95, and mature BDNF by AAVshRNAhsf1 administration; changes that were not restored by OS47720 treatment (Figure 5-4D and E).

\subsubsection{HSF1 Heterozygous Mice Exhibited Impaired Memory}

HSF1 was further examined for its role in memory regulation by using HSF1 heterozygous mice. A breeding pair of heterozygous HSF1 mice was purchased from the Jackson laboratory. About 60 offspring were born from 13 litters. However the genotyping revealed that no knockout mice survived, mainly because of the critical role of HSF1 in development. Therefore adult (4-6 months old) HSF1 heterozygous mice (+/-) were used and compared in memory tasks with their WT littermate controls. Working memory was evaluated by cross maze. Recognition memory was evaluated by a novel object recognition task. Locomotor activity was measured by the open field test. Anxietyrelated behavior was measured by elevated plus maze.

During cross maze test, heterozygous mice $(\mathrm{N}=14)$ showed comparable total entries with WT control mice $(\mathrm{N}=12)$ (Figure 5-5A), but a significantly lower alternation rate, indicating impaired working memory (Figure 5-5B). The open field test revealed less locomotor activity in heterozygous mice as evidenced by the shorter distance travelled (Figure 5-5C). During the novel object recognition task, both groups of mice showed similar exploration times (Figure 5-5D). WT mice showed slightly higher exploration time of the novel object over an old object, while heterozygous mice did not differ between two objects (Figure 5-5E). WT mice showed a significantly higher discrimination index than heterozygous mice which indicates a better recognition memory (Figure 5-5F). Anxiety was not affected by lowered HSF1 in heterozygous mice as there was no difference among all parameters examined in elevated plus maze (Figure 5-5G and $\mathbf{H}$ ). Western blot analysis confirmed HSF1 protein reduction in 

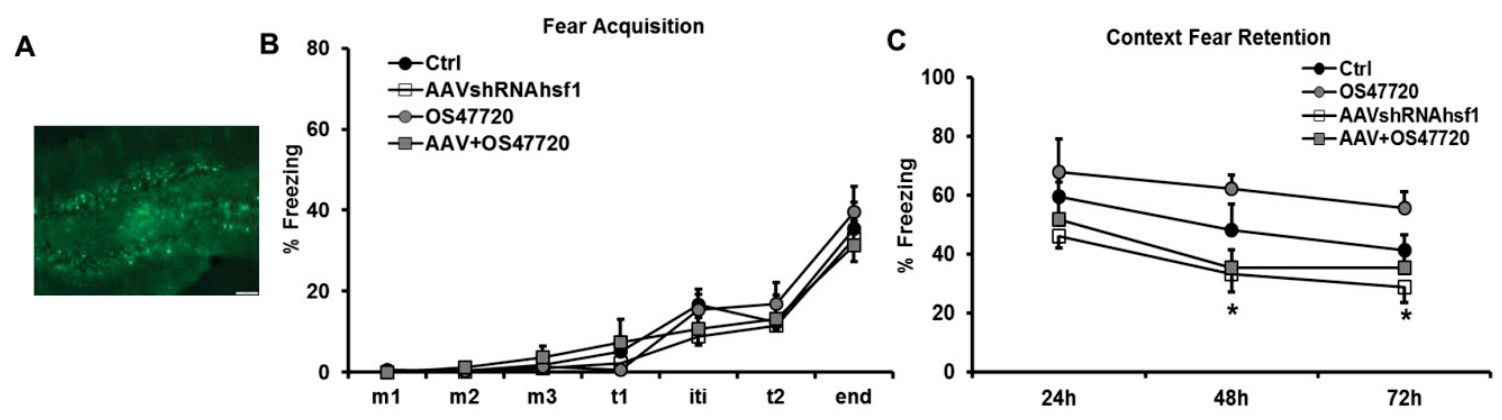

D

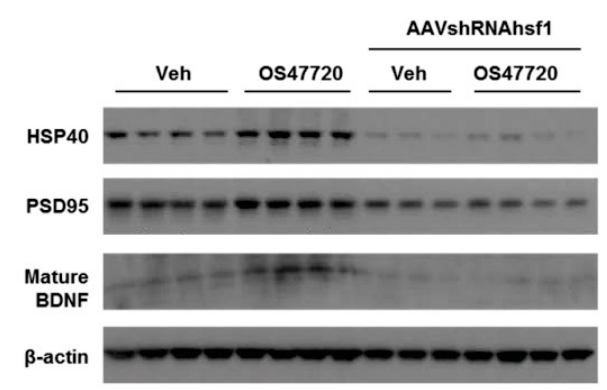

E

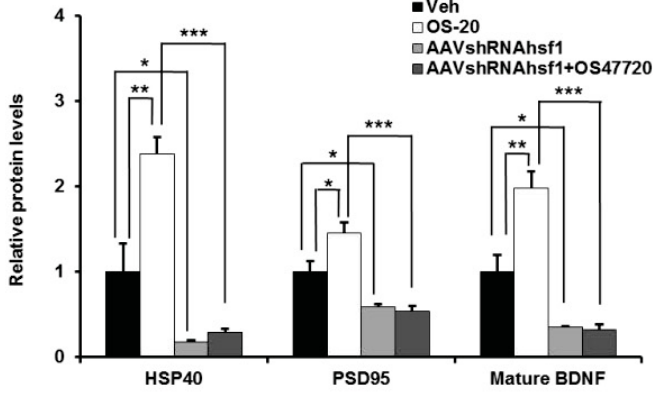

Figure 5-4 AAVshRNAhsf1 Abolishes the Effect of OS47720 in Contextual Fear Conditioning.

(A) EGFP expression in mouse brain 14 days after AAVshRNAhsf1 was stereotaxically microinjected into hippocampus. Scale bar is $100 \mu \mathrm{m}$. (B and C) Freezing time (\%) during fear acquisition and contextual fear retention in the contextual fear conditioning test. N=10-12. One-way ANOVA with Tukey's post hoc test, ctrl vs AAVshRNAhsf1. $* \mathrm{P}<0.05$. (D) Representative images of Western blot analysis after contextual fear conditioning. (E) Statistical analysis of the Western blot data. $n=6$. One-way ANOVA with Tukey's post hoc test, ${ }^{*} \mathrm{P}<0.05,{ }^{*} \mathrm{P}<0.01$ and ${ }^{* * *} \mathrm{P}<0.001$. Data are represented as means \pm SEM. 
A

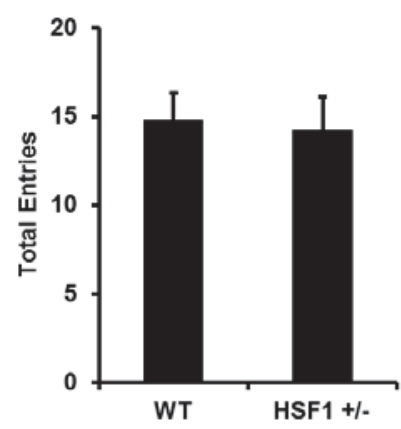

D

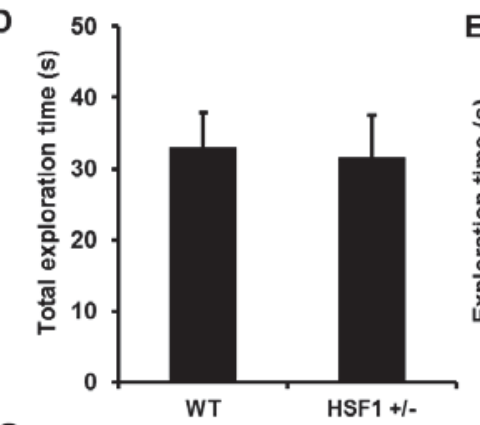

G

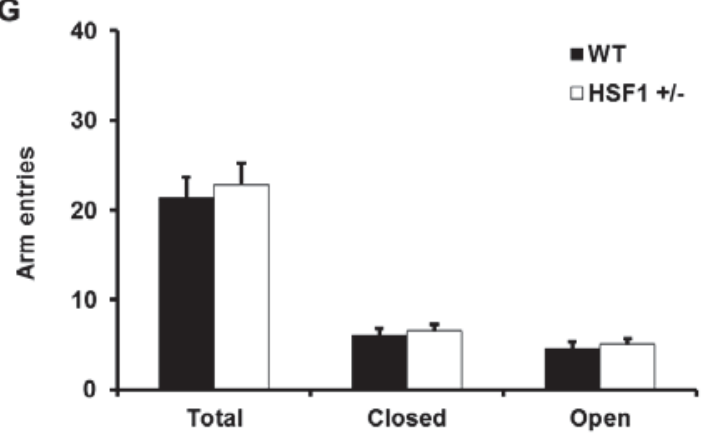

B
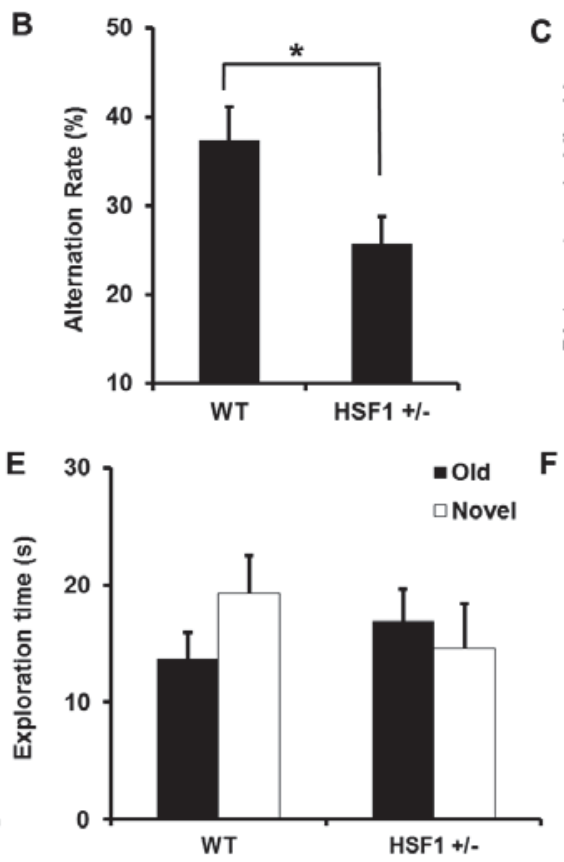

$\mathbf{H}$
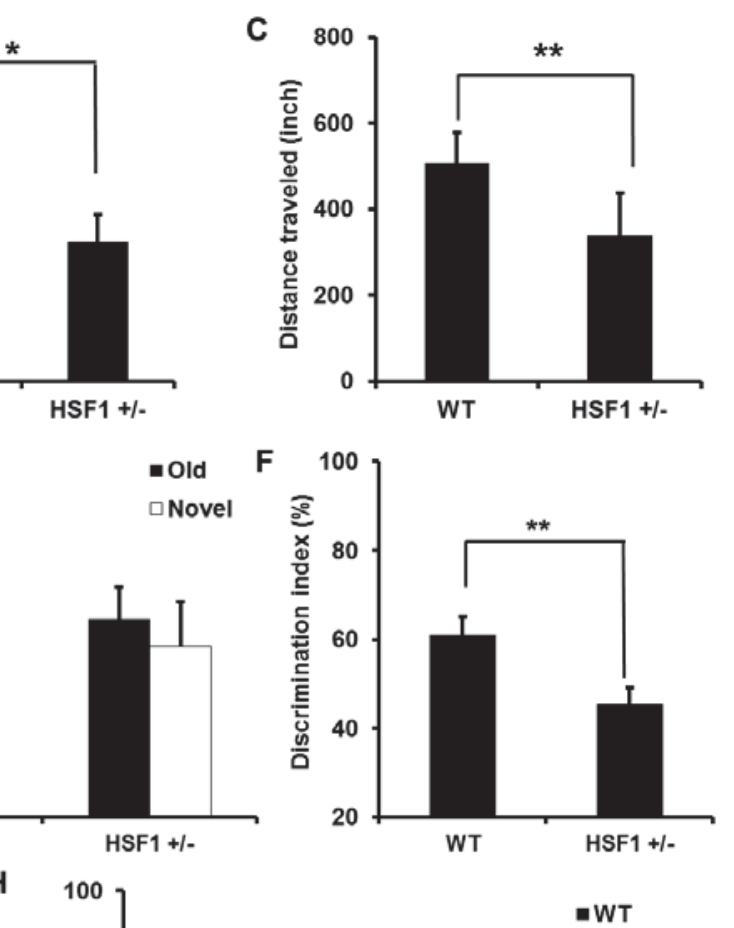

WT

口HSF1 +1-

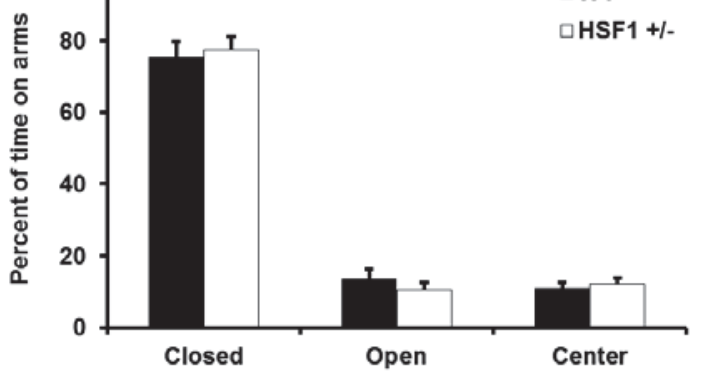

I WT +l- WT +l- WT +l-

HSF1

$\beta$-actin

Figure 5-5 Behavioral Tests on Young Adult HSF1+/- and Littermate Control Mice.

(A, B) Total entries and alternation rate (\%) in cross maze test. (C) Total distance (inch) traveled from open field test indicating locomotor activity. (D-F) Total object exploration time, exploration time and discrimination index in the novel object recognition task. $(\mathrm{G}$ and $\mathrm{H}$ ) Anxiety level was examined by arm (closed, open and total) entries and percentage of time on arms and center in the elevated plus maze. $\mathrm{N}=12$ or 14 , Means \pm SEM. Two-sample Student's t-test, $* \mathrm{P}<0.05$ and $* * \mathrm{P}<0.01$. (I) Images from Western blot analysis shown total HSF1 protein reduction in HSF1 +/- mice, compared with wildtype littermates' control. 
heterozygotes (Figure 5-5I). These results suggested HSF1 is required for memory process.

\subsubsection{Overexpression of HSF1 by AAVH-BH Slightly Improved Contextual Conditioned Freezing Memory}

Since it had been addressed that HSF1 is required for OS47720's effect by reducing HSF1 in previous experiments, the next question was whether overexpression of HSF1 has a comparable effect to OS47720. Mice were divided into the following four groups: 1. AAVYFP; 2. AAVH-BH; 3. AAVYFP+A $\beta$; 4. AAVH-BH+A $\beta$. AAVH-BH, control virus AAVYFP, and/or $A \beta$ were microinjected into hippocampus using stereotaxic technique. Each after 3 weeks, contextual fear conditioning was conducted. Figure 5-6A showed HSF1 was greatly upregulated, together with increases of iHsp70 and Hsp27. Interestingly, PSD95 and BDNF were also upregulated (Figure 5-6A). During contextual fear conditioning training, there was no difference in freezing time among groups (Figure 5-6B). During contextual fear retention, $A \beta$ again reduced contextual conditioned freezing memory. Overexpression of AAVH-BH slightly improved contextual conditioned freezing memory under both control and $A \beta$ conditions, though no significance was reached (Figure 5-6C). These results suggested that HSF1 contributed to the memory process.

\subsubsection{HSF1 Is Required for the Initial Stage of Memory Consolidation and Retrieval}

To determine whether the requirement of HSF1 is more crucial in the initial stage of memory acquisition and consolidation or for all stages (including retrieval and extinction), KRIBB11 was administered via implanted cannula at $1 \mathrm{~h}$ before and 1, 3, 6, 8, and $24 \mathrm{~h}$ after training. The most significant abrogating effect was observed at $1 \mathrm{~h}$ posttraining, and to a lesser degree at 3 and $6 \mathrm{~h}$ post-training. No effect was observed when injected at 8 or $24 \mathrm{~h}$ post-training (Figure 5-7). These results indicate that HSF1 is required for the initial stage of memory consolidation or retrieval.

\subsubsection{HSF1 Overexpression Induced BDNF Expression}

Because OS47720 was able to induce protein expression of BDNF, it would be interesting to see if BDNF could upregulated directly by overexpression of HSF1. Overexpression of a construct to constitutively activate HSF1 (Jung et al., 2008) in cultured hippocampal neurons markedly induced BDNF expression, as both pro and mature forms of BDNF protein levels were significantly increased (Figure 5-8A, B and C), so was BDNF mRNA level (Figure 5-8D). 


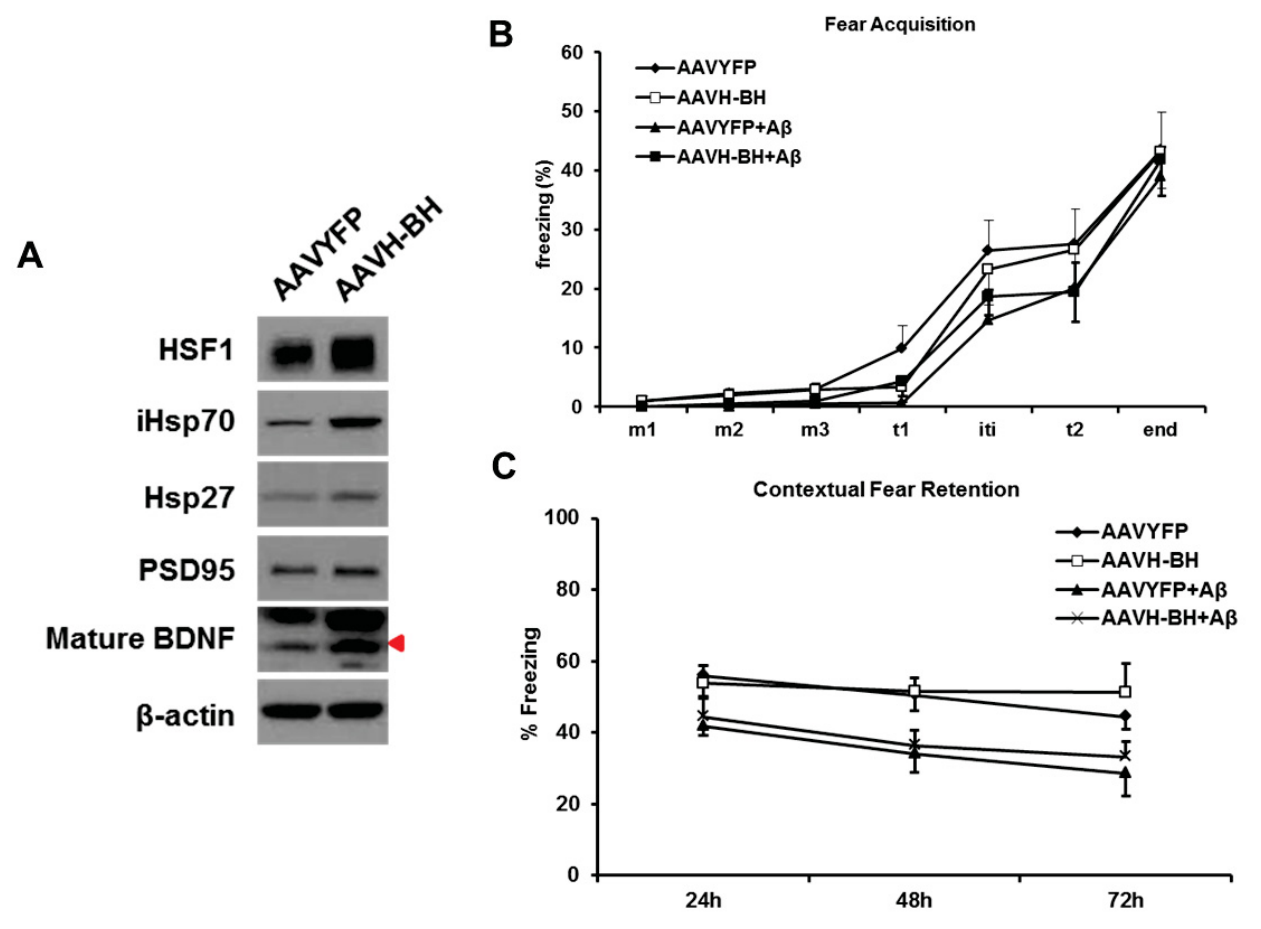

Figure 5-6 Overexpression of H-BH Did Not Exhibit Comparable Neuroprotection to OS47720 in Mice.

(A) Western Blot of hippocampus collected from mice receiving AAV H-BH for 3 weeks at CA1. (B and C) Fear acquisition and freezing time (\%) of contextual fear retention. $n=$ $10=13$. One-way ANOVA with Tukey's post hoc test was performed. No significance was found. Data are represented as means \pm SEM. 

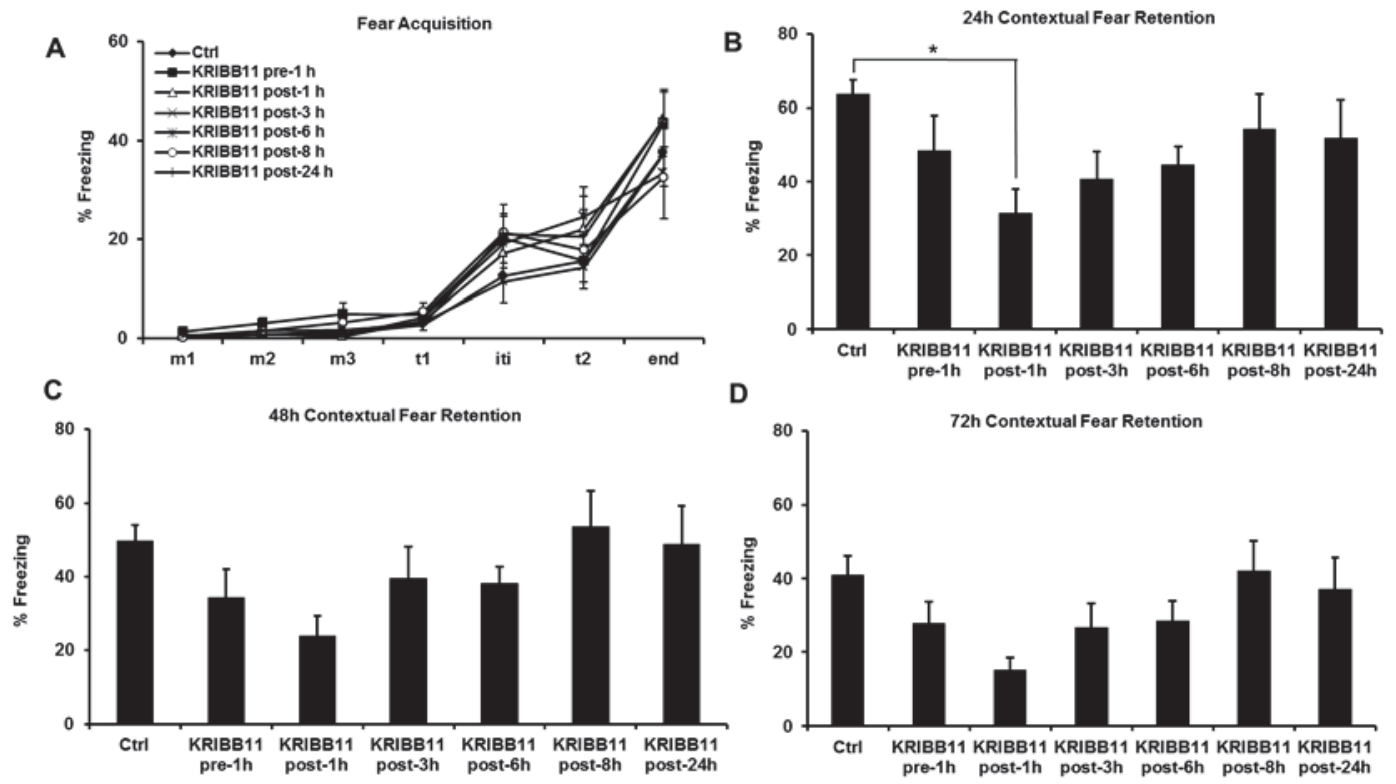

Figure 5-7 Time Course Study of KRIBB11 Treatment in Contextual Conditioned Freezing Memory Test.

KRIBB1 1 at the dose of $2 \mathrm{ul} / 2 \mathrm{mM}$ was injected through implanted cannulas to lateral ventricle (i.c.v.) for $10 \mathrm{~min}$. (A) Freezing time (\%) during fear acquisition (training). (BD) Freezing time (\%) of contextual fear retention, 24, 48 and $72 \mathrm{~h}$ after training. $\mathrm{n}=18$ (ctrl) or 6 (KRIBB11-treated groups). One-way ANOVA was performed followed by Tukey's post hoc test. $* \mathrm{P}<0.05$. Data are represented as means $\pm \mathrm{SEM}$. 

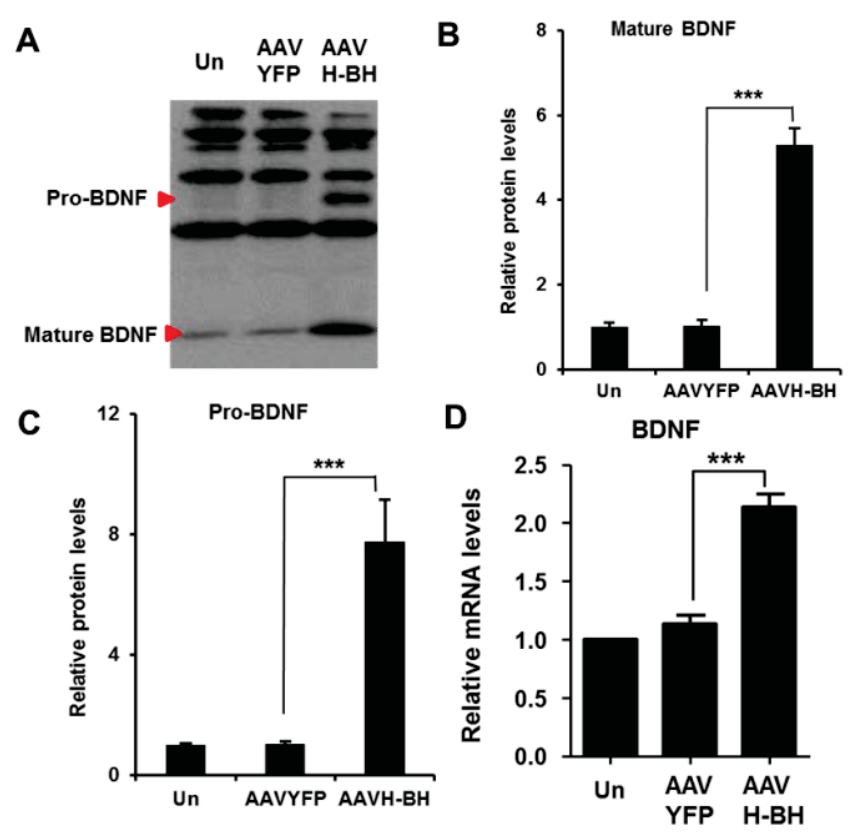

\section{Figure 5-8 BDNF Regulation by HSF1.}

Primary cultured hippocampal neurons were infected with AAVH-BH and AAV1-YFP control for 7 days. (A) Western blot analysis was performed to detect both precursor BDNF (pro-BDNF) and mature BDNF on 3 independent experiments. Un: untreated. Histograms show statistical analysis on changes of pro-BDNF (C) and mature BDNF (B). (D) The mRNA levels of HSF1 were determined by qRT-PCR after viral infection $\mathrm{N}=3$. One-way ANOVA with Tukey's post hoc test, ${ }^{* * *} \mathrm{P}<0.001$. Data are represented as means \pm SEM. 


\subsubsection{OS47720's Effect Bypasses cAMP/PKA and PI3K/AKT Pathways but Requires CaMKII}

To determine whether OS47720 induced BDNF upregulation via the classic cAMP-PKA-CREB pathway, cAMP levels were measured in cultured hippocampal neurons. OS47720 did not alter intracellular cAMP production even when a priming concentration of forskolin was given (Figure 5-9A). The lack of a role of the cAMPPKA-CREB, MAPK, and PI3K pathways in the formation of dendritic spines was confirmed by pharmacological inhibitors (i.e., RpcAMP and H89, U0126 and LY294002; Figure 5-9B)., These results are consistent with our previous reports showing reduced AKT levels upon Hsp90 inhibition in neurons (Chen et al., 2014). A CaMKII inhibitor did reduce the effect of OS47720 on dendritic spines. Statistical analysis revealed significant loss of dendritic spines using KN93, a selective CaMKII inhibitor, with or without co-treatment of OS47720, while no effect was observed by using its inactive derivative, KN92 (Figure 5-9C).

\subsection{Summary}

This chapter investigated molecular mechanism underlying the therapeutic effect from OS47720 chronic treatment.

Most Hsp90 inhibitors display neuroprotection in neurodegenerative diseases by degrading misfolded proteins or aggregates. In contrast, OS47720 did not significantly affect $A \beta$ load and general neuroinflammation after examining hippocampal $A \beta$ level in Tg2576 mice. Downregulation of HSF1 by both a pharmacological inhibitor KRIBB11 and by AAVshRNAhsf1, abolished OS47720's effects in the memory tests. Meanwhile, HSF1 overexpression by AAVH-BH partially increased contextual conditioned freezing memory. Moreover, HSF1 heterozygous mice showed impaired memory suggesting the requirement of HSF1 in memory regulation. Time course study of KRIBB11 treatment revealed that HSF1 is required for the initial stage of memory consolidation or retrieval. Further biochemical study showed BDNF was directly regulated by HSF1 at the transcription level. Taken together these results indicate the existence of an HSF1mediated mechanism underlying OS47720's effect, which required CaMKII but bypassed the several important signaling pathways, including the cAMP/PKA and PI3K/AKT pathways. 

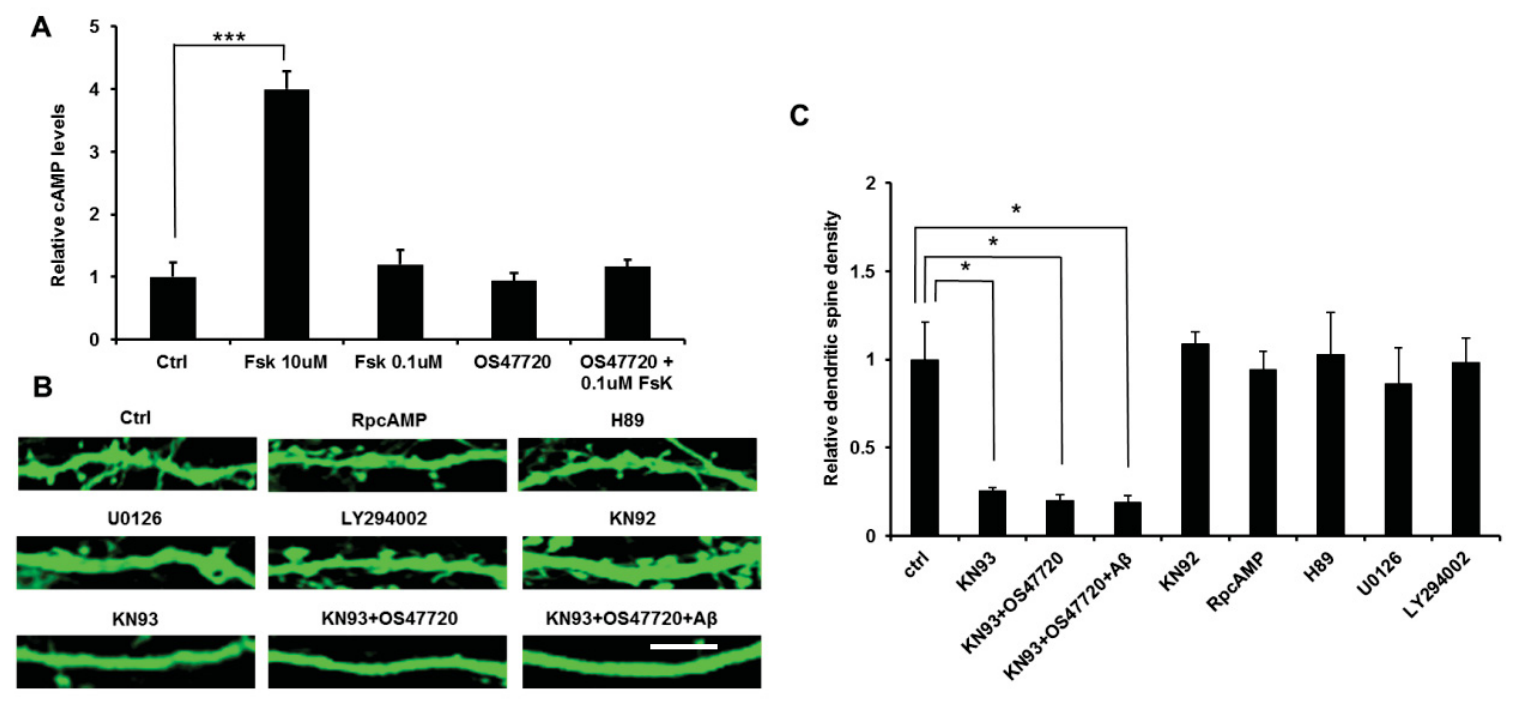

Figure 5-9 Hsp90 Inhibition Bypasses Several Major Signaling Pathways. (A) Primary hippocampal neurons (21 DIV) were used for studying the impact of OS47720 treatment on cAMP levels. Foskolin (Fsk) at $10 \mu \mathrm{M}$ and $0.1 \mu \mathrm{M}$ was used as positive control. cAMP levels were determined 15 min after Fsk and OS47720 treatment, using the HitHunter cAMP Assay for Small Molecules kit (DiscoveRx, Inc.) following the manufacturer's instructions. Data are presented as the result of 3 independent experiments (triplicated). (B) Confocal imaging of dendritic spines at $24 \mathrm{~h}$ after treatment of various pathway inhibitors in 14 DIV cultured primary hippocampal neurons. Inhibitors includes U0126, a selective inhibitor of the MAP kinase kinases/ MEK1 and MEK2; H89, PKA Kinase Inhibitor; Rp-cAMPS, a potent and specific competitive inhibitor of the activation of PKA by cAMP; LY294002, phosphatidylinositol 3-kinase (PI3K) inhibitor; KN93, a selective inhibitor of CaMKII; KN92, structural analog \& inactive form of KN-93. All inhibitor compounds were used at $10 \mu \mathrm{M}$. Scale bar is $5 \mu \mathrm{m}$. (C) Spine quantification based on 10 fields per slide. $n=3$. One-way ANOVA was performed followed by Tukey's post hoc test. $* \mathrm{P}<0.05$. 


\section{CHAPTER 6. DISCUSSION, CONCLUSIONS AND FUTURE DIRECTIONS}

\subsection{Discussion}

\subsubsection{Investigation of Hsp90 Inhibitors in Neurodegenerative Diseases}

Targeting Hsp90 with chemical inhibitors has long been an area of investigation in treating various types of cancer (Neckers, 2002; Whitesell and Lindquist, 2005). Many of these inhibitors are currently undergoing clinical trials, as in the case of SNX-5422 in (phase I), AUY-922 in (phase I/II), PU-H71 (phase I), etc. Limited clinical success has been achieved with several agents in certain types of tumors. Currently there is a lack of clinicaly approved Hsp90-targeting agents for cancer therapy.

Hsp90 inhibitors have also long been investigated for treatment of neurodegenerative diseases. While much of the experimental data has been obtained from in vitro studies, very limited success was achieved in vivo. Ansar et al. (2007) investigated a small molecule novobiocin analogue called A4, a C-terminus inhibitor of Hsp90, which induces Hsp90 protein overexpression, and also upregulates Hsp70 expression. A4 also showed neuroprotection against $\mathrm{A} \beta$ induced toxicity in cultured primary cortical neurons (Ansar et al., 2007). That group later confirmed that both A4 and another novobiocin analogue, KU32, exhibited neuroprotective properties against $\mathrm{A} \beta$-induced cell death as measured, by lactate dehydrogenase (LDH) activity assay in differentiated SH-SY5Y neuronal cells (Lu et al., 2009). Tau-pathology has also been tested after Hsp90 inhibition from two other groups. In 2007, Dickey et al. targeted Hsp90 inhibition by the inhibitor EC102, showing preferential degradation of phosphorylated tau protein in the following models: Chinese hamster ovary (CHO) cells and human embryonic kidney (HEK293) cells overexpressing P301L mutant tau, in Hela cells overexpressing V5-tagged tau (V5-tau), and in AD transgenic mice (Htau mice) expressing human tau protein. All of these aforementioned models produced the 6 splice variants of the human tau protein, representing progressive pathogenesis of tauopathies (Dickey et al., 2007). In the meantime, it was Luo et al. (2007) who first demonstrated a reduced level of phosphorylated tau in cultured embryonic primary cortical neurons and COS-7 cells transfected with human tau cDNAs with P301L mutation, by using their laboratory synthesized Hsp90 inhibitor PU24FCl. Besides the in vitro findings, they also found a reduction of aggregated and hyperphosphorylated tau in an AD mouse model representing tauopathy (JNPL3 mice) by using a blood-brain barrier (BBB) permeable Hsp90 inhibitor PU-DZ8 (Luo et al., 2007). Other diseases with mutated proteins have also been investigated. Riedel and his colleagues reported that 17-AAG significantly reduced the formation of $\alpha$-synuclein aggregates in an oligodendroglia cell line (OLN-93 cells) overexpressing human A53T mutated $\alpha$-synuclein (Riedel et al., 2010). It was around the same time that Putcha and her colleagues used an Hsp90 inhibitor (SNX-2112) to prevent $\alpha$-synuclein induced toxicity (Pucha et al., 2010). In vivo pharmacokinetic and pharmacodynamic assessments of this SNX compound were also tested by this group; however no further in vivo efficacy was reported thereafter (Putcha et al., 2010). 
Mutations in leucine-rich repeat kinase-2 (LRRK2) are well recognized as common causes of late-onset PD. 17-AAG had been discovered to be capable of reducing LRRK2 in SH-SY5Y cells (Ko et al., 2009). Using GA, 17-AAG, and radicicol in a primary culture model of familial amyotrophic lateral sclerosis (FALS), researchers found reduced aggregation of SOD1G93A, a distinguishing pathological and molecular feature of FALS (Batulan et al., 2006). Jinwal's group demonstrated a clearance cascade for the TDP-43 protein through an internal autophagy system after Hsp90 inhibition by 17-AAG in HeLa cells which had V5-tagged tau stably transfected (Jinwal et al., 2012). For studies in HD and other polyQ diseases, Sittler and his colleagues discovered that in an in vitro cell culture model, COS-1 cells overexpressing huntingtin exon 1 protein with 72 glutamines [HD72Q], GA exhibited inhibition of protein aggregation of huntingtin (Sittler et al., 2001). Gregory's group demonstrated that 17-AAG treatment reduces aggregation of ALS related protein TDP-43 in Drosophila (Gregory et al., 2012). Labbadia with his colleagues discovered a transient reduced Htt load in a Huntington's disease mouse model, R6/2 mice, after administration of NVP-Hsp990, which is a Novartis compound of Hsp90 inhibition potency (Labbadia et al., 2011). It has been reported that GA reduced aggregation and promoted degradation of the abnormally glutamine-expanded androgen receptor (AR) in mouse embryonic fibroblasts (MEFs) (Thomas et al., 2006). Furthermore, Waza et al. (2005) found that 17-AAG was an effective treatment for spinobulbar muscular atrophy (SBMA), importantly being able to avoid issues dealing with CNS permeability (Waza et al., 2005).

In addition to systemic toxicity, BBB penetrability has been a large obstacle in the development of drugs for CNS-related applications. Molecular size and lipophilicity are chief among physicochemical properties as the primary factors determining BBB penetrability. There is so far evidence of two CNS-permeable Hsp90 inhibitors that have successfully reduced tau aggregation with feasible toxicity profiles (Table 6-1). Luo et al. (2007) tested a single i.p. injection of PU-DZ8 compound on P301L tau transgenic (JNPL3) mice and found reduced tau aggregation after $24 \mathrm{~h}$ of treatment (Luo W et al., 2007). Dickey et al. (2007) observed reduced tau aggregation after 7 days of treatment of another Hsp90 inhibitor EC102 (Dickey et al., 2007). Both of these instances presented controversy in that they were either treated too acutely (single i.p. injection) or too briefly (only 7 days). Surprisingly, the Novartis compound NVP-Hsp990 (and NVP-AUY922 from the same series) was ineffective against Huntington's Htt R6/2, despite its efficacy in vitro (Labbadia et al., 2011). Interestingly, an earlier member of the same OS series of Hsp90 inhibitors (OS47701) also did not demonstrate efficacy in the ALS SODG93A4 model due to minimum iHsp70 induced in the spinal cord (Cha et al. 2014). The modest neuronal stress response after Hsp90 inhibition, as assessed by the induction of iHsp70, indicates infeasibility of using Hsp90 inhibitors in treating aggressive proteinopathies such as HD and ALS. The current study presents data demonstrating successful chronic (two cohorts of mice at 3 months and 6 months respectively) treatment of earlysymptomatic and middle-symptomatic staged Tg2576 mice with a CNS-permeable and non-toxic Hsp90 inhibiting compound. Based on our data, we conclude that Hsp90 inhibitors have unique therapeutic value for $\mathrm{AD}$ because of their unique protective mechanism on synapses in preserving both synaptic structures and functions. The potential therapeutic implications of these findings are boundless, and are only further 
Table 6-1 Summary of the In vivo Data Using Hsp90 Inhibitors in Neurodegeneration in Mouse Models.

\begin{tabular}{|c|c|c|c|c|c|c|}
\hline Compound/Source & $\begin{array}{c}\text { Client protein/ } \\
\text { Neurodegenerative } \\
\text { disease }\end{array}$ & $\begin{array}{l}\text { Administrative } \\
\text { regimen }\end{array}$ & $\begin{array}{c}\text { HSPs } \\
\text { induction } \\
(\text { e.g., } \\
\text { iHsp70) } \\
\end{array}$ & In vivo effect & $\begin{array}{l}\text { Improved } \\
\text { disease } \\
\text { syndrome }\end{array}$ & References \\
\hline $\begin{array}{l}\text { PU-DZ8/ Sloan- } \\
\text { Kettering }\end{array}$ & $\begin{array}{l}\text { Tau/AD } \\
\text { (TauP301L/JNPL3) }\end{array}$ & $\begin{array}{l}\text { i.p. injection; } 75 \mathrm{mg} / \mathrm{kg} \text {; } \\
\text { single injection; } \\
\text { duration: } 1 \text { day }\end{array}$ & n.d. & $\begin{array}{l}\text { Reduce tau- } \\
\text { P/aggregation }\end{array}$ & n.d. & $\begin{array}{l}\text { Luo et al. PNAS. } \\
(2007)\end{array}$ \\
\hline $\begin{array}{l}\text { EC102/ Conforma } \\
\text { Therapeutics. }\end{array}$ & $\begin{array}{l}\text { Tau/AD (humanized } \\
\text { Htau ) }\end{array}$ & $\begin{array}{l}\text { i.p. injection; } 200 \mathrm{mg} / \mathrm{kg} \\
\text { daily for } 7 \text { days }\end{array}$ & good & $\begin{array}{l}\text { Reduce tau- } \\
\text { P/aggregation }\end{array}$ & n.d. & $\begin{array}{l}\text { Dickey et al. J. } \\
\text { Clin. Invest. (2007) }\end{array}$ \\
\hline NVP-Hsp990/ Novartis & $\mathrm{Htt} / \mathrm{HD}(\mathrm{R} 6 / 2)$ & $\begin{array}{l}\text { oral gavage; } 12 \mathrm{mg} / \mathrm{kg} \\
\text { daily for } 9 \text { weeks }\end{array}$ & disrupted & $\begin{array}{l}\text { Transiently } \\
\text { reduce Htt load }\end{array}$ & No & $\begin{array}{l}\text { Labbadia et al. J. } \\
\text { Clin. Invest. (2011) }\end{array}$ \\
\hline $\begin{array}{l}\text { OS47720 (NXD30020)/ } \\
\text { OncoSynergy }\end{array}$ & $\mathrm{A} \beta / \mathrm{AD}(\mathrm{Tg} 2576)$ & $\begin{array}{l}\text { i.p. injection; } 100 \\
\mathrm{mg} / \mathrm{kg} \text {; two or three } \\
\text { times per week for } 3-6 \\
\text { months }\end{array}$ & modest & $\begin{array}{l}\text { Rescue } \\
\text { memory } \\
\text { (Behavior) }\end{array}$ & Yes & Herein \\
\hline
\end{tabular}

Notes: Detailed Summary of the in vivo data from the CNS-permeable Hsp90 inhibitors in various mouse models of neurodegenerative diseases. n.d., not determined. 
encouraged by the favorable pharmacokinetics and pharmacodynamics of this compound (OS47720) (Table 4-1).

\subsubsection{Heat Shock Response in Neuronal Cells}

In the past decade, the stress response to heat or Hsp90 inhibitors have generally been considered to be blunted in neurons. The inability to induce a heat shock response has been documented in mouse and rat primary culture neurons. In fact, as early as 1996, Marcuccilli et al. found that primary cultured rat hippocampal neurons were unable to produce a heat shock response when subjected to hypothermia. However, a robust heat shock response was found in secondary cultured rat glial cells (Marcuccilli et al., 1996). In 2003, Batulan et al. (2003) discovered that cultured motor neurons failed to induce Hsp70 after excitotoxic glutamate, so did spinal cords from an ALS transgenic mouse model (SOD1G93A) and sporadic or familial ALS patients. When a constitutively active form of HSF1 was overexpressed, enhanced induction of Hsp70 was observed (Batulan et al., 2003). These results demonstrated that a failure to activate HSF1 led to a blunted response or created a high threshold for the neuronal heat shock response. It has thus been well accepted that an impaired heat shock response exists in neurons.

While much of the data was generated through neuroblastoma or rodent primary neuronal culture models, admittedly there has been a lack of in vivo data. However, recently Carnemolla et al. (2015) performed an extensive and in-depth analysis of the heat shock response in the mouse brain and found that the brain was capable of maintaining a fairly strong heat shock response throughout its lifespan. This was either an indication that the heat shock response was not compromised in neuronal cells, or rather that non-neuronal cells were taking over the charge. The group further separated neurons and non-neuronal cells from mouse brains and performed extensive and in-depth analysis of heat shock response after Hsp90 inhibition by NVP-Hsp990. By using a neuronal purifying technique, they induced a heat shock response in striatal projection neurons. Their findings of an existing competent heat shock response in neurons ultimately supports our idea of developing therapeutics to neurodegenerative diseases targeting the heat shock pathways in neurodegenerative diseases.

Similarly, the ex vivo data (Figure 4-5) on the iHsp70 reporter mice after either single or continual OS47720 treatment also indicated a relatively weakened, but not blunted, neuronal HSF1/iHsp70 response in the brain relative to the liver and muscle. The hippocampal CA1 region demonstrated the greatest HSF1/iHsp70 response in the brain, while spinal cord motor neurons demonstrated almost undetectable responses (Figure 4-4). This finding is consistent with previous reports that motor neurons have a comparatively high threshold for activation of genes for heat shock proteins, which may explain why this specific neuronal type is the most vulnerable and undergoes the most rapid degeneration (Cha et al., 2014). There are several possibilities for the weak heat shock response in most neurons, which may not be mutually exclusive: 1) the presence of intrinsic repressor(s) at the HSE sites. HSF-4a and HSF-4b were identified to be repressors of HSF1-mediated transcription by binding with HSEs (Zhang et al., 2001). 
HSF-4a repressed the formation of HSF1 nuclear granules. 2) HSF1 itself is differently regulated in neurons. HSF1 is differently activated in development cells and in stressed cells. A unique trimerization mechanism underlies the effect of HSF1 protecting neurons from stress. It does not require the involvement of the expression of heat shock proteins such as Hsp70 or Hsp90 (Verma P et al., 2014). 3) The existence of an intrinsically inert neuronal iHsp70 promoter, which may interfere with the binding activity of HSF1. It was confirmed by Fernandez-Funez P et al. in a Drosophila model (Fernandez-Funez et al., 2014). These possibilities require further investigation.

In the current study, we did not detect any observable effects of reducing amyloid A $\beta$ load in Tg2576 mice after 3 or 6 months OS47720 administration, despite several reports of anti-A $\beta$ effects caused by other Hsp90 inhibitors (Lu et al., 2009; Ansar et al., 2007). These seemingly contradictory results may reflect intrinsic differences between in vitro and in vivo experimental systems, because the majority of reports indicating A $\beta$ reducing effects were derived from in vitro studies lacking the proper neuroglial microenvironment and the systemic metabolism of in vivo drug administration. We believe that the lack of A $\beta$ reduction after chronic OS47720 administration is due to the moderate induction of stress responsive proteins (iHsp70 and Hsp40) by this drug (Figure 4-2 and Figure 4-4). It has been acknowledged that high levels of iHsp70 (and perhaps other co-chaperones like Hsp40) facilitates disease protein (A $\beta, \mathrm{Htt}$, etc.) degradation (Magrané et al., 2004), for example, Hsp70 overexpression in AD mice crossed with an Hsp70 transgenic (Hoshino et al., 2011; Bobkova et al., 2014), Hsp70 overexpression in HD R6/2 mice crossed with Hsp70 overexpression mice (Hay et al., 2004), and DNAJ (Hsp40) overexpression on Htt degradation in R6/2 mice (Labbadia et al., 2012). However, it appears that such high expression levels of chaperone proteins in animal models are ultimately unachievable via an Hsp90 inhibition approach.

\subsubsection{Novel Findings: HSF1-mediated Mechanism on Synapses}

Based on previously published works, most of the investigations of Hsp90 inhibitors on AD were focused on the co-chaperone functions of Hsp90, in which Hsp90 inhibitors lead to disassociation of Hsp90 and its client proteins. Upon release from the Hsp90 complex, these client proteins could be degraded through the UPS. Previous studies in yeast, drosophila, and mammalian cells revealed that HSF1 is not only in charge of encoding genes of chaperone proteins under stressed conditions, such as heat shock, oxidative stress, heavy metal etc., but also its expression is involved in several important cellular events including signal transduction, protein degradation, ion transportation, energy production, carbonhydrate metabolism, vesicular transportation and cytoskeleton formation (Gonsalves et al., 2011; Hahn et al., 2004; Trinklein et al., 2004). HSF1 is the major isoform that has been linked to chaperone protein dependent mechanisms of treating neurodegenerative diseases.

Current studies have identified that HSF1 plays a pivotal role in not only regulation of the traditional heat shock response, but also regulation of synaptic activity

and functions. OS47720 testing will certainly help clarify the previously unexamined role 
of HSF-1 mediated synaptic regulation. From a biochemical perspective, HSF1 overexpression significantly enhanced the synaptic protein PSD95. It should be noted that BDNF was also greatly upregulated. Meanwhile, downregulation of HSF1 by either the pharmacological inhibitor KRIBB11 or by AAVshRNAhsf1 abolished OS47720's effect on synaptic proteins and BDNF. These results lead to the conclusion that HSF1 regulates synaptic activity and function, and that HSF1 is required for effective OS47720 activity on synapses.

Our work demonstrates for the first time that OS47720, as a novel CNSpermeable Hsp90 inhibitor, has the potential to be an effective therapeutic for the AD mouse model Tg-2576, by repairing synaptic structure, reestablishing neuronal contact and rescuing impaired memory, via an HSF1-mediated regulation.

\subsubsection{Transcriptional Regulation by HSF1: Potentially Another Master Transcriptional Factor for Memory}

Pharmaceutical companies are currently investing in research for "Memory pills", which are designed not only to rescue severe memory deficits caused by brain dementia like $\mathrm{AD}$, but also to be used as a regular treatment for memory ailments in elders with mild memory loss, or for those who just want to prevent memory decline. For this purpose, Drs. Gary Lynch and Gary Rogers came up with the idea of boosting neurotransmitter (glutamate) signaling. Repeated glutamate exposure activates AMPA receptors, and triggers NMDA receptor activation, allowing a calcium influx. Receptor activation induces synaptic change, which is what is believed to be the basis of memory encoding and consolidation (Lynch, 2002; Lynch et al., 2011). The influential idea that cAMP and CREB regulated memory formation was formulated by the 2000 Nobel Prize winner Dr. Eric Kandel and his rival scientist Dr. Tim Tully. cAMP can induce a large pool of proteins that strengthen neuronal connections. Most importantly, cAMP activates CREB which triggers transcription of several genes involved in memory processes (Barco et al., 2003; Jackson et al., 2003; Tully et al., 2003). Drugs boosting cAMP/CREB or preventing breakdown of cAMP are currently under investigation and are in clinical trials (Alberini et al., 2014). In this study, 17-AAG increased phosphor-CREB (ser133) and BDNF in cultured models and mouse brain, indicating a possible HSF1/cAMP/CREB/BDNF signaling pathway. Hsp90 inhibition of induced cAMP/CREB activation may present another potential target for memory improvement (Figure 6-1).

In addition to heat shock response and its induced heat shock proteins, this work looks to highlight HSF1-mediated transcriptional upregulation of multiple synaptic genes, including genes coding for synapsin I, synaptophysin, PSD95 and BDNF. We have also identified that HSF1 is capable of inducing several immediate early genes (IEGs), including Arc, c-Fos, early growth response protein 1 (Egr1), early growth response protein 2 (Egr2), early growth response protein 3 (Egr3), and early growth response protein 4 (Egr4), etc. 


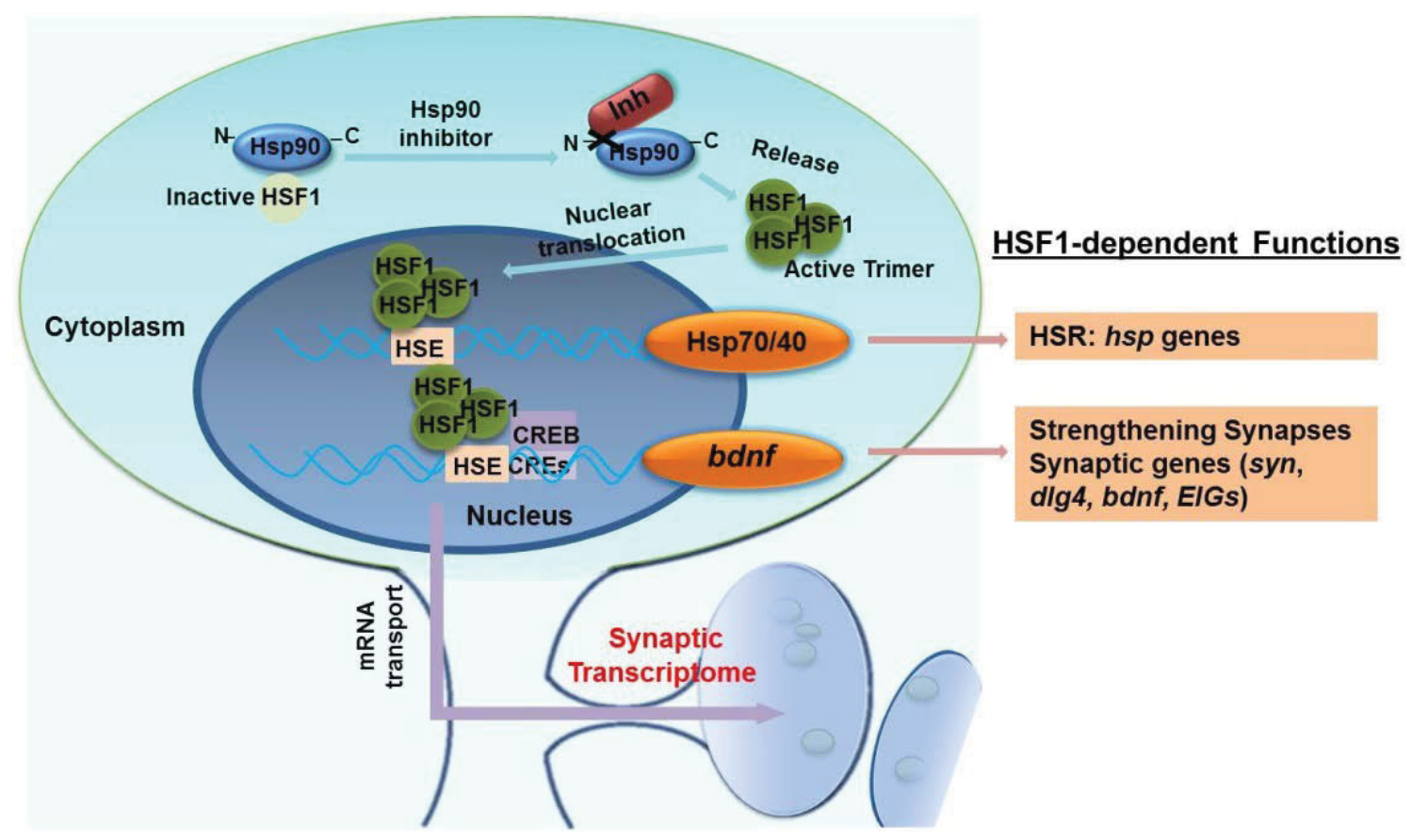

Figure 6-1 Schematic Illustration of HSF1-mediated Mechanism.

The cartoon illustrates how an Hsp90 inhibitor activates HSF1, ultimately leading to gene transcriptional activation of both neuronal HSPs and synaptic proteins. HSF1-mediated genes play major roles both in heat shock-like stress response (HSR) and in synaptic regulation. It is well-accepted that local translation machinery is required for learning and memory in dendrites. Despite a decade-long effort, there is still little known regarding the identity of the RNA molecules that are transported from the neuronal cell body (nucleus) to the synapse. Given the notion that heat shock responsive element (HSEs) are present in the promoters of several pre- and post-synaptic genes (syn I/synapsin I, Dlg4/PSD95, bdnf/BDNF, etc.), we speculate that HSF1 activation is required for the expression of these genes. Because the mRNAs of these genes are known to be transported to synapses and protein translated locally in dendrites, we therefore believe that HSF1 regulates a functional synaptic transcriptome. Activated CREB-P is known to be involved in this process. Both HSEs and CREs are present in many of the bdnf gene promoters, with some sites being close in vicinity or even overlapping. Therefore, HSF1 and CREB may interact in regulating expression. 
Analysis of mRNA transcription was conducted by co-workers in Dr. Liao's lab. Mice received foot shocks at an intensity of $0.7 \mathrm{~mA}$ twice per 1 min interval. $1 \mathrm{~h}, 4 \mathrm{~h}$ and 6 $\mathrm{h}$ time points were chosen post-foot shock to examine the transcriptional activity. Two brain regions, hippocampal CA1 and amygdala were collected for gene profiling analysis. Immunofluorescence staining revealed a rapid increase of nuclear HSF1 in hippocampal CA1 pyramidal neurons within the first hour and the activity was sustained for at least 6 hours. Immunofluorescence signal weakened at the $6 \mathrm{~h}$ time point, especially in comparison to the $1 \mathrm{~h}$ and $4 \mathrm{~h}$ time points. qRT-PCR demonstrated simultaneous upregulation of c-fos and Arc in hippocampal CA1 and, to a lesser degree, in the amygdala, and both peaked at $1 \mathrm{~h}$ post-foot shock training in mice. This was consistent with the increase of nuclear HSF1 post-foot shock exhibited in immunofluorescence staining. Different BDNF exon isoforms were also discovered to be elevated after foot shock, specifically the activity dependent exon I, and to a lesser extent exons IV and V (Lubin et al., 2012).

Consistent with foot shock stress, Hsp90 inhibition yielded similar results. Hsp90 inhibition was achieved by systemic administrating of OS47720 in mice. A complete gene profiling was conducted using a commercially available synaptic plasticity PCR array kit (Qiagen, Inc.). Array data confirmed the increase of Arc, c-Fos, and the important Dlg4 (coding gene for PSD95) upon OS47720 treatment. Several IEGs were also significantly upregulated, including Egr1, Egr2, Egr3, Egr4. A full gene profiling analysis revealed genomic transcriptional regulation of HSF1.

Structurally, based on the consensus sequences of HSF1-binding elements (HSEs), our work identified potential HSEs in the promoter regions of these genes. Among them, we provide evidence of direct binding of HSF1 to an HSE1 site in close proximity to the key CREB binding site (CRE) in bdnf promoter I, which was upregulated $1 \mathrm{~h}$ post-foot shock. Additionally, several IEGs were also induced at this time point. This result implies that HSF1 is another principle transcriptional factor required for gene upregulation during consolidation or retention of fear contextual conditioned freezing memory. This concept was also supported by the data demonstrating significant abrogating effects of the HSF1 inhibitor administered $1 \mathrm{~h}$ post-training (Figure 5-7).

The HSF1 regulated transcriptome was reported in some cases to be impaired during neurodegeneration. Indeed, Prahlad and Morimoto found that repressed HSF1 regulated transcriptional levels of heat shock proteins in neurodegenerative disease models in C. elegans (Prahlad and Morimoto, 2009). The HSF1-regulated transcriptome was also found to be blunted by an aggregated huntingtin (Htt) protein, which may participate in the neurodegeneration observed in HD (Riva et al., 2012).

Therefore, based on the findings from the current study together with findings from other labmates, we hypothesize that HSF1 plays a regulatory role in reshaping the synaptic transcriptome on a genomic level that ultimately contributes to neurodegeneration and potentially represents another master transcriptional factor for memory (Figure 6-1). In order to examine this hypothesis, systemic biological approaches such as ChIP-sequencing will be utilized to compare CREB and HSF1 on 
identifying the similarities of DNA binding sites. We predict that CREB and HSF1, both pertinent to synaptic functions, will have significant overlapping targeting genes.

\subsubsection{Neuronal Structure: Dendritic Spines}

Axons, dendrites and dendritic spines comprise the active neuronal network. A typical dendritic spine has a spine head and a spine neck that connect the head of the spine and the shaft of the dendrite. Dendritic spines are variable and "plastic" which allows them to maintain dynamic homeostasis. Dendritic spines with synapses undergo rapid and broad changes in number, size, and shape within the neuronal network.

Approximate $10-20 \%$ of spines can spontaneously form and be eliminated within hours. Due to the dynamic nature of the actin cytoskeleton in spines, most of the spines change their shape within seconds to several minutes. Spine dynamics are mostly activitydependent and BDNF is believed to be a key determinant in the levels of spines. In neuropsychiatric disorders, such as autism spectrum disorders (ASD), schizophrenia and $\mathrm{AD}$, abnormalities in dendritic spines, particularly the largely reduced number and ratio of immaturity of spines, may be the original insult that initiates the pathologic cascade (Penzes et al., 2011).

The close relation between structural dynamics of dendritic spines and cognitive memory has been extensively studied (Segal et al., 2005; Kasai et al., 2010). Increased spine formation and the enlargement of the spine head are believed to promote spatial learning and working memory (Mahmmound et al., 2015). It is not surprising since dendritic spines are the structural basis for functional activity. Thus, dynamic dendritic spines serve as a common substrate for neurodegenerative diseases with cognitive impairment.

The development of the two-photon excitation confocal microscopy greatly facilitates the study of the dynamics of dendritic spines and their correlation with functional outcomes, such as behaviors. By using it, high resolution imaging in living tissue becomes feasible. Transcranial two-photon microscopy was further developed for long-term imaging and tracing each individual dendritic spine in living animals (Grutzendler et al., 2002).

In the current study, through collaboration we also examined the dynamics of dendritic spines (spine formation and elimination) in young transgenic mice (postnatal 30-32 days) that express yellow fluorescent protein (YFP) in pyramidal neurons in specific cortical layer V region, before and after OS47720 treatment. We observed prominently enhanced spine formation $24-48 \mathrm{~h}(\sim 13 \%$ of increase) in cortical layer $\mathrm{V}$ following a single injection of OS47720 $(100 \mathrm{mg} / \mathrm{kg})$. The percentage of spine elimination was not changed. Although the hippocampal region would be a more ideal setting to investigate, it is unfeasible to get the probe to that deep area. Further investigation on spine formation will be necessary for Alzheimer's mouse models after chronic OS47720 treatment. 


\subsubsection{Neuronal Network Activity}

Neuronal network activity has long been recognized as the fundamental basis for all memory processes, including formation, storage, maintenance, and retrieval. LongTerm Potentiation (LTP) and Long-Term Depression (LTD) are among the most important neuronal events in spatial learning and memory observed in the hippocampus. The study on LTP began as early as the 1970s, where it was first discovered that rabbit hippocampus, when subjected to short high-frequency electrical stimulation (HFS) (for example, a few seconds), could enhance synaptic transmission for several days to weeks. The mechanism of LTP was studied due to the discovery of the unique properties of NMDA receptors primarily found in cultured hippocampal slices. NMDA receptor antagonists blocked the occurrence of LTP induced by HFS, but did not affect synaptic transmission induced by low-frequency stimulation. The calcium influx caused by the opening of NMDA channels by glutamate binding triggered LTP (Purves et al., 2001). Enhanced LTP is therefore believed to promote hippocampus-dependent learning and memory.

We examined the change of LTP in hippocampus after the treatment of 17-AAG on rat cultured brain slices. In this study, LTP in the DG region, instead of commonly investigated CA1-CA3 regions, was measured. The rationale of doing this was that synaptic plasticity in the DG region was severely impaired, while synaptic plasticity in the CA1 region was relatively spared in several FAD mouse models. A single administration of 17-AAG was given $30 \mathrm{~min}$ before the high-frequency stimulation (HFS). Significantly enhanced LTP recorded from the granule neuron synapses at rat medial perforant path-dentate gyrus (called perforant pathway) was observed. Both the amplitude of population spikes (PS) and the slope of excitatory postsynaptic potentials (EPSP) were significantly elevated after the treatment. On the other hand there were no obvious changes in basal levels of synaptic transmission without HFS was observed. When 17-AAG was given 30 min after the HFS, no change was found in either the amplitude of PS or the slope of EPSP. Therefore, the effect of 17-AAG is likely to be effective on the induction phase, but not the maintenance phase of hippocampal LTP (Chen et al., 2014).

Memory processes are based on neuronal communication and plasticity. Local field potential (LFP) indicates the neuronal network state and accounts for the neuronal activity response (Kelly et al., 2010). By computing the coherence, we can evaluate the correlation of local field potentials between two recording sites in different brain regions. Enhanced coherence, particularly in the low frequency oscillation (theta phase synchronization at 4-8 Hz for example), in the hippocampus (CA1 and CA3) was observed during the tasks involving working memory, while the coherence, in the delta $(0.5-3 \mathrm{~Hz})$, theta $(4-8 \mathrm{~Hz}), \beta(12-30 \mathrm{~Hz})$ and gamma $(30-100 \mathrm{~Hz})$ phases were enhanced between pre-frontal cortex and the hippocampus during the tasks involving long-term memory (Montgomery et al., 2007). These new findings implied that both working memory and long-term memory could be supported by coherence enhancement (phase synchronization), since these two forms of memories were traditionally considered separate systems. 
In the in vivo electrophysiology study of aged mice (18 months), OS47720 was found to mediate its effect via highly novel synaptic strengthening mechanisms via modulation of Hsp90. The unique sustained ( $>3 \mathrm{~d})$ induction of synaptic genes/protein expression after a single i.p. injection of OS47720 may be attributed to multiple mechanisms, including transcriptional regulation. This was not surprising given the large pool $(>800)$ of cellular proteins identified as Hsp90 client proteins, many of which may include membrane receptors necessary for neuronal transmission (e.g., AMPAR) (Gerges et al., 2004), as well as proteins regulating post-transcriptional microRNAs and translational processes.

In the current study, we demonstrated that, in healthy aged mice (18 months), OS47720 alters coherence of LFP activity within the hippocampal CA1 region and between CA1 and the prefrontal cortex for $\beta(12-30 \mathrm{~Hz})$, gamma $(30-100 \mathrm{~Hz})$ and higher frequency $(190-200 \mathrm{~Hz})$ oscillations. Coherence measures the degree of phaselocking of oscillatory neuronal activity between the two recording sites. Coherence is an indicator of neuronal communication between brain structures that has been implicated in several cognitive processes (Bastos et al., 2015; Buzsaki and Schomburg, 2015; Fries et al., 2015). Abnormalities in coherence (reduced) of brain activity have been shown to be associated with AD, both in humans (Chen et al., 2013; Hsiao et al., 2013) and in mouse models (Liu et al., 2013) of the disease. It has been proposed that measures of coherence of brain activity could serve as a biomarker for AD (Sankari et al., 2012; Basar et al., 2013). LFP coherence between the PFC and CA1 within the gamma frequency range has been strongly implicated in attention, working memory and memory consolidation (Spellman et al., 2015; Benchenane et al., 2011). Our findings suggest that the rescue of memory deficits by OS47720 treatment observed in AD mice is indicative of OS47720 affecting coherent neuronal activity within hippocampus, and between the PFC and CA1.

\subsubsection{Impaired HSF1 and Dysregulation in Neurodegeneration}

HSF1 dysregulation in neurodegeneration remains largely understudied. There was a recently published report claiming dysregulation of heat shock protein under conditions of pathological TDP-43 aggregation (ALS associated gene). In their research, Chang HY et al. found that 17-AAG reduced the aggregation levels of pathological misfolded TDP-43 protein by normalizing heat shock proteins, mainly HSF1, Hsp70 and Hsp27; however it was left unaddressed exactly how heat shock proteins had been dysregulated in in vitro cell culture model under those pathological conditions (Chang et al., 2013). Protein expression levels of HSF1 were significantly reduced in the granule Purkinje neurons in AD rat cerebellum. Protein overexpression by AAV-delivered HSF1 into the brain stem reversed neurodegeneration (Jiang et al., 2013).

For this study in aged Tg2576 mice, in both 3 months and 6 months treatment cohorts, we surprisingly found significantly reduced $(\sim 20 \%)$ protein expression of HSF1 in the hippocampi relative to their Non-Tg littermates (Figure 4-10 and Figure 4-12). Chronic OS47720 treatment not only rescued reduced HSF1 expression, but also elevated basal levels of expression in Non-Tg mice. We have evidence supporting the role of an 
Hsp90 inhibitor upregulating the hsf1 gene in neurons. The idea that a specific Hsp90 inhibitor can upregulate the HSF1 gene and regulate protein expression has not yet been reported proven.

According to our study, although soluble A $\beta$ can also induce HSF1 degradation in neuronal cellular cultures, as well as in APP-overexpressing FAD transgenic mice, the extent of HSF1 loss is not as severe as in cases of ALS, alpha-synuclein, or R6/2 Htt transgenic mice. This implied the existing common fact of HSF1 dysregulation and also different pathologies of each individual disease. Recently, other members from Dr. Liao's laboratory discovered that $\alpha$-synuclein overexpression lead to ubiquitination and further degradation of HSF1 via the ubiquitin proteasome system (UPS). This process is strictly mediated by the E3 ubiquitin-protein ligase NEDD4 also known as neural precursor cell expressed developmentally down-regulated protein 4 (NEDD4). Reduced NEDD4 or increased HSF1 via biochemical manipulation exhibited neuroprotection against $\alpha$ synuclein induced toxicity. In vivo studies confirmed and revealed loss of HSF1 protein due to UPS-mediated protein degradation in patient brains with Lewy's bodies. This was caused by proteotoxic stresses from misfolded and aggregated proteins such as $\alpha-$ synuclein, which may contribute to $\alpha$-synucleinopathy (Kim et al., 2015).

As a master transcriptional regulator in all mammalian cells, loss of HSF1 can cause many cellular and subcellular consequences, most of which would be harmful. As a result, HSF1-mediated heat shock response and stress response will be compromised. This will greatly impact the disease onset, development and progression during pathological neurodegeneration. Moreover, as an integrated part for keeping proteasomal homeostasis, even partial loss of HSF1 could lead to detrimental effect on protein degradation machinery system. Given the fact that, in our study HSF1 can not only masterly regulates its classic heat shock and stress responses, but also regulates a couple of genes associated with synaptic functions, such as bdnf, partial loss of HSF1 was found to significantly damage synaptic functions and its causal behavior outcomes (locomotor activity, object recognition memory, etc.) (Figure 5-5). Therefore due to the multifunctional role of HSF1, HSF1 dysregulation may represent a common and fundamental mechanism of neurodegenerative disease. It should be noted that the reduction of HSF1 caused by UPS degradation appeared to be induced only by proteotoxic stress, and not by heat stress, per our recent study. Hsp90 inhibitors induced HSF1 activation that may correct and normalize the dysregulated function of HSF1 and its mediated heat shock-like response and synaptic functions.

\subsubsection{Therapeutic Implication of OS47720 in AD}

Although HSF1 is well known as the most important master heat shock and stress response regulator, its role and regulatory mechanism in CNS disease remains unclear. This restudy was carried out to address this void in understanding, its role in neurodegenerative diseases. Upon investigating this Hsp90 inhibitor OS47720, we believe that pharmacological agents can activate and stabilize HSF1 protein expression 
and function with therapeutic promise in treating chronic neurodegenerative diseases, such as AD.

It has been over two decades since the concept of targeting Hsp90 first arose. Hsp90 has been validated in regulating several tumor suppressor pathways. Successful application of Hsp90 inhibition in various cancers in both preclinical and clinical studies will greatly support the on-going development of those agents. On the other hand, it has been approximate 10 years since the idea of using Hsp90 inhibitors in treating neurodegenerative diseases was first introduced. While there were several Hsp90 inhibitors exhibiting neuroprotection potential in in vitro models of many diseases, limited success was achieved with in vivo activity. Compared with the four currentlyknown CNS-permeable Hsp90 inhibitors (Dickey et al., 2007; Labbadia et al., 2011; Cha et al., 2014; Luo et al., 2007), which had in vivo testing in models of neurodegenerative diseases (Table 6-1), OS47720 is the only Hsp90 inhibitor compound that has demonstrated superb in vivo therapeutic performance, including an apparent lack of systemic toxicity at therapeutically-relevant dosage over a chronic period of time (3 months in presymptomatic mice and 6 months in middle symptomatic mice). There is no follow-up study for those four compounds, with possible reasons including long-term toxicity, lack of in vivo therapeutic efficacy, or the pathological differences among neurodegenerative diseases.

Currently there is limited success treating AD with CNS-permeable compounds. This leads us to consider a potential fundamental difference between AD and other neurodegenerative diseases (PD, HD, ALS, etc.). It appears that AD is more of a synaptic disease (Palop and Muche, 2010; Koffie et al., 2011), characterized by a slow and progressive deterioration, in comparison to those diseases caused by severe proteinopathy, which is more rapidly developed. The idea of applying Hsp90 inhibitors to AD becomes more attractive and promising when considering the twenty to thirty year window of chronic neurodegeneration in AD compared to a less than 2 year disease onset in ALS. Our work firstly presents a pharmacologically feasible Hsp90 inhibitor (OS47720) by demonstrating the feasibility of treating a chronic dysfunctional synaptic disease such as AD. Most notably, it is effective in treating early and middle symptomatic mice with amyloid plaque load via HSF1 mediated synaptic protection mechanism.

\subsection{Conclusions}

In this research, we used various biochemical and behavioral approaches to study Hsp90 inhibitors feasibility in AD treatment. Through the investigation of a commercially available Hsp90 inhibitor, 17-AAG, we proved that targeting Hsp90 inhibition was effective in preventing $A \beta$-induced toxicity, in both an in vitro cell culture model and an in vivo acute $A \beta$ mouse model. In addition to the regulation of traditional heat shock and stress responses, HSF1 efficacy in regulation of synaptic genes and functions ultimately championed clinical application. The novel compound OS47720 overcomes the two major developmental hurdles for Hsp90 inhibitors: systemic toxicity and limited CNS permeability. No noticeable systemic toxicity was observed based on 
body weight and daily activity monitoring during the chronic 3 months and 6 months treatment in two different Tg2576 mice cohorts, suggesting a feasible safety profile. A single intraperitoneal injection of OS47720 at $100 \mathrm{mg} / \mathrm{kg}$ dosage induced rapid compound accumulation in the brain within $1 \mathrm{~h}$, and was gradually cleared by $24 \mathrm{~h}$. Despite a short half-life of OS47720 in mouse brain, a single systemic injection induces rapid and long-lasting ( $>3 \mathrm{~d}$ ) nuclear activation of HSF1, suggesting good CNS permeability. We then demonstrated that chronic treatment with this proprietary compound OS47720 not only elicited a heat shock-like response, but also provided synaptic protection in early and middle symptomatic Tg2576 mice, a mouse model of Alzheimer's disease (AD), as evidenced by various behavioral and biochemical tests. Mechanistic studies indicated that the therapeutic effects of OS47720 depended upon HSF1 activation and the subsequent HSF1-mediated transcriptional events on synaptic genes. Hence, this work demonstrates promising translational significance. Taken together, this work reveals not only that the Hsp90 inhibitor OS47720 holds therapeutic promise in treating AD, but also identifies that HSF1 plays an instrumental role in synaptic function and memory by way of synaptic transcriptome modulation.

\subsection{Future Directions}

Hsp90 inhibitors have been recognized as promising agents in treating various types of cancers for over two decades. Previous and current studies (Dickey et al., 2007; Labbadia et al., 2011; Cha et al., 2014; Luo et al., 2007) have brought neurodegenerative diseases to the forefront of diseases that Hsp90 inhibitors can potentially treat. Our work validated the feasibility of using one type of Hsp90 inhibitor - OS47720 in treating AD characterized by synaptic dysfunction. There was only a single AD mouse model used in this study, thus it will be necessary to test OS47720 in additional AD mouse models, including APP models (J20, 5×FAD, etc.) and tau models (PS19, rTg4510, etc.), for its in vivo efficacy. Tau pathology represents the other molecular hallmark of AD.

In our current study, post-mortem tests revealed that OS47720 demonstrated a therapeutic effect on synapse protection without affecting $\mathrm{A} \beta$ production and $\mathrm{A} \beta$ plaque removal. Since $A \beta$ has been well characterized for its cellular toxicity, it may be worth investigating as a target, perhaps being treated with a combination of an Hsp90 inhibitor and some form of anti-amyloid treatment (e.g., $\beta$-secretase inhibitors). This may ultimately offer a viable treatment paradigm for treating symptomatic AD patients.

Currently there are three "viable" Hsp90 inhibition compounds that are actively being tested for their potential in vivo efficacy in treating neurodegenerative diseases. Among them, The NVP series compounds from Novartis Oncology company exhibits the greatest efficacy with regard to Hsp90 inhibition, with IC50 NVP-Hsp990=0.6 nM and IC50NVP-AUY922= $13 \mathrm{nM}$. These compounds are commercially available. The AU series compounds are synthesized at Dr. Chiosis's laboratory from Memorial SloanKettering Cancer Center. The leading compounds PU-H71 and PU-DZ8 have modest potency on Hsp90 inhibition, with IC50PU-H71= $80 \mathrm{nM}$ and IC50PU-DZ8 $=160 \mathrm{nM}$ (Taldone et al., 2010). Similarly, OS47720 displays an IC50 value at around a hundred 
nanomolar level. Testing the more potent compound AUY922 becomes feasible in further addressing the hypothesis of targeting Hsp90 inhibition for treating AD through HSF1mediated regulation at synapses. So far, most of compound development has been focused on targeting the ATP binding pocket located in the N-terminus domain of Hsp90. The other C-terminus domain could be a potential secondary target site; however there is minimal research to justify this. Coumarin antibiotics, such as novobiocin, are the prototypic inhibitors targeting this site which still requires future work.

Although significant progress has been made in current study, many important questions remain unanswered. This project has demonstrated that the OS47720 compound represents a useful pharmacological inducer of heat shock response, and further serves as a promoter of synaptic activity with prospective in vivo application. 


\section{LIST OF REFERENCES}

Alberini CM, Kandel ER. (2014). The regulation of transcription in memory consolidation. Cold Spring Harb Perspect Biol. 7, a021741.

Allen B, Ingram E, Takao M, Smith MJ, Jakes R, Virdee K, Yoshida H, Holzer M, Craxton M, Emson PC, et al. (2002). Abundant tau filaments and nonapoptotic neurodegeneration in transgenic mice expressing human P301S tau protein. J Neurosci. 22, 9340-9351.

Alzheimer's associtation. 2015 Alzheimer's disease facts and figures. Viewed 122016 January. $<$ http://www.alz.org/facts/downloads/facts_figures_2015.pdf $>$.

Amin K, Ip C, Jimenez L, Tyson C, Behrsing H. (2005). In vitro detection of differential and cell-specific hepatobiliary toxicity induced by geldanamycin and 17allylaminogeldanamycin using dog liver slices. Toxicol Sci. 87, 442-450.

Ammirante M, Rosati A, Gentilella A, Festa M, Petrella A, Marzullo L, Pascale M, Belisario MA, Leone A, Turco MC. (2008). The activity of hsp90 alpha promoter is regulated by NF-kappa B transcription factors. Oncogene. 27; 1175-1178.

Amor S, Peferoen LA, Vogel DY, Breur M, van der Valk P, Baker D, van Noort JM. (2014). Inflammation in neurodegenerative diseases--an update. Immunology. 142, 151166.

Amor S, Puentes F, Baker D, van der Valk P. (2010). Inflammation in neurodegenerative diseases. Immunology. 129, 154-169.

Andorfer C, Kress Y, Espinoza M, de Silva R, Tucker KL, Barde YA, Duff K, Davies P. (2003). Hyperphosphorylation and aggregation of tau in mice expressing normal human tau isoforms. J Neurochem. 86, 582-590.

Ansar S, Burlison JA, Hadden MK, Yu X, Desino KE, Bean J, Neckers L, Audus KL, Michaelis ML, Blagg BS. (2007). A non-toxic Hsp90 inhibitor protects neurons from A $\beta$ induced toxicity. Bioorg Med Chem Lett. 17, 1984-1990.

Arrasate M, Finkbeiner S. (2012) Protein aggregates in Huntington's disease. Exp Neurol. $238,1-11$.

Auluck PK, Chan HY, Trojanowski JQ, Lee VM, Bonini NM. (2002). Chaperone suppression of alpha-synuclein toxicity in a Drosophila model for Parkinson's disease. Science. 295, 865-868.

Banerji U, O'Donnell A, Scurr M, Pacey S, Stapleton S, Asad Y, Simmons L, Maloney A, Raynaud F, Campbell M, et al. (2005). Phase I pharmacokinetic and 
pharmacodynamic study of 17-allylamino, 17-demethoxygeldanamycin in patients with advanced malignancies. J Clin Oncol. 23, 4152-4161.

Barco A, Pittenger C, Kandel ER. (2003). CREB, memory enhancement and the treatment of memory disorders: promises, pitfalls and prospects. Expert Opin Ther Targets. 7, 101-114.

Barluenga S, Fontaine JG, Wang C, Aouadi K, Chen R, Beebe K, Neckers L, Winssinger N. (2009). Inhibition of Hsp90 with pochoximes: SAR and structure-based insights. Chembiochem. 10, 2753-2759.

Baruch-Suchodolsky R, Fischer B. (2009). "A $\beta 40$, either soluble or aggregated, is a remarkably potent antioxidant in cell-free oxidative systems". Biochemistry. 48, 43544370 .

Basar E, Basar-Eroglu C, Guntekin B and Yener GG. (2013). Brain's alpha, $\beta$, gamma, delta, and theta oscillations in neuropsychiatric diseases: proposal for biomarker strategies. Suppl Clin Neurophysiol. 62, 19-54.

Bastos AM, Vezoli J, Bosman CA, Schoffelen JM, Oostenveld R, Dowdall JR, De Weerd P, Kennedy H, Fries P. (2015). Visual areas exert feedforward and feedback influences through distinct frequency channels. Neuron. 85, 390-401.

Batulan Z, Shinder GA, Minotti S, He BP, Doroudchi MM, Nalbantoglu J, Strong MJ, Durham HD. (2003). High threshold for induction of the stress response in motor neurons is associated with failure to activate HSF1. J Neurosci. 23, 5789-5798.

Batulan Z, Taylor DM, Aarons RJ, Minotti S, Doroudchi MM, Nalbantoglu J, Durham HD. (2006). Induction of multiple heat shock proteins and neuroprotection in a primary culture model of familial amyotrophic lateral sclerosis. Neurobiol Dis. 24, 213-225.

Becker RE, Greig NH and Giacobini E. (2008). Why do so many drugs for Alzheimer's disease fail in development? Time for new methods and new practices? J. Alzheimers Dis. $15,303-325$.

Benchenane K, Tiesinga PH and Battaglia FP. (2011). Oscillations in the prefrontal cortex: a gateway to memory and attention. Curr Opin Neurobiol. 21, 475-485. Bertoni-Freddari C, Fattoretti P, Meier-Ruge W, Ulrich J. (1989). Computer-assisted morphometry of synaptic plasticity during aging and dementia. Pathol Res Pract. 185, 799-802.

Bertram L, Tanzi RE. (2005). The genetic epidemiology of neurodegenerative disease. J Clin Invest. 115, 1449-1457.

Blagosklonny MV, Toretsky J, Neckers L. (1995). Geldanamycin selectively destabilizes and conformationally alters mutated p53. Oncogene. 11, 933-939. 
Bobkova NV, Garbuz DG, Nesterova I, Medvinskaya N, Samokhin A, Alexandrova I, Yashin V, Karpov V, Kukharsky MS, Ninkina NN, et al. (2014). Therapeutic effect of exogenous hsp70 in mouse models of Alzheimer's disease. J. Alzheimers Dis. 38, 425435.

Borchelt DR, Davis J, Fischer M, Lee MK, Slunt HH, Ratovitsky T, Regard J, Copeland NG, Jenkins NA, Sisodia SS, et al. (1996). A vector for expressing foreign genes in the brains and hearts of transgenic mice. Genet Anal. 13, 159-163.

Brincat SL, Miller EK. (2015). Frequency-specific hippocampal-prefrontal interactions during associative learning. Nat Neurosci. 18, 576-581.

Brown IR. (2007). Heat shock proteins and protection of the nervous system. Ann N Y Acad Sci. 1113, 147-158.

Brown RC, Lockwood AH, Sonawane BR. (2005). Neurodegenerative diseases: an overview of environmental risk factors. Environ Health Perspect. 113, 1250-1256.

Buzsaki G and Schomburg EW. (2015). What does gamma coherence tell us about interregional neural communication? Nat Neurosci. 18, 484-489.

Buzsáki G, Buhl DL, Harris KD, Csicsvari J, Czéh B, Morozov A. (2003). Hippocampal network patterns of activity in the mouse. Neuroscience. 116, 201-211.

Cajal Ry. (1991). Cajal's Degeneration and regeneration of the nervous system. Oxford Press, London.

Carmichael J, Chatellier J, Woolfson A, Milstein C, Fersht AR, Rubinsztein DC. (2000). Bacterial and yeast chaperones reduce both aggregate formation and cell death in mammalian cell models of Huntington's disease. Proc Natl Acad Sci USA. 97, 97019705.

Carnemolla A, Lazell H, Moussaoui S and Bates GP. (2015). In vivo profiling reveals a competent heat shock response in adult neurons: implications for neurodegenerative disorders. PLoS One 10, e0131985.

Casas C, Sergeant N, Itier JM, Blanchard V, Wirths O, van der Kolk N, Vingtdeux V, van de Steeg E, Ret G, Canton T, et al. (2004). Massive CA1/2 neuronal loss with intraneuronal and $\mathrm{N}$-terminal truncated Abeta42 accumulation in a novel Alzheimer transgenic model. Am J Pathol 165, 1289-1300.

Cavanaugh SE, Pippin JJ, Barnard ND. (2014). Animal models of Alzheimer disease: historical pitfalls and a path forward. ALTEX. 31, 279-302.

Cha JR, St Louis KJ, Tradewell ML, Gentil BJ, Minotti S, Jaffer ZM, Chen R, Rubenstein AE, Durham HD. (2014). A novel small molecule Hsp90 inhibitor, OS47720 
30001, differentially induces heat shock proteins in nervous tissue in culture and in vivo. Cell Stress Chaperon. 19, 421-435.

Chang HY, Hou SC, Way TD, Wong CH, Wang IF. (2013). Heat-shock protein dysregulation is associated with functional and pathological TDP-43 aggregation. Nat. Commun. 4, 2757.

Chartier-Harlin MC, Crawford F, Houlden H, Warren A, Hughes D, Fidani L, Goate A, Rossor M, Roques P, Hardy J, et al. (1991). Early-onset Alzheimer's disease caused by mutations at codon 717 of the beta-amyloid precursor protein gene. Nature 353, 844-846.

Chasseigneaux S, Allinquant B. (2012). Functions of A $\beta$, sAPP $\alpha$ and sAPP $\beta$ : similarities and differences. J Neurochem. 120, 99-108.

Chen CC, Hsu CY, Chiu HW, Hu CJ, Lee TC. (2013). Frequency power and coherence of electroencephalography are correlated with the severity of Alzheimer's disease: A multicenter analysis in Taiwan. J Formos Med Assoc. 114, 729-735.

Chen Y, Wang B, Liu D, Li JJ, Xue Y, Sakata K, Zhu LQ, Heldt SA, Xu H, Liao FF. (2014). Hsp90 chaperone inhibitor 17-AAG attenuates A $\beta$-induced synaptic toxicity and memory impairment. J Neurosci. 34, 2464-2470.

Cheng IH, Palop JJ, Esposito LA, Bien-Ly N, Yan F, Mucke L. (2004). Aggressive amyloidosis in mice expressing human amyloid peptides with the Arctic mutation. Nat Med. 10, 1190-1192.

Chishti MA, Yang DS, Janus C, Phinney AL, Horne P, Pearson J, Strome R, Zuker N, Loukides J, French J, Turner S, et al. (2001). Early-onset amyloid deposition and cognitive deficits in transgenic mice expressing a double mutant form of amyloid precursor protein 695. J Biol Chem. 276, 21562-21570.

Dauer W and Przedborski S. (2003) Parkinson's disease: mechanisms and models. Neuron 39, 889-909.

Davies CA, Mann DM, Sumpter PQ, Yates PO. (1987). A quantitative morphometric analysis of the neuronal and synaptic content of the frontal and temporal cortex in patients with Alzheimer's disease. J Neurol Sci. 78, 151-164.

De Felice FG, Vieira MN, Bomfim TR, Decker H, Velasco PT, Lambert MP, Viola KL, Zhao WQ, Ferreira ST, Klein WL. (2009). Protection of synapses against Alzheimer'slinked toxins: insulin signaling prevents the pathogenic binding of Abeta oligomers. Proc Natl Acad Sci USA. 106, 1971-1976.

de la Rosa X, Santalucía T, Fortin PY, Purroy J, Calvo M, Salas-Perdomo A, Justicia C, Couillaud F, Planas AM. (2013). In vivo imaging of induction of heat-shock protein-70 gene expression with fluorescence reflectance imaging and intravital confocal 
microscopy following brain ischaemia in reporter mice. Eur J Nucl Med Mol Imaging. 40, 426-438.

DeBoer C, Meulman PA, Wnuk RJ, Peterson DH. (1970). Geldanamycin, a new antibiotic. J Antibiot (Tokyo). 23, 442-447.

DeKosky ST, Scheff SW. (1990). Synapse loss in frontal cortex biopsies in Alzheimer's disease: correlation with cognitive severity. Ann Neurol. 27, 457-464.

Delmotte P, Delmottte-Plauqe J. (1953). A new antifungal substance of fungal origin. Nature. 171, 344.

Dickey CA, Kamal A, Lundgren K, Klosak N, Bailey RM, Dunmore J, Ash P, Shoraka S, Zlatkovic J, Eckman CB, et al. (2007). The high-affinity Hsp90-CHIP complex recognizes and selectively degrades phosphorylated tau client proteins. J Clin Invest. 117, 648-658.

Eckman CB, Mehta ND, Crook R, Perez-tur J, Prihar G, Pfeiffer E, Graff-Radford N, Hinder P, Yager D, Zenk B, et al. (1997). A new pathogenic mutation in the APP gene (I716V) increases the relative proportion of A $\beta 42(43)$. Hum Mol Genet. 6, 2087-2089. Evans CG, Chang L, Gestwicki JE. (2010). Heat shock protein 70 (hsp70) as an emerging drug target. J Med Chem. 53, 4585-4602.

Falsone SF, Kungl AJ, Rek A, Cappai R, Zangger K. (2009). The molecular chaperone Hsp90 modulates intermediate steps of amyloid assembly of the Parkinson-related protein alpha-synuclein. J Biol Chem. 284, 31190-31199.

Fan GC. (2012). Chapter 14 - Role of Heat Shock Proteins in Stem Cell Behavior. Progress in Molecular Biology and Translational Science. 111, 305-322 Fernandez-Funez P., Rincon-Limas DE. (2014). Launching Hsp70 neuroprotection: two drugs better than one. Cell Cycle. 13, 1657-1658.

Ferrarini M, Heltai S, Zocchi MR, Rugarli C. (1992). Unusual expression and localization of heat-shock proteins in human tumor cells. Int J Cancer 51, 613-619. Fries P. (2015). Rhythms for Cognition: Communication through Coherence. Neuron 88, 220-235.

Games D, Adams D, Alessandrini R, Barbour R, Berthelette P, Blackwell C, Carr T, Clemens J, Donaldson T, Gillespie F, et al. (1995). Alzheimer-type neuropathology in transgenic mice overexpressing V717F beta-amyloid precursor protein. Nature 373, 523527.

Gandy S. (2005) The role of cerebral amyloid beta accumulation in common forms of Alzheimer disease. J Clin Invest. 115, 1121-1129. 
Gerges NZ, Tran IC, Backos DS, Harrell JM, Chinkers M, Pratt WB, Esteban JA. (2004). Independent functions of hsp90 in neurotransmitter release and in the continuous synaptic cycling of AMPA receptors. J. Neurosci. 24, 4758-4766.

Glenner GG, Wong CW. (1984). Alzheimer's disease and Down's syndrome: sharing of a unique cerebrovascular amyloid fibril protein. Biochem Biophys Res Commun. 122, 1131-1135.

Goate A, Chartier-Harlin MC, Mullan M, Brown J, Crawford F, Fidani L, Giuffra L, Haynes A, Irving N, James L, et al. (1991). Segregation of a missense mutation in the amyloid precursor protein gene with familial Alzheimer's disease. Nature. 349, 704-706.

Goetz MP, Toft D, Reid J, Ames M, Stensgard B, Safgren S, Adjei AA, Sloan J, Atherton $\mathrm{P}$, Vasile V, et al. (2005). Phase I trial of 17-allylamino-17-demethoxygeldanamycin in patients with advanced cancer. J Clin Oncol. 23, 1078-1087.

Gonsalves SE, Moses AM, Razak Z, Robert F, Westwood JT. (2011). Whole-genome analysis reveals that active heat shock factor binding sites are mostly associated with non-heat shock genes in Drosophila melanogaster. PLoS One 6, e15934.

Gorska M, Popowska U, Sielicka-Dudzin A, Kuban-Jankowska A, Sawczuk W, Knap N, Cicero G, Wozniak F. (2012). Geldanamycin and its derivatives as Hsp90 inhibitors. Front Biosci (Landmark Ed). 17, 2269-2277.

Gregory JM, Barros TP, Meehan S, Dobson CM, Luheshi LM. (2012) The aggregation and neurotoxicity of TDP-43 and its ALS-associated $25 \mathrm{kDa}$ fragment are differentially affected by molecular chaperones in Drosophila. PLoS One. 7: e31899.

Grem JL, Morrison G, Guo XD, Agnew E, Takimoto CH, Thomas R, Szabo E, Grochow L, Grollman F, Hamilton JM, et al. (2005). Phase I and pharmacologic study of 17(allylamino)-17-demethoxygeldanamycin in adult patients with solid tumors. J Clin Oncol. 23, 1885-1893.

Grutzendler J, Kasthuri N, Gan WB. (2002). Long-term dendritic spine stability in the adult cortex. Nature. 420, 812-816.

Haass C and Selkoe DJ. (2007). Soluble protein oligomers in neurodegeneration: lessons from the Alzheimer's amyloid beta-peptide. Nat Rev Mol Cell Biol. 8, 101-112. Hahn JS, Hu Z, Thiele DJ, Iyer VR. (2004). Genome-wide analysis of the biology of stress responses through heat shock transcription factor. Mol Cell Biol. 24, 5249-5256.

Hall A, Roberson E. (2012). Mouse models of Alzheimer's disease. Brain Res Bulletin. $88,3-12$. 
Hashimoto M, Rockenstein E, Crews L, Masliah E. (2003). Role of protein aggregation in mitochondrial dysfunction and neurodegeneration in Alzheimer's and Parkinson's diseases. Neuromolecular Med. 4, 21-36.

Hay DG, Sathasivam K, Tobaben S, Stahl B, Marber M, Mestril R, Mahal A, Smith DL, Woodman B, Bates GP. (2004). Progressive decrease in chaperone protein levels in a mouse model of Huntington's disease and induction of stress proteins as a therapeutic approach. Hum Mol Genet. 13, 1389-1405.

Heath EI, Hillman DW, Vaishampayan U, Sheng S, Sarkar F, Harper F, Gaskins M, Pitot HC, Tan W, Ivy SP, et al. (2008). A phase II trial of 17-allylamino-17-

demethoxygeldanamycin in patients with hormone-refractory metastatic prostate cancer. Clin Cancer Res. 14, 7940-7946.

Hebert LE, Weuve J, Scherr PA, Evans DA. (2013). Alzheimer disease in the United States (2010-2050) estimated using the 2010 Census. Neurology. 80, 1778-1783.

Heneka MT, Carson MJ, El Khoury J, Landreth GE, Brosseron F, Feinstein DL, Jacobs AH, Wyss-Coray T, Vitorica J, Ransohoff RM, et al. (2015). Neuroinflammation in Alzheimer's disease. Lancet Neurol. 14, 388-405.

Herzig MC, Winkler DT, Burgermeister P, Pfeifer M, Kohler E, Schmidt SD, Danner S, Abramowski D, Stürchler-Pierrat C, Bürki K, et al. (2004). Abeta is targeted to the vasculature in a mouse model of hereditary cerebral hemorrhage with amyloidosis. Nat Neurosci. 7, 954-960.

Hiltunen M, van Groen T, Jolkkonen J. (2009). "Functional roles of amyloid-beta protein precursor and amyloid-beta peptides: evidence from experimental studies". Journal of Alzheimer's Disease 18, 401-412.

Hindle JV. (2010). Ageing, neurodegeneration and Parkinson's disease. Age Ageing, 39, $156-161$.

Holcomb L, Gordon MN, McGowan E, Yu X, Benkovic S, Jantzen P, Wright K, Saad I, Mueller R, Morgan D, et al. (1998). Accelerated Alzheimer-type phenotype in transgenic mice carrying both mutant amyloid precursor protein and presenilin 1 transgenes. Nat Med. 4, 97-100.

Honig LS. (2012). Translational research in neurology: dementia. Arch. Neurol. 69, 969977.

Hoshino T, Murao N, Namba T, Takehara M, Adachi H, Katsuno M, Sobue G, Matsushima T, Suzuki T, Mizushima T. (2011). Suppression of Alzheimer's diseaserelated phenotypes by expression of heat shock protein 70 in mice. J Neurosci. 31, 52255234. 
Hsia AY, Masliah E, McConlogue L, Yu GQ, Tatsuno G, Hu K, Kholodenko D, Malenka RC, Nicoll RA, Mucke L. (1999). Plaque-independent disruption of neural circuits in Alzheimer's disease mouse models. Proc Natl Acad Sci USA. 96, 3228-3233.

Hsiao FJ, Wang YJ, Yan SH, Chen WT, Lin YY. (2013). Altered oscillation and synchronization of default-mode network activity in mild Alzheimer's disease compared to mild cognitive impairment: an electrophysiological study. PLoS One 8, e68792.

Hsiao K, Chapman P, Nilsen S, Eckman C, Harigaya Y, Younkin S, Yang F, Cole G. (1996) Correlative memory deficits, $A \beta$ elevation, and amyloid plaques in transgenic mice. Science. 274, 99-102.

Hu Y, Qu L, Schikorski T. (2008) Mean synaptic vesicle size varies among individual excitatory hippocampal synapses. Synapse. 62, 953-957.

Ikuina Y, Amishiro N, Miyata M, Narumi H, Ogawa H, Akiyama T, Shiotsu Y, Akinaga S, Murakata C. (2003). Synthesis and antitumor activity of novel Ocarbamoylmethyloxime derivatives of radicicol. J Med Chem. 46, 2534-2541.

Iqbal K, Alonso Adel C, Chen S, Chohan MO, El-Akkad E, Gong CX, Khatoon S, Li B, Liu F, Rahman A, et al. (2005). Tau pathology in Alzheimer disease and other tauopathies. Biochim Biophys Acta. 1739, 198-210.

Irvine GB, El-Agnaf OM, Shankar GM, Walsh DM. (2008). Protein aggregation in the brain: the molecular basis for Alzheimer's and Parkinson's diseases. Mol Med. 14, 451464.

Jackson T, Ramaswami M. (2003). Prospects of memory-modifying drugs that target the CREB pathway. Curr Opin Drug Discov Devel. 6, 712-719.

Jhaveri K, Modi S. (2012). Hsp90 inhibitors for cancer therapy and overcoming drug resistance. Adv Pharmacol. 65, 471-517.

Jiang YQ, Wang XL, Cao XH, Ye ZY, Li L, Cai WQ. (2013). Increased heat shock transcription factor 1 in the cerebellum reverses the deficiency of Purkinje cells in Alzheimer's disease. Brain Res. 1519, 105-111.

Jilani K, Qadri SM, Lang F. (2013). Geldanamycin-induced phosphatidylserine translocation in the erythrocyte membrane. Cell Physiol Biochem. 32, 1600-1609.

Jinwal UK, Abisambra JF, Zhang J, Dharia S, O'Leary JC, Patel T, Braswell K, Jani T, Gestwicki JE, Dickey CA. (2012). Cdc37/Hsp90 protein complex disruption triggers an autophagic clearance cascade for TDP-43 protein. J Biol Chem. 287, 24814-24820. 
Jung AE, Fitzsimons HL, Bland RJ, During MJ, Young D. (2008). Hsp70 and constitutively active HSF1 mediate protection against CDCrel-1-mediated toxicity. Mol Ther. 16, 1048-55.

Kalmar B, Burnstock G, Vrbová G, Urbanics R, Csermely P, Greensmith L. (2002). Upregulation of heat shock proteins rescues motoneurones from axotomy-induced cell death in neonatal rats. Exp Neurol. 176, 87-97.

Kalmar B, Greensmith L, Malcangio M, McMahon SB, Csermely P, Burnstock G. (2003). The effect of treatment with BRX-220, a co-inducer of heat shock proteins, on sensory fibers of the rat following peripheral nerve injury. Exp Neurol. 184, 636-647 Kamal A, Boehm MF, Burrows FJ. (2004). Therapeutic and diagnostic implications of Hsp90 activation. Trends Mol Med. 10, 283-290.

Kasai H, Fukuda M, Watanabe S, Hayashi-Takagi A, Noguchi J. (2010). Structural dynamics of dendritic spines in memory and cognition. Trends Neurosci. 33, 121-129. Katsuse O, Lin WL, Lewis J, Hutton ML, and Dickson DW. (2006). Neurofibrillary tangle-related synaptic alterations of spinal motor neurons of P301L tau transgenic mice. Neurosci Lett. 409, 95-99.

Kayed R, Head E, Thompson JL, McIntire TM, Milton SC, Cotman CW, Glabe CG. (2005). Common structure of soluble amyloid oligomers implies common mechanism of pathogenesis. Science. 300,486-489.

Kelly RC, Smith MA, Kass RE, Lee TS. (2010). Local field potentials indicate network state and account for neuronal response variability. J Comput Neurosci. 29, 567-579.

Kerman A, Chakrabartty A. (2011). Chapter 8 Protein Misfolding and Toxicity in Amyotrophic Lateral Sclerosis. Book: Non-fibrillar Amyloidogenic Protein Assemblies Common Cytotoxins Underlying Degenerative Diseases. 8, 257-288.

Kieran D, Kalmar B, Dick JR, Riddoch-Contreras J, Burnstock G, Greensmith L. (2004). Treatment with arimoclomol, a coinducer of heat shock proteins, delays disease progression in ALS mice. Nat Med. 10, 402-405.

Kim E, Wang B, Sastry N, Masliah E, Nelson PT, Cai H, Liao FF. (2016). NEDD4mediated HSF1 degradation underlies $\alpha$-synucleinopathy. Hum Mol Genet. 25, 211-222. Kittelberger KA, Piazza F, Tesco G, Reijmers LG. (2012). Natural amyloid- $\beta$ oligomers acutely impair the formation of a contextual fear memory in mice. PLoS One. 7, e29940.

Klyubin I, Walsh DM, Lemere CA, Cullen WK, Shankar GM, Betts V, Spooner ET, Jiang L, Anwyl R, Selkoe DJ. (2005). Amyloid $\beta$ protein immunotherapy neutralizes A $\beta$ oligomers that disrupt synaptic plasticity in vivo. Nat Med. 11, 556-561. 
Ko HS, Bailey R, Smith WW, Liu Z, Shin JH, Lee YI, Zhang YJ, Jiang H, Ross CA, Moore DJ, et al. (2009). CHIP regulates leucine-rich repeat kinase-2 ubiquitination, degradation, and toxicity. Proc Natl Acad Sci USA 106, 2897-2902.

Koffie RM, Hyman BT, Spires-Jones TL. (2011). Alzheimer's disease: synapses gone cold. Mol. Neurodegener. 6, 63.

Kremer B, Goldberg P, Andrew SE, Theilmann J, Telenius H, Zeisler J, Squitieri F, Lin B, Bassett A, Almqvist E, et al. (1994). A worldwide study of the Huntington's disease mutation. The sensitivity and specificity of measuring CAG repeats. N Engl J Med. 330, 1401-1406.

Labbadia J, Novoselov SS, Bett JS, Weiss A, Paganetti P, Bates GP, Cheetham ME. (2012). Suppression of protein aggregation by chaperone modification of high molecular weight complexes. Brain. 135, 1180-1196.

Laferla FM, Green KN, Oddo S. (2007). Intracellular amyloid-beta in Alzheimer's disease. Nat Rev Neurosci. 8, 499-509.

Langui D, Girardot N, El Hachimi KH, Allinquant B, Blanchard V, Pradier L, Duyckaerts C. (2004). Subcellular topography of neuronal Abeta peptide in APPxPS1 transgenic mice. Am J Pathol 165, 1465-1477.

Lanneau D, Brunet M, Frisan E, Solary E, Fontenay M, Garrido C. (2008). Heat shock proteins: essential proteins for apoptosis regulation. J Cell Mol Med. 12, 743-61.

Levites Y, Amit T, Mandel S, Youdim MB. (2003). Neuroprotection and neurorescue against Abeta toxicity and PKC-dependent release of nonamyloidogenic soluble precursor protein by green tea polyphenol (-)-epigallocatechin-3-gallate. FASEB J. 17, $952-954$.

Lewis J, McGowan E, Rockwood J, Melrose H, Nacharaju P, Van Slegtenhorst M, Gwinn-Hardy K, Paul Murphy M, Baker M, Yu X. (2000) Neurofibrillary tangles, amyotrophy and progressive motor disturbance in mice expressing mutant (P301L) tau protein. Nat Genet. 25, 402-405.

Li J, Buchner J. Structure, Function and Regulation of the Hsp90 Machinery. (2013). Biomed J. 36, 106-117.

Li JJ., Dolios G., Wang R., Liao FF. (2014). Soluble B-Amyloid Peptides, but Not Insoluble Fibrils, Have Specific Effect on Neuronal MicroRNA Expression. PLoS One. 9, e90770.

Li LH, Clark TD, Cowie CH, Rinehart KL Jr. (1977). Effects of geldanamycin and its derivatives on RNA-directed DNA polymerase and infectivity of Rauscher leukemia virus. Cancer Treat Rep. 61, 815-824. 
Li S, Hong S, Shepardson NE, Walsh DM, Shankar GM, Selkoe D. (2009). Soluble oligomers of amyloid $\beta$ protein facilitate hippocampal long-term depression by disrupting neuronal glutamate uptake. Neuron. 62, 788-801.

Liu Q, Li A, Gong L, Zhang L, Wu N, Xu F. (2013). Decreased coherence between the two olfactory bulbs in Alzheimer's disease model mice. Neurosci Lett. 545, 81-85.

Lu Y, Ansar S, Michaelis ML, Blagg BS. (2009). Neuroprotective activity and evaluation of Hsp90 inhibitors in an immortalized neuronal cell line. Bioorg Med Chem. 17, 17091715.

Lubin FD, Roth TL, Sweatt JD. (2008). Epigenetic regulation of BDNF gene transcription in the consolidation of fear memory. J Neurosci. 28, 10576-10586.

Luo W, Dou F, Rodina A, Chip S, Kim J, Zhao Q, Moulick K, Aguirre J, Wu N, Greengard P, et al. (2007). Roles of heat-shock protein 90 in maintaining and facilitating the neurodegenerative phenotype in tauopathies. Proc Natl Acad Sci USA. 104, 95119516.

Luo W, Sun W, Taldone T, Rodina A, Chiosis G. (2010). Heat shock protein 90 in neurodegenerative diseases. Mol Neurodegener. 5, 24.

Lynch G, Palmer LC, Gall CM. (2011). The likelihood of cognitive enhancement. Pharmacol Biochem Behav. 99, 116-129.

Lynch G. (2002). Memory enhancement: the search for mechanism-based drugs. Nat Neurosci. 5, 1035-1038.

Magrané J, Smith RC, Walsh K, Querfurth HW. (2004). Heat shock protein 70 participates in the neuroprotective response to intracellularly expressed $\beta$-amyloid in neurons. J Neurosci. 24, 1700-1706.

Mahmmoud RR, Sase S, Aher YD, Sase A, Gröger M, Mokhtar M, Höger H, Lubec G. (2015). Spatial and Working Memory Is Linked to Spine Density and Mushroom Spines. PLoS One. 10, e0139739.

Marcuccilli CJ, Mathur SK, Morimoto RI, Miller RJ. (1996). Regulatory differences in the stress response of hippocampal neurons and glial cells after heat shock. J Neurosci. $16,478-485$.

Masliah E, Mallory M, Alford M, DeTeresa R, Hansen LA, McKeel DW Jr, Morris JC. (2001). Altered expression of synaptic proteins occurs early during progression of Alzheimer's disease. Neurology. 56, 127-129. 
Mattson MP, Gary DS, Chan SL, Duan W. (2001). Perturbed endoplasmic reticulum function, synaptic apoptosis and the pathogenesis of Alzheimer's disease. Biochem Soc Symp. 67, 151-162.

Mayer MP, Bukau B. (2005). Hsp70 chaperones: Cellular functions and molecular mechanism. Cell Mol Life Sci. 62, 670-684.

Montgomery SM, Buzsáki G. (2007). Gamma oscillations dynamically couple hippocampal CA3 and CA1 regions during memory task performance. Proc Natl Acad Sci USA. 104, 14495-14500.

Moon IS, Park IS, Schenker LT, Kennedy MB, Moon JI, Jin I. (2001). Presence of both constitutive and inducible forms of heat shock protein 70 in the cerebral cortex and hippocampal synapses. Cereb. Cortex. 11, 238-248.

Morrissette DA, Parachikova A, Green KN, LaFerla FM. (2009). Relevance of transgenic mouse models to human Alzheimer disease. J Biol Chem. 284, 6033-6037.

Muchowski PJ, Wacker JL. (2005). Modulation of neurodegeneration by molecular chaperones. Nat Rev Neurosci. 6, 11-22.

Muchowski PJ. (2002). Protein misfolding, amyloid formation, and neurodegeneration: a critical role for molecular chaperones? Neuron 35, 9-12.

Mucke L, Masliah E, Yu GQ, Mallory M, Rockenstein EM, Tatsuno G, Hu K, Kholodenko D, Johnson-Wood K, McConlogue L. (2000). High-level neuronal expression of $A \beta 1-42$ in wild-type human amyloid protein precursor transgenic mice: synaptotoxicity without plaque formation. J Neurosci. 20, 4050-4058.

Näslund J, Schierhorn A, Hellman U, Lannfelt L, Roses AD, Tjernberg LO, Silberring J, Gandy SE, Winblad B, Greengard P, et al. (1994). Relative abundance of Alzheimer A beta amyloid peptide variants in Alzheimer disease and normal aging. Proc Natl Acad Sci USA. 91, 8378-8382.

Neef DW, Jaeger A M, Thiele DJ. (2011). Heat shock transcription factor 1 as a therapeutic target in neurodegenerative diseases. Nat Rev Drug Discov. 10, 930-944.

Neckers L. (2007). Heat shock protein 90: the cancer chaperone. J Biosci. 32, 517-530.

Neckers L.(2002). Hsp90 inhibitors as novel cancer chemotherapeutic agents. Trends Mol Med. 8, S55-61.

National Institute of Neurological Disorders and Stroke. (2013). "Amyotrophic Lateral Sclerosis (ALS)Fact Sheet". NIH Publication. 13-916. 
Nollen EA, and Morimoto RI. (2002). Chaperoning signaling pathways: molecular chaperones as stress-sensing 'heat shock' proteins. J Cell Sci. 115, 2809-2816.

Oakley H, Cole SL, Logan S, Maus E, Shao P, Craft J, Guillozet-Bongaarts A, Ohno M, Disterhoft J, Van Eldik L, et al. (2006). Intraneuronal beta-amyloid aggregates, neurodegeneration, and neuron loss in transgenic mice with five familial Alzheimer's disease mutations: potential factors in amyloid plaque formation. J Neurosci. 26, 1012910140 .

Oddo S, Caccamo A, Shepherd JD, Murphy MP, Golde TE, Kayed R, Metherate R, Mattson MP, Akbari Y, LaFerla FM. (2003). Triple-transgenic model of Alzheimer's disease with plaques and tangles: intracellular Abeta and synaptic dysfunction. Neuron. $39,409-421$.

Onos KD, Sukoff Rizzo SJ, Howell GR, Sasner M. (2016). Toward more predictive genetic mouse models of Alzheimer's disease. Brain Res Bull. 122, 1-11.

Oster T, Pillot T. (2010). Docosahexaenoic acid and synaptic protection in Alzheimer's disease mice. Biochim Biophys Acta. 1801, 791-798.

Pagani L, Eckert A. (2011). Amyloid-Beta Interaction with Mitochondria, Int J Alzheimers Dis. 2011, 925050.

Palop JJ, Mucke L. (2010). Amyloid-beta-induced neuronal dysfunction in Alzheimer's disease: from synapses toward neural networks. Nat Neurosci. 13, 812-818.

Parihar MS, Brewer GJ. (2010). Amyloid- $\beta$ as a modulator of synaptic plasticity. J Alzheimers Dis. 22, 741-763.

Pearl LH, Prodromou C, Workman P. (2008). The Hsp90 molecular chaperone: an open and shut case for treatment. Biochem J. 410, 439-453.

Penzes P, Cahill ME, Jones KA, VanLeeuwen JE, Woolfrey KM. (2011). Dendritic spine pathology in neuropsychiatric disorders. Nat Neurosci. 14, 285-293.

Perry VH, Newman TA, Cunningham C. (2003). The impact of systemic infection on the progression of neurodegenerative disease. Nat Rev Neurosci. 4, 103-112.

Picard D. (2008). Hsp90 interactors.

http://www.picard.ch/downloads/Hsp90interactors.pdf.

Pitt J, Thorner M, Brautigan D, Larner J, Klein WL. (2013). Protection against the synaptic targeting and toxicity of Alzheimer's-associated $A \beta$ oligomers by insulin mimetic chiro-inositols. FASEB J. 27, 199-207. 
Prahlad V., Morimoto R.I .(2009). Integrating the stress response: lessons for neurodegenerative diseases from C. elegans. Trends Cell Biol.19, 52-61.

Price PJ, Suk WA, Skeen PC, Spahn GJ, Chirigos MA. (1977). Geldanamycin inhibition of 3-methylcholanthrene-induced rat embryo cell transformation. Proc Soc Exp Biol Med. 155, 461-463.

Pringsheim T, Jette N, Frolkis A, Steeves TD. (2014). The prevalence of Parkinson's disease: a systematic review and meta-analysis. Mov Disord. 29, 1583-1590.

Pringsheim T, Wiltshire K, Day L, Dykeman J, Steeves T, Jette N. (2012). The incidence and prevalence of Huntington's disease: a systematic review and meta-analysis. Mov Disord. 27, 1083-1091.

Proisy N, Sharp SY, Boxall K, Connelly S, Roe SM, Prodromou C, Slawin AM, Pearl LH, Workman P, Moody CJ. (2006). Inhibition of Hsp90 with synthetic macrolactones: synthesis and structural and biological evaluation of ring and conformational analogs of radicicol. Chem Biol. 13, 1203-1215.

Prudencio M , Borchelt DR. (2011). Superoxide dismutase 1 encoding mutations linked to ALS adopts a spectrum of misfolded states. Mol Neurodegener. 6, 77.

Purves D, Augustine GJ, Fitzpatrick D, Katz LC, LaMantia AS, McNamara JO, and Williams SM. (2001). Neuroscience, 2nd edition, Sinauer Associates.

Putcha P, Danzer KM, Kranich LR, Scott A, Silinski M, et al. (2010) Brainpermeable small-molecule inhibitors of Hsp90 prevent alpha-synuclein oligomer formation and rescue alpha-synuclein-induced toxicity. J Pharmacol Exp Ther 332, 849-857.

Reddy PH, Mani G, Park BS, Jacques J, Murdoch G, Whetsell W Jr, Kaye J, Manczak M. (2005). Differential loss of synaptic proteins in Alzheimer's disease: implications for synaptic dysfunction. J Alzheimers Dis. 7, 103-117.

Riddle DR and Lichtenwalner RJ. (2007). Brain Aging: Models, Methods and Mechanisms. Chapter 6 Neurogenesis in the Adult and Aging Brain. CRC Press/Taylor \& Francis.

Riedel M, Goldbaum O, Schwarz L, Schmitt S, Richter-Landsberg C. (2010). 17-AAG induces cytoplasmic alpha-synuclein aggregate clearance by induction of autophagy. PLoS One 5, e8753.

Ritossa F. (1962). A new puffing pattern induced by temperature shock and DNP in Drosophila. Experientia. 18, 571-573. 
Riva L, Koeva M, Yildirim F, Pirhaji L, Dinesh D, Mazor T, Duennwald ML, Fraenkel E. (2012). Poly-glutamine expanded huntingtin dramatically alters the genome wide binding of HSF1. J. Huntingtons Dis. 1, 33-45.

Ross CA, Poirier MA. (2004). Protein aggregation and neurodegenerative disease. Nat Med. 10, S10-17.

Saito T, Matsuba Y, Mihira N, Takano J, Nilsson P, Itohara S, Iwata N, Saido TC. (2014). Single App knock-in mouse models of Alzheimer's disease. Nat Neurosci. 17, 661-663.

Sakono M, Zako T. (2010). Amyloid oligomers: formation and toxicity of Abeta oligomers. FEBS J. 277, 1348-1358

Sankari Z, Adeli H, Adeli A. (2012). Wavelet coherence model for diagnosis of Alzheimer disease. Clin EEG Neurosci. 43, 268-278.

Sano M. (2001). Radicicol and geldanamycin prevent neurotoxic effects of anti-cancer drugs on cultured embryonic sensory neurons. Neuropharmacology. 40, 947-953.

Santacruz K, Lewis J, Spires T, Paulson J, Kotilinek L, Ingelsson M, Guimaraes A, DeTure M, Ramsden M, McGowan E. (2005). Tau suppression in a neurodegenerative mouse model improves memory function. Science. 309, 476-481.

Sarge KD, Murphy SP, and Morimoto RI. (1993). Activation of heat shock gene transcription by heat shock factor 1 involves oligomerization, acquisition of DNAbinding activity, and nuclear localization and can occur in the absence of stress. Mol Cell Biol. 13, 1392-1407.

Sasaki K, Rinehart KL Jr, Slomp G, Grostic MF, Olson EC. (1970). Geldanamycin. I. Structure assignment. J Am Chem Soc. 92, 7591-7593.

Savonenko AV, Xu GM, Price DL, Borchelt DR, Markowska AL. (2003). Normal cognitive behavior in two distinct congenic lines of transgenic mice hyperexpressing mutant APPSWE. Neurobiol Dis. 12, 194-211.

Schaeffer EL, Figueiro M, Gattaz WF. (2011). Insights into Alzheimer disease pathogenesis from studies in transgenic animal models. Clinics (Sao Paulo) 66, 45-54.

Schindowski K, Bretteville A, Leroy K, Bégard S, Brion JP, Hamdane M, Buée L. (2006). Alzheimer's disease-like tau neuropathology leads to memory deficits and loss of functional synapses in a novel mutated tau transgenic mouse without any motor deficits. Am J Pathol 169, 599-616.

Schlesinger MJ. (1990). "Heat shock proteins". J. Biol. Chem. 265 (21): 12111-12114. 
Schmechel DE, Saunders AM, Strittmatter WJ, Crain BJ, Hulette CM, Joo SH, PericakVance MA, Goldgaber D, Roses AD. (1993). Increased amyloid beta-peptide deposition in cerebral cortex as a consequence of apolipoprotein E genotype in late-onset Alzheimer disease. Proc Natl Acad Sci USA. 90, 9649-9653.

Schulte TW, Akinaga S, Soga S, Sullivan W, Stensgard B, Toft D, Neckers LM. (1998). Antibiotic radicicol binds to the N-terminal domain of Hsp90 and shares important biologic activities with geldanamycin. Cell Stress Chaperones. 3, 100-108.

Schulte TW, Neckers LM. (1998). The benzoquinone ansamycin 17-allylamino-17demethoxygeldanamycin binds to Hsp90 and shares important biologic activities with geldanamycin. Cancer Chemother Pharmacol. 42, 273-279.

Segal M. (2005). Dendritic spines and long-term plasticity. Nat Rev Neurosci. 6, 277 284.

Selkoe DJ. (2008). Soluble oligomers of the amyloid $\beta$-protein impair synaptic plasticity and behavior. Behav Brain Res. 192, 106-113.

Shankar GM, Bloodgood BL, Townsend M, Walsh DM, Selkoe DJ, Sabatini BL. (2007). Natural Oligomers of the Alzheimer Amyloid- $\beta$ Protein Induce Reversible Synapse Loss by Modulating an NMDA-Type Glutamate Receptor-Dependent Signaling Pathway. J Neurosci. 27, 2866-2875.

Shankar GM and Walsh DM. (2009). Alzheimer's disease: synaptic dysfunction and A $\beta$. Mol Neurodegener. 4, 48.

Shetty AK, Hattiangady B, Rao MS, Shuai B. (2011). Deafferentation enhances neurogenesis in the young and middle aged hippocampus but not in the aged hippocampus. Hippocampus. 2011 21, 631-646.

Shiotsu Y, Neckers LM, Wortman I, An WG, Schulte TW, Soga S, Murakata C, Tamaoki T, Akinaga S. (2000). Novel oxime derivatives of radicicol induce erythroid differentiation associated with preferential G(1) phase accumulation against chronic myelogenous leukemia cells through destabilization of Bcr-Abl with Hsp90 complex. Blood. 96, 2284-2291.

Shukla V, Mishra SK, Pant HC. (2011). Oxidative Stress in Neurodegeneration. Adv Pharmacol Sci. 2011, 572634.

Sittler A, Lurz R, Lueder G, Priller J, Lehrach H, Hayer-Hartl MK, Hartl FU, Wanker EE. (2001). Geldanamycin activates a heat shock response and inhibits huntingtin aggregation in a cell culture model of Huntington's disease. Hum Mol Genet. 10, 13071315. 
Soga S, Neckers LM, Schulte TW, Shiotsu Y, Akasaka K, Narumi H, Agatsuma T, Ikuina Y, Murakata C, Tamaoki T, et al. (1999). KF25706, a novel oxime derivative of radicicol, exhibits in vivo antitumor activity via selective depletion of Hsp90 binding signaling molecules. Cancer Res. 59, 2931-2938.

Soga S, Sharma SV, Shiotsu Y, Shimizu M, Tahara H, Yamaguchi K, Ikuina Y, Murakata C, Tamaoki T, Kurebayashi J, et al. (2001). Stereospecific antitumor activity of radicicol oxime derivatives. Cancer Chemother Pharmacol. 48, 435-445.

Soga S, Shiotsu Y, Akinaga S, Sharma SV. (2003). Development of radicicol analogues. Curr Cancer Drug Targets. 3, 359-369.

Soscia SJ, Kirby JE, Washicosky KJ, Tucker SM, Ingelsson M, Hyman B, Burton MA, Goldstein LE, Duong S, Tanzi RE, et al. (2010). The Alzheimer's disease-associated amyloid beta-protein is an antimicrobial peptide. PLoS One 5, e9505.

Soto C, Estrada LD. (2008). Protein misfolding and neurodegeneration. Arch Neurol. 65, 184-189.

Spellman T, Rigotti M, Ahmari SE, Fusi S, Gogos JA, Gordon JA. (2015). Hippocampalprefrontal input supports spatial encoding in working memory. Nature 522, 309-314.

Spires-Jones TL, Mielke ML, Rozkalne A, Meyer-Luehmann M, de Calignon A, Bacskai BJ, Schenk D, Hyman BT. (2009). Passive immunotherapy rapidly increases structural plasticity in a mouse model of Alzheimer disease. Neurobiol. Dis. 33, 213-220.

Spires-Jones TL, Orne JD, Santacruz K, Pitstick R, Carlson GA, Ashe KH, Hyman BT. (2006). Region-specific dissociation of neuronal loss and neurofibrillary pathology in a mouse model of tauopathy. Am J Pathol. 168, 1598-1607.

Sreedhar AS, Kalmár E, Csermely P, Shen YF. (2004). Hsp90 isoforms: functions, expression and clinical importance. FEBS Lett. 562, 11-15.

Stetler RA, Gan Y, Zhang W, Liou AK, Gao Y, Cao G, Chen J. (2010). Heat shock proteins: cellular and molecular mechanisms in the central nervous system. Prog Neurobiol. 92, 184-211.

Strittmatter WJ, Saunders AM, Schmechel D, Pericak-Vance M, Enghild J, Salvesen GS, Roses AD. (1993). Apolipoprotein E: high-avidity binding to beta-amyloid and increased frequency of type 4 allele in late-onset familial Alzheimer disease. Proc Natl Acad Sci USA. 90, 1977-1981.

Sturchler-Pierrat C, Abramowski D, Duke M, Wiederhold KH, Mistl C, Rothacher S, Ledermann B, Bürki K, Frey P, Paganetti PA, et al. (1997). Two amyloid precursor protein transgenic mouse models with Alzheimer disease-like pathology. Proc Natl Acad Sci USA. 94, 13287-13292. 
Sun Y, Wu S, Bu G, Onifade MK, Patel SN, LaDu MJ, Fagan AM, Holtzman DM. (1998). Glial fibrillary acidic protein-apolipoprotein E (apoE) transgenic mice: astrocytespecific expression and differing biological effects of astrocyte-secreted apoE3 and apoE4 lipoproteins. J Neurosci. 18, 3261-3272.

Supko JG, Hickman RL, Grever MR, Malspeis L. (1995). Preclinical pharmacologic evaluation of geldanamycin as an antitumor agent. Cancer Chemother Pharmacol. 36, 305-315.

Sze CI, Troncoso JC, Kawas C, Mouton P, Price DL, Martin LJ. (1997). Loss of the presynaptic vesicle protein synaptophysin in hippocampus correlates with cognitive decline in Alzheimer disease. J Neuropathol Exp Neurol. 56, 933-944.

Tabaton M, Zhu X, Perry G, Smith MA, Giliberto L. (2010). Signaling effect of amyloidbeta(42) on the processing of A PPP. Experimental Neurology 221, 18-25.

Taipale M, Jarosz DF, Lindquist S. (2010). Hsp90 at the hub of protein homeostasis: emerging mechanistic insights. Nat Rev Mol Cell Biol. 11, 515-528.

Taldone T, Gillan V, Sun W, Rodina A, Patel P, Maitland K, O'Neill K, Chiosis G, Devaney E. (2010). Assay strategies for the discovery and validation of therapeutics targeting Brugia pahangi Hsp90. PLoS Negl Trop Dis. 4, e714.

Tanemura K, Murayama M, Akagi T, Hashikawa T, Tominaga T, Ichikawa M, Yamaguchi H, Takashima A. (2002). Neurodegeneration with tau accumulation in a transgenic mouse expressing V337M human tau. J Neurosci 22, 133-141.

Terry RD, Masliah E, Salmon DP, Butters N, DeTeresa R, Hill R, Hansen LA, Katzman R. (1991). Physical basis of cognitive alterations in Alzheimer's disease: synapse loss is the major correlate of cognitive impairment. Ann Neurol. 30, 572-580.

Terwel D, Lasrado R, Snauwaert J, Vandeweert E, Van Haesendonck C, Borghgraef P, Van Leuven F. (2005). Changed conformation of mutant Tau-P301L underlies the moribund tauopathy, absent in progressive, nonlethal axonopathy of Tau- $4 \mathrm{R} / 2 \mathrm{~N}$ transgenic mice. J Biol Chem. 280, 3963-3973.

The Huntington's Disease Collaborative Research Group. (1993). A novel gene containing a trinucleotide repeat that is expanded and unstable on Huntington's disease chromosomes. Cell. 72, 971-983.

Thomas M, Harrell JM, Morishima Y, Peng HM, Pratt WB, et al. (2006). Pharmacologic and genetic inhibition of hsp90-dependent trafficking reduces aggregation and promotes degradation of the expanded glutamine androgen receptor without stress protein induction. Hum Mol Genet. 15, 1876-1883. 
Tokui K, Adachi H, Waza M, Katsuno M, Minamiyama M, et al. (2009). 17-DMAG ameliorates polyglutamine-mediated motor neuron degeneration through well-preserved proteasome function in an SBMA model mouse. Hum Mol Genet. 18, 898-910.

Trinklein ND, Murray JI, Hartman SJ, Botstein D, Myers RM. (2004). The role of heat shock transcription factor 1 in the genome-wide regulation of the mammalian heat shock response. Mol. Biol. Cell 15, 1254-1261.

Tully T, Bourtchouladze R, Scott R, Tallman J. (2003). Targeting the CREB pathway for memory enhancers. Nat Rev Drug Discov. 2, 267-277.

Uttara B, Singh AV, Zamboni P, Mahajan RT. (2009). Oxidative stress and neurodegenerative diseases: a review of upstream and downstream antioxidant therapeutic options. Curr Neuropharmacol. 7, 65-74.

Verma P., Pfister JA., Mallick S., D'Mello SR. (2014). HSF1 protects neurons through a novel trimerization- and Hsp-independent mechanism. J Neurosci. 34, 1599-1612.

Walsh D, Klyubin I, Fadeeva J, Cullen WK, Anwyl R, Wolfe M, Rowan M, Selkoe D. (2002). Naturally secreted oligomers of the Alzheimer amyloid $\beta$-protein potently inhibit hippocampal long-term potentiation in vivo. Nature. 416, 535-539.

Wang C, Barluenga S, Koripelly GK, Fontaine JG, Chen R, Yu JC, Shen X, Chabala JC, Heck JV, Rubenstein A, et al. (2009). Synthesis of pochoxime prodrugs as potent Hsp90 inhibitors. Bioorganic \& medicinal chemistry letters. 19, 3836-3840.

Warrick JM, Chan HY, Gray-Board GL, Chai Y, Paulson HL, Bonini NM. (1999). Suppression of polyglutaminemediated neurodegeneration in Drosophila by the molecular chaperone Hsp70. Nat Genet. 23, 425-428.

Waza M, Adachi H, Katsuno M, Minamiyama M, Sang C, Tanaka F, Inukai A, Doyu M, Sobue G. (2005). 17-AAG, an Hsp90 inhibitor, ameliorates polyglutamine-mediated motor neurondegeneration. Nat Med. 11, 1088-1095.

Wei W, Nguyen LN, Kessels HW, Hagiwara H, Sisodia S, Malinow R. (2010). Amyloid beta from axons and dendrites reduces local spine number and plasticity. Nat Neurosci. 13, 190-196.

Welch WJ, Kang HS, Beckmann RP, and Mizzen LA. (1991). Response of mammalian cells to metabolic stress; changes in cell physiology and structure/function of stress proteins. Current topics in microbiology and immunology. 167, 31-55.

Westerheide SD, Morimoto RI. (2005). Heat shock response modulators as therapeutic tools for diseases of protein conformation. J Biol Chem. 280, 33097-33100. 
Westerman MA, Cooper-Blacketer D, Mariash A, Kotilinek L, Kawarabayashi T, Younkin LH, Carlson GA, Younkin SG, Ashe KH. (2002). The relationship between A $\beta$ and memory in the Tg2576 mouse model of Alzheimer's disease. J Neurosci. 22,18581867.

Whitesell L, Lindquist SL. (2005). Hsp90 and the chaperoning of cancer. Nat Rev Cancer $5,761-772$.

Wilson RS, Segawa E, Boyle, PA, Anagnos SE, Hizel LP, Bennett DA. (2012). The natural history of cognitive decline in Alzheimer's disease. Psychol Aging. 27, 10081017.

Yoon YJ, Kim JA, Shin KD, Shin DS, Han YM, Lee YJ, Lee JS, Kwon BM, Han DC. (2011). KRIBB11 inhibits Hsp70 synthesis through inhibition of heat shock factor 1 function by impairing the recruitment of positive transcription elongation factor $b$ to the hsp70 promoter. J. Biol. Chem. 286, 1737-1747.

Young JC, Moarefi I, Hartl FU. (2001). Hsp90: a specialized but essential protein-folding tool. J Cell Biol. 154, 267-273.

Yu JY, Frank LM. (2015). Hippocampal-cortical interaction in decision making. Neurobiol Learn Mem. 117, 34-41.

Zhang W, Han SW, McKeel DW, Goate A, Wu JY. (1998). Interaction of presenilins with the filamin family of actin-binding proteins. J Neurosci. 18, 914-922.

Zhang Y, Frejtag W, Dai R, Mivechi NF. (2001). Heat shock factor-4 (HSF-4a) is a repressor of HSF-1 mediated transcription. J Cell Biochem. 82, 692-703.

Zhu H, Woolfenden S, Bronson RT, Jaffer ZM, Barluenga S, Winssinger N, Rubenstein AE, Chen R, Charest A. (2010). The novel Hsp90 inhibitor NXD30001 induces tumor regression in a genetically engineered mouse model of glioblastoma multiforme. Mol Cancer Ther. 9, 2618-2626.

Zou J, Guo Y, Guettouche T, Smith DF, Voellmy R. (1998). Repression of heat shock transcription factor HSF1activation by Hsp90 (Hsp90 complex) that forms a stresssensitive complex with HSF1". Cell. 94, 471-480. 


\section{VITA}

Bin Wang was born in Jiangsu Province of P.R.China in 1985. During 2003-2007, he attended Nanjing Medical University in Nanjing, Jiangsu in the major of Pharmacy. He attained his bachelor's degree in June 2007. During 2007-2010, he attended the graduate school in school of pharmacy at Nanjing Medical University in the major of pharmacology. He attained his master's degree in June 2010. During the graduate study at Nanjing Medical University, he was exchanged to Dr. Francesca-Fang Liao's lab as a visiting scholar for one and a half years. In January 2011, he enrolled in the doctoral track of the Integrated Program in Biomedical Sciences at the University of Tennessee Health Science Center. He joined the laboratory of Dr. Francesca-Fang Liao and has been conducted research in aging-related diseases.

Mr. Wang's publications include:

1. Wang B, Liu Y, Huang, L, Chen J, Li JJ, Wang R, Kim E, Chen Y, Justicia C, Sakata K, Chen H, Planas A, Ostrom RS, Li W, Yang G, McDonald MP, Chen R, Heck DH, Liao FF. (2016). A CNS-permeable Hsp90 inhibitor rescues synaptic dysfunction and memory loss in APP-overexpressing Alzheimer's mouse model via an HSF1-mediated mechanism. Mol Psychiatry. In press.

2. Kim E, Wang B, Sastry N, Nelson P, Cai HB, Liao FF. (2016). NEDD4-mediated HSF1 degradation underlies $\alpha$-synucleinopathy. Hum Mol Genet. 25, 211-222.

3. Chen YP, Zhang DQ, Liao Z, Wang B, Gong S, Wang C, Zhang MZ, Wang GH, Cai H, Liao FF, Xu JP. (2015). Anti-oxidant polydatin (piceid) protects against substantia nigral motor degeneration in multiple rodent models of Parkinson's disease. Mol Neurodegener. 2015. 10, doi: 10.1186/1750-1326-10-4.

4. Chen Y*, Wang B* (*Co-first author), Liu D, Li JJ, Xue Y, Sakata K, Zhu LQ, Heldt SA, Xu H, Liao FF. (2014). Hsp90 chaperone inhibitor 17-AAG attenuates A $\beta$-induced synaptic toxicity and memory impairment. J Neurosci. 34, 2464-2470.

5. Kwak YD, Wang B, Li JJ, Wang R, Deng Q, Diao S, Chen Y, Xu R, Masliah E, Xu H, Sung JJ, Liao FF. (2012). Upregulation of the E3 ligase NEDD4-1 by oxidative stress degrades IGF-1 receptor protein in neurodegeneration. J Neurosci. 32, 1097110981.

6. Luo CX, Jin X, Cao CC, Zhu MM, Wang B, Chang L, Zhou QG, Wu HY, Zhu DY. (2010). Bidirectional regulation of neurogenesis by neuronal nitric oxide synthase derived from neurons and neural stem cells. Stem Cell. 28, 2041-2052.

7. Kwak YD, Wang B, Pan W, Xu H, Jiang X, Liao FF. (2010). Functional interaction of phosphatase and tensin homologue (PTEN) with the E3 ligase NEDD4-1 during neuronal response to zinc. Journal of Biological Chemistry. 285, 9847-9857. 
8. Li WL, Cai HH, Wang B, Chen L, Zhou QG, Luo CX, Liu N, Ding XS, Zhu DY. (2009). Chronic Fluoxetine Treatment Improves Ischemia-Induced Spatial

Cognitive Deficits Through Increasing Hippocampal Neurogenesis After Stroke, Journal of Neuroscience Research. 87, 112-122.

9. Luo CX, Zhu XJ, Zhou QG, Wang B, Wang W, Cai HH, Sun YJ, Hu M, Jiang J, Hua Y, Han X, Zhu DY.(2007). Reduced neuronal nitric oxide synthase is involved in ischemia-induced hippocampal neurogenesis by up-regulating inducible nitric oxide synthase expression, Journal of Neurochemistry.103, 1872-1882.

10. Zhou QG, Hu Y, Hua Y, Hu M, Luo CX, Han X, Zhu XJ, Wang B, Xu JS, Zhu DY. (2007). Neuronal nitric oxide synthase contributes to chronic stress-induced depression by suppressing hippocampal neurogenesis, Journal of Neurochemistry. $103,1843-1854$. 